\title{
INFINITE GRASSMANNIANS AND MODULI SPACES OF $G$-BUNDLES
}

\author{
SHRAWAN KUMAR
}

\section{Introduction.}

These are notes for my eight lectures given at the C.I.M.E. session on "Vector bundles on curves. New directions" held at Cetraro (Italy) in June 1995. The work presented here was done in collaboration with M.S. Narasimhan and A. Ramanathan and appeared in $[\mathrm{KNR}]$. These notes differ from $[\mathrm{KNR}]$ in that we have added three appendices (A)-(C) containing basic definitions and results (we need) on ind-varieties, Kac-Moody Lie algebras, the associated groups and their flag varieties. We also have modified the proof (given in $\S 7$ ) of basic extension result (Proposition 6.5), and we hope that it is more transparent than the one given in $[\mathrm{KNR}, \S 7]$. We now describe the main result of this note.

Let $C$ be a smooth projective irreducible algebraic curve over $\mathbb{C}$ of any genus and $G$ a connected simply-connected simple affine algebraic group over $\mathbb{C}$. In this note we elucidate the relationship between

(1) the space of vacua ("conformal blocks") defined in Conformal Field Theory, using an integrable highest weight representation of the affine Kac-Moody algebra associated to $G$ and

(2) the space of regular sections ("generalised theta functions") of a line bundle on the moduli space $\mathfrak{M}$ of semistable principal $G$-bundles on $C$.

Fix a point $p$ in $C$ and let $\hat{\mathcal{O}}_{p}$ (resp. $\hat{k}_{p}$ ) be the completion of the local ring $\mathcal{O}_{p}$ of $C$ at $p$ (resp. the quotient field of $\hat{\mathcal{O}}_{p}$ ). Let $\mathcal{G}:=G\left(\hat{k}_{p}\right)$ (the $\hat{k}_{p}$-rational points of the algebraic group $G$ ) be the loop group of $G$ and let $\mathcal{P}:=G\left(\hat{\mathcal{O}}_{p}\right)$ be the standard maximal parahoric subgroup of $\mathcal{G}$. Then the generalised flag variety $X:=\mathcal{G} / \mathcal{P}$ is an inductive limit of projective varieties, in fact of generalised Schubert varieties. One has a basic homogeneous line bundle $\mathfrak{L}\left(\chi_{o}\right)$ on $X$ (cf. $\S$ C.6), and the Picard group $\operatorname{Pic}(X)$ is isomorphic to $\mathbb{Z}$ which is generated by $\mathfrak{L}\left(\chi_{o}\right)$ (Proposition C.13). There is a central extension $\tilde{\mathcal{G}}$ of $\mathcal{G}$ by the multiplicative group $\mathbb{C}^{*}$ (cf. $\S \mathrm{C} .4$ ), which acts on the line bundle $\mathfrak{L}\left(\chi_{o}\right)$. By an analogue of the Borel-Weil theorem proved in the Kac-Moody setting by Kumar (and also by Mathieu), the space $H^{0}\left(X, \mathfrak{L}\left(d \chi_{o}\right)\right.$ ) of the regular sections of the line bundle $\mathfrak{L}\left(d \chi_{o}\right):=\mathfrak{L}\left(\chi_{o}\right)^{\otimes d}$ (for any $d \geq 0$ ) is canonically isomorphic with the full vector space dual $L(\mathbb{C}, d)^{*}$ of the integrable highest weight (irreducible) module $L(\mathbb{C}, d)$ (with central charge $d$ ) of the affine Kac-Moody Lie algebra $\tilde{\mathfrak{g}}$ (cf. §A.2).

Using the fact that any principal $G$-bundle on $C \backslash p$ is trivial (Proposition 1.3), one sees easily that the set of isomorphism classes of principal $G$-bundles on $C$ 
is in bijective correspondence with the double coset space $\Gamma \backslash \mathcal{G} / \mathcal{P}$, where $\Gamma:=$ $\operatorname{Mor}(C \backslash p, G)$ is the subgroup of $\mathcal{G}$ consisting of all the algebraic morphisms $C \backslash p \rightarrow$ $G$. Moreover, $X$ parametrizes an algebraic family $\mathcal{U}$ of principal $G$-bundles on $C$ (cf. Proposition 2.8). As an interesting byproduct of this parametrization and rationality of the generalised Schubert varieties $X_{\mathfrak{w}}$, we obtain that the moduli space $\mathfrak{M}$ of semistable principal $G$-bundles on $C$ is a unirational variety (cf. Corollary 6.3). Now, given a finite dimensional representation $V$ of $G$, let $\mathcal{U}(V)$ be the family of associated vector bundles on $C$ parametrized by $X$. We have then the determinant line bundle $\operatorname{Det}(\mathcal{U}(V))$ on $X$, defined as the dual of the determinant of the cohomology of the family $\mathcal{U}(V)$ of vector bundles on $C$ (cf. §3.7). As we mentioned above, Pic $(X)$ is freely generated by the homogeneous line bundle $\mathfrak{L}\left(\chi_{o}\right)$ on $X$, in particular, there exists a unique integer $m_{V}$ (depending on the choice of the representation $V$ ) such that $\operatorname{Det}(\mathcal{U}(V)) \simeq \mathfrak{L}\left(m_{V} \chi_{o}\right)$. We determine this number explicitly in Theorem (5.4), the proof of which makes use of RiemannRoch theorem. It is shown that the number $m_{V}$ coincides with the Dynkin index of the representation $V$. For example, if we take $V$ to be the adjoint representation of $G$, then $m_{V}=2 \times$ dual Coxeter number of $G$ (cf. Lemma 5.2 and Remark 5.3). The number $m_{V}$ is also expressed in terms of the induced map at the third homotopy group level $\pi_{3}(G) \rightarrow \pi_{3}(S L(V))$ (cf. Corollary 5.6).

The action of $\Gamma$ on $X$ via left multiplication lifts to an action on the line bundle $\mathfrak{L}\left(m_{V} \chi_{o}\right)$ (cf. $\left.\S 2.7\right)$. Suggested by Conformal Field Theory, we consider the space $H^{0}\left(X, \mathfrak{L}\left(d m_{V} \chi_{o}\right)\right)^{\Gamma}$ of $\Gamma$-invariant regular sections of the line bundle $\mathfrak{L}\left(d m_{V} \chi_{o}\right)$ (for any $d \geq 0$ ). This space of invariants is called the space of vacua. More precisely, in Conformal Field Theory, the space of vacua is defined to be the space of invariants of the Lie algebra $\mathfrak{g} \otimes R$ in $L(\mathbb{C}, d)^{*}$, where $R$ is the ring of regular functions on the affine curve $C \backslash p$ and $\mathfrak{g}$ is the Lie algebra of the group $G$. We have (by Proposition 6.7) $\left[L\left(\mathbb{C}, d m_{V}\right)^{*}\right]^{\Gamma}=\left[L\left(\mathbb{C}, d m_{V}\right)^{*}\right]^{\mathfrak{g} \otimes R}$ and, as already mentioned above, $H^{0}\left(X, \mathfrak{L}\left(d m_{V} \chi_{o}\right)\right) \simeq L\left(\mathbb{C}, d m_{V}\right)^{*}$. The main result of this note (Theorem 6.6) asserts that (for any $d \geq 0$ ) the space $H^{0}\left(\mathfrak{M}, \Theta(V)^{\otimes d}\right)$ of the regular sections of the $d$-th power of the $\Theta$-bundle $\Theta(V)$ (cf. $\S 3.7$ ) on the moduli space $\mathfrak{M}$ is isomorphic with the space of vacua $\left[L\left(\mathbb{C}, d m_{V}\right)^{*}\right]^{\Gamma}=\left[L\left(\mathbb{C}, d m_{V}\right)^{*}\right]^{\mathfrak{g} \otimes R}$. This is the connection, alluded to in the beginning of the introduction, between the space of vacua and the space of generalised theta functions. This result has also independently been obtained by Faltings [Fa] and in the case of $G=S L_{N}$ by Beauville-Laszlo [BL], both by different methods .

We make crucial use of a 'descent' lemma (cf. Proposition 4.1), and an extension result (cf. Proposition 6.5) in the proof of Theorem (6.6). The proof of Proposition (6.5) is given in $\S 7$, and relies on the explicit GIT construction of the moduli space of vector bundles.

Our Theorem (6.6) can be generalised to the situation where the curve $C$ has $n$ marked points $\left\{p_{1}, \ldots, p_{n}\right\}$ together with finite dimensional $G$-modules $\left\{V_{1}, \ldots, V_{n}\right\}$ attached to them respectively, by bringing in moduli space of parabolic $G$-bundles on $C$.

A purely algebro-geometric study (which does not use loop groups) of generalised theta fuctions on the moduli space of (parabolic) rank two torsion-free sheaves on a nodal curve is made by Narasimhan-Ramadas [NRa]. A factorization theorem and a vanishing theorem for the theta line bundle are proved there. In addition, several 
Geemen, E. Previato, A. Szenes, M. Thaddeus, R. Wentworth, D. Zagier, .. ) and physicists have studied the space of generalised theta functions (from different view points) in the case when $G=S L(2)$, in the last few years.

The organization of the note is as follows:

Apart from introducing some notation in $\S 1$, we realize the affine flag variety $X$ as a parameter set for $G$-bundles. In section (2) we prove that $X$ supports an algebraic family of G-bundles on the curve $C$ (cf. Proposition 2.8). We also realize the group $\Gamma$ as an ind-group, calculate its Lie algebra, and prove its splitting in this section. Section (3) is devoted to recalling some basic definitions and results on the moduli space of semistable $G$-bundles, including the definition of the determinant line bundle and the $\Theta$-bundle on the moduli space. We prove a curious result (cf. Proposition 4.1) on algebraic descent in $\S 4$. Section (5) is devoted to identifying the determinant line bundle on $X$ with a suitable power of the basic homogeneous line bundle on $X$. Section (6) contains the statement and the proof of the main result (Theorem 6.6). Finally in Section (7) we prove the basic extension result (Proposition 6.5), using Geometric Invariant Theory. Appendix (A) is devoted to recalling the definition of affine Kac-Moody Lie algebras and its representations. Appendix (B) is an introduction to ind-varieties and ind-groups. Finally in appendix (C), we recall the basic theory of affine Kac-Moody groups and their flag varieties.

\section{Affine flag variety as parameter set for $G$-bundles.}

(1.1) Notation. Throughout the note $k$ denotes an algebraically closed field of char. 0. By a scheme we will mean a scheme over $k$. Let us fix a smooth irreducible projective curve $C$ over $k$, and a point $p \in C$. Let $C^{*}$ denote the open set $C \backslash p$. We also fix an affine algebraic connected simply-connected simple group $G$ over $k$.

For any $k$-algebra $A$, by $G(A)$ we mean the $A$-rational points of the algebraic group $G$. We fix the following notation to be used throughout the note:

$$
\begin{aligned}
& \mathcal{G}=\mathcal{G}_{\mathfrak{T}}=G\left(\hat{k}_{p}\right), \\
& \mathcal{P}=\mathcal{P}_{\mathfrak{T}}=G\left(\hat{\mathcal{O}}_{p}\right), \text { and } \\
& \Gamma=\Gamma_{\mathfrak{T}}=G\left(k\left[C^{*}\right]\right),
\end{aligned}
$$

where $\hat{\mathcal{O}}_{p}$ is the completion of the local ring $\mathcal{O}_{p}$ of $C$ at $p, \hat{k}_{p}$ is the quotient field of $\hat{\mathcal{O}}_{p}, k\left[C^{*}\right]$ is the ring of regular functions on the affine curve $C^{*}$ (which can canonically be viewed as a subring of $\left.\hat{k}_{p}\right)$, and $\mathfrak{T}$ is the triple $(G, C, p)$.

We recall the following

(1.2) Definition. Let $H$ be any (not necessarily reductive) affine algebraic group over $k$. By a principal $H$-bundle (for short $H$-bundle) on an algebraic variety $X$, we mean an algebraic variety $E$ on which $H$ acts algebraically from the right and a $H$-equivariant morphism $\pi: E \rightarrow X$ (where $H$ acts trivially on $X$ ), such that $\pi$ is isotrivial (i.e. locally trivial in the étale topology).

Let $H$ act algebraically on a quasi-projective variety $F$ from the left. We can then form the associated bundle with fiber $F$, denoted by $E(F)$. Recall that $E(F)$ is the quotient of $E \times F$ under the $H$-action given by $g(e, f)=\left(e g^{-1}, g f\right)$, for $g \in H$, $e \in E$ and $f \in F$. 
definition, a $K$-bundle $E_{K}$ such that $E_{K}(H) \approx E$, where $K$ acts on $H$ by left multiplication. Reduction of structure group to $K$ can canonically be thought of as a section of the associated bundle $E(H / K) \rightarrow X$.

Let $\mathcal{X}=\mathcal{X}(H, C)$ denote the set of isomorphism classes of $H$-bundles on the base $C$, and $\mathcal{X}_{0}=\mathcal{X}_{0}(\mathfrak{T}) \subset \mathcal{X}$ denote the subset consisting of those $H$-bundles on $C$ which are algebraically trivial restricted to $C^{*}$. We recall the following proposition essentially due to Harder $\left[\mathrm{H}_{1}\right.$, Satz 3.3 and the remark following it].

(1.3) Proposition. Let $H$ be a connected reductive algebraic group over $k$. Then the structure group of a $H$-bundle on a smooth affine curve $Y$ can be reduced to the connected component $Z^{0}(H)$ of the centre $Z(H)$ of $H$.

In particular, if $H$ as above is semi-simple, then any $H$-bundle on $Y$ is trivial.

The following map is of basic importance for us in this note. This provides a bridge between the moduli space of $G$-bundles and the affine (Kac-Moody) flag variety, where $G$ is as in $\S 1.1$.

(1.4) Definition (of the map $\varphi: \mathcal{G} \rightarrow \mathcal{X}_{0}$ ). Consider the canonical morphisms $i_{1}: \operatorname{Spec}\left(\hat{\mathcal{O}}_{p}\right) \rightarrow C$ and $i_{2}: C^{*} \hookrightarrow C$. The morphisms $i_{1}$ and $i_{2}$ together provide a flat cover of $C$. Let us take the trivial $G$-bundle on both the $\operatorname{schemes} \operatorname{Spec}\left(\hat{\mathcal{O}}_{p}\right)$ and $C^{*}$. The fiber product

$$
F:=\operatorname{Spec}\left(\hat{\mathcal{O}}_{p}\right) \underset{C}{\times} C^{*}
$$

of $i_{1}$ and $i_{2}$ can canonically be identified with $\operatorname{Spec}\left(\hat{k}_{p}\right)$. This identification $F \simeq$ $\operatorname{Spec}\left(\hat{k}_{p}\right)$ is induced from the natural morphisms

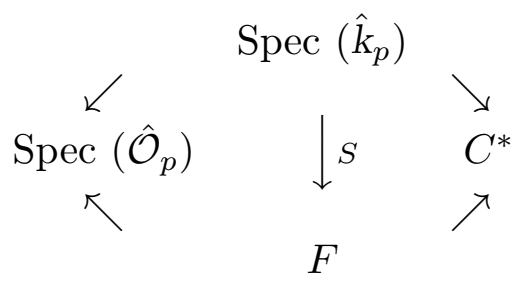

By a "glueing" lemma of Grothendieck [Mi, Part I, Theorem 2.23, p.19], to give a $G$-bundle on $C$, it suffices to give an automorphism of the trivial $G$-bundle on $\operatorname{Spec}\left(\hat{k}_{p}\right)$, i.e., to give an element of $\mathcal{G}:=G\left(\hat{k}_{p}\right)$. (Observe that since we have a flat cover of $C$ by only two schemes, the cocycle condition is vacuously satisfied.) This is, by definition, the map $\varphi: \mathcal{G} \rightarrow \mathcal{X}_{0}$.

(1.5) Proposition. The map $\varphi$ (defined above) factors through the double coset space to give a bijective map (denoted by)

$$
\bar{\varphi}: \Gamma \backslash \mathcal{G} / \mathcal{P} \rightarrow \mathcal{X}_{0}
$$

(Observe that, by Proposition (1.3), $\mathcal{X}_{0}=\mathcal{X}$ since $G$ is assumed to be connected and semi-simple.)

Proof. From the above construction, it is clear that for $g, g^{\prime} \in \mathcal{G}, \varphi(g)$ is isomor- 
isomorphisms :

$$
\begin{aligned}
\operatorname{Spec}\left(\widehat{\mathcal{O}}_{p}\right) \times G \underset{\theta_{1}}{\sim} \operatorname{Spec}\left(\widehat{\mathcal{O}}_{p}\right) \times G \\
\searrow \\
\qquad \operatorname{Spec}\left(\widehat{\mathcal{O}}_{p}\right)
\end{aligned}
$$

and

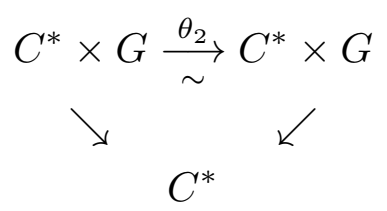

such that the following diagram is commutative:

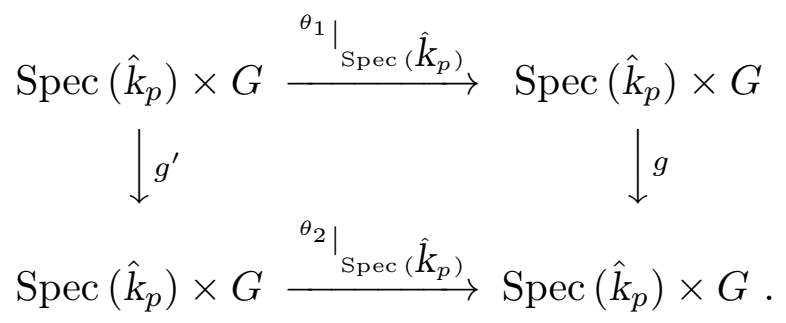

Any $G$-bundle isomorphism $\theta_{1}$ (resp. $\theta_{2}$ ) as above is given by an element $h \in \mathcal{P}$ (resp. $\gamma \in \Gamma$ ). In particular, from the commutativity of the above diagram $(*)$, $\varphi(g) \approx \varphi\left(g^{\prime}\right)$ if and only if there exists $h \in \mathcal{P}$ and $\gamma \in \Gamma$ such that $g h=\gamma g^{\prime}$, i.e., $\gamma^{-1} g h=g^{\prime}$. This shows that the map $\varphi$ factors through $\Gamma \backslash \mathcal{G} / \mathcal{P}$ to give an injective map $\bar{\varphi}$. The surjectivity of $\bar{\varphi}$ follows immediately from the definition of $\mathcal{X}_{0}$, and the fact that any $G$-bundle on $\operatorname{Spec}\left(\widehat{\mathcal{O}}_{p}\right)$ is trivial.

(1.6) Remark. $\mathcal{G} / \mathcal{P}$ should be thought of as a parameter space for $G$-bundles $E$ together with a trivialization of $E_{\left.\right|_{C^{*}}}$ (cf. Proposition 2.8).

\section{Affine flag variety parametrizing an algebraic family and realizing $\Gamma$ as an ind-group.}

Recall the definition of the group $\Gamma \subset \mathcal{G}$ from $\S 1.1$.

(2.1) Lemma. The group $\Gamma$ is an ind-group.

Proof. ${ }^{1}$ Embed $G \subset S L_{N} \hookrightarrow M_{N}$, where $M_{N}=M_{N}(k)$ is the space of $N \times$ $N$ matrices over $k$. Take a $k$-basis $\left\{f_{1}, f_{2}, f_{3}, \cdots\right\}$ of $k\left[C^{*}\right]$ (the ring of regular functions) such that $\operatorname{ord}_{p} f_{n} \leq \operatorname{ord}_{p} f_{n+1}$ for any $n \geq 1$, where $\operatorname{ord}_{p} f_{n}$ denotes the order of the pole of $f_{n}$ at $p$. There is an injective map $i: \Gamma \hookrightarrow \operatorname{Mor}\left(C^{*}, M_{N}\right)$, where Mor denotes the set of all the morphisms. The set Mor $\left(C^{*}, M_{N}\right)$ has a filtration Mor $_{0} \subseteq \ldots \subseteq$ Mor $_{n} \subseteq \ldots$, where Mor $_{n}$ is the (finite dimensional) vector space of all those morphisms $\theta: C^{*} \rightarrow M_{N}$ such that all its matrix entries have poles of order 
$\leq n$. Set $\Gamma_{n}=i^{-1}\left(\operatorname{Mor}_{n}\right)$. Any $\theta=\left(\theta_{i, j}\right) \in \operatorname{Mor}_{n}$ can be written as $\theta_{i, j}=\sum_{k=1}^{k(n)} z_{i, j}^{k} f_{k}$ (for some $k(n))$. We take $\left(z_{i, j}^{k}\right)$ as the coordinates on Mor $_{n}$. It is easy to see that $\Gamma_{n} \hookrightarrow \operatorname{Mor}_{n}$ is given by the vanishing of some polynomials in $\left(z_{i, j}^{k}\right)$, in particular, $\Gamma_{n}$ is a closed subvariety of the affine space $\mathrm{Mor}_{n}$. (We put the reduced structure on $\Gamma_{n}$.) This gives rise to the ind-variety structure on $\Gamma$ as a closed ind-subvariety of Mor $\left(C^{*}, M_{N}\right)$. It is easy to see (from the definition of the ind-variety structure on $\Gamma$ ) that $\Gamma$ in fact is an ind-group. Moreover, this ind-variety structure on $\Gamma$ does not depend upon the particular choice of the embedding $G \hookrightarrow S L_{N}$.

The following lemma determines the Lie algebra of the ind-group $\Gamma$.

(2.2) Lemma. The Lie algebra Lie $\Gamma$ is isomorphic with $\mathfrak{g} \otimes_{k} k\left[C^{*}\right]$, where $\mathfrak{g}:=$ Lie $G$ and the bracket in $\mathfrak{g} \otimes k\left[C^{*}\right]$ is defined as $[X \otimes p, Y \otimes q]=[X, Y] \otimes p q$, for $X, Y \in \mathfrak{g}$ and $p, q \in k\left[C^{*}\right]$. The isomorphism Lie $\Gamma \simeq \mathfrak{g} \otimes k\left[C^{*}\right]$ is obtained by considering the differential of the evaluation map at each point of $C^{*}$.

Proof. Choose an embedding $G \hookrightarrow S L_{N} \subset M_{N}$ as in the proof of Lemma (2.1). This gives rise to a closed immersion $i: \Gamma \hookrightarrow$ Mor $\left(C^{*}, M_{N}\right)$. In particular, it induces an injective map $d i: T_{e}(\Gamma)=\operatorname{Lie} \Gamma \hookrightarrow T_{I}$ (Mor) $\simeq$ Mor at the Zariski tangent space level (where $I$ is the identity matrix and Mor $=\operatorname{Mor}\left(C^{*}, M_{N}\right)$ ). We claim that $d i$ is a Lie algebra homomorphism, if we endow Mor $\simeq M_{N}(R)$ with the standard Lie algebra structure, where $R:=k\left[C^{*}\right]$ and $M_{N}(R)$ is the space of $N \times N$ matrices over $R$. To prove this, consider the following commutative diagram (for any fixed $\left.x \in C^{*}\right)$ :

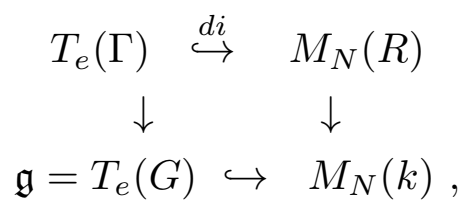

where the vertical maps are induced by the evaluation map $e_{x}: R \rightarrow k$ given by $p \mapsto p(x)$. Since the bottom horizontal map is a Lie algebra homomorphism, and so are the vertical maps, we obtain that $d i$ itself is a Lie algebra homomorphism. It is further clear, from the above commutative diagram, that the image of $d i$ is contained in $\mathfrak{g} \otimes R$, where $\mathfrak{g}$ is identified with its image in $M_{N}(k)$.

Next, we prove that the image of $d i$ contains at least the set $\mathfrak{g} \otimes R$ :

Fix any vector $X \in \mathfrak{g} \subset M_{N}(k)$ such that $X$ is a nilpotent matrix and take $p \in R$, and define a morphism $\mathbb{A}^{1} \rightarrow \Gamma$ by $z \mapsto \exp (z X \otimes p)$. (Since $X$ is nilpotent, the image is indeed contained in $\Gamma$.) It is easy to see that the image of the induced map (at the tangent space level at 0 ) is precisely the space $k(X \otimes p)$. But since the nilpotent matrices $X \in \mathfrak{g}$ span $\mathfrak{g}$, the assertion follows. This completes the proof of the lemma.

We prove the following curious lemma.

(2.3) Lemma. Let $X$ be a connected variety over $\mathbb{C}$. Then any regular map $X \rightarrow$ $\mathbb{C}^{*}$, which is null-homotopic in the topological category, is a constant.

(Observe that if the singular cohomology $H^{1}(X, \mathbb{Z})=0$, then any continuous 
Proof. Assume, if possible, that there exists a null-homotopic non-constant regular map $\lambda: X \rightarrow \mathbb{C}^{*}$. Since $\lambda$ is algebraic, there exists a number $N>0$ such that the number of irreducible components of $\lambda^{-1}(z) \leq N$, for all $z \in \mathbb{C}^{*}$. Now we consider the $N^{\prime}$-sheeted covering $\pi_{N^{\prime}}: \mathbb{C}^{*} \rightarrow \mathbb{C}^{*}\left(z \mapsto z^{N^{\prime}}\right)$, for any $N^{\prime}>N$. Since $\lambda$ is null-homotopic, there exists a (regular) lift $\tilde{\lambda}: X \rightarrow \mathbb{C}^{*}$ (cf. [Se ${ }_{1}$, Proposition 20]), making the following diagram commutative:

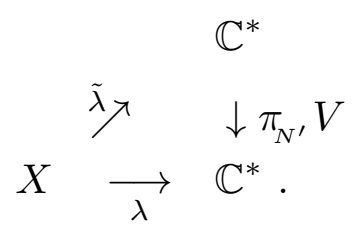

Since $\tilde{\lambda}$ is regular and non-constant, by Chevalley's theorem, Im $\tilde{\lambda}$ (being a constructible set) misses only finitely many points of $\mathbb{C}^{*}$. In particular, there exists a $z_{o} \in \mathbb{C}^{*}$ (in fact a Zariski open set of points) such that $\pi_{N^{\prime}}^{-1}\left(z_{o}\right) \subset \operatorname{Im} \tilde{\lambda}$. But then the number of irreducible components of $\lambda^{-1}\left(z_{o}\right)=\tilde{\lambda}^{-1}\left(\pi_{N^{\prime}}^{-1}\right)\left(z_{o}\right) \geq N^{\prime}>N$, a contradiction to the choice of $N$. This proves the lemma.

We will use the following corollary in the proof of assertion (c) contained in the proof of Theorem (6.6)

(2.4) Corollary. Take the base field $k=\mathbb{C}$. There does not exist any non-constant regular map $\lambda: \Gamma \rightarrow \mathbb{C}^{*}$.

Proof. The group $\Gamma$ is connected and simply-connected, in particular, $H^{1}(\Gamma, \mathbb{Z})=0$, where $H^{*}(\Gamma, \mathbb{Z})$ denotes the singular cohomology of the topological space $\Gamma$. (In fact, it is very likely that the space $\Gamma$ is homotopically equivalent to the corresponding space $\Gamma_{\text {top }}$ consisting of all the continuous maps $C^{*} \rightarrow G$ under the compact-open topology. This of course will give the connected and simple-connectedness of $\Gamma$, by using a result of Thom [GK, Theorem 5.10]. A student of mine R. Hammack is working to write a proof of this homotopy equivalence by using some ideas similar to [PS, Proof of Proposition 8.11.6], albeit in the algebraic category, together with a variant of a result of Hurtubise [Hur, Theorem 1.1].) This gives that the map $\lambda$ is null-homotopic. By using the above lemma (2.3), $\lambda$ is constant on each connected component of $\Gamma_{n}$ (for any $n \geq 0$ ) and hence $\lambda$ itself is constant.

Recall from Proposition (C.12) that $X=X_{\text {rep }}=X_{\text {lat }}$ is a projective ind-variety. (2.5) Lemma. The left multiplication of $\Gamma$ on $X$ is a morphism $\varphi: \Gamma \times X \rightarrow X$. Proof. We will consider the $X_{\text {lat }}$ description of $X$ (cf. $\S$ C.9). It is clear that $\varphi\left(\Gamma_{n} \times \hat{X}_{m}\right) \subseteq \hat{X}_{k(n, m)}$, for some $k(n, m)$. Now from the explicit description of the variety structures (on $\Gamma$ and $X_{\text {lat }}$ ), it is easy to check that $\varphi_{n, m}:=\varphi_{\mid \Gamma_{n} \times \hat{X}_{m}}$ is a morphism.

This proves the lemma.

Restrict the central extension (1) of $\S($ C.4) to get a central extension

$$
1 \rightarrow \mathbb{C}^{*} \underset{i}{\rightarrow} \tilde{\Gamma} \underset{\pi}{\rightarrow} \Gamma \rightarrow 1
$$


(2.6) Splitting of the central extension over $\Gamma$ ( $S L_{N}$ case). The basic reference for this subsection is [PS, §7.7]. We first consider the case of $G=S L_{N}$ and follow the same notation as in $\S\left(\right.$ C.7). In particular, $\mathcal{G}^{o}:=S L_{N}(\mathbb{C}((t))), \mathcal{P}^{o}=$ $S L_{N}(\mathbb{C}[[t]]), X^{o}=\mathcal{G}^{o} / \mathcal{P}^{o}, V=\mathbb{C}^{N}, V((t))=V \otimes_{\mathbb{C}} \mathbb{C}((t))$, and $L_{o}=V \otimes_{\mathbb{C}} \mathbb{C}[[t]]$. Let $G L(W)$ denote the group of $\mathbb{C}$-linear isomorphisms of a vector space $W$.

Define the subgroup $\mathcal{H}$ of $\mathcal{G}^{o} \times G L\left(L_{o}\right)$ by

$$
\mathcal{H}=\left\{(g, E) \in \mathcal{G}^{o} \times G L\left(L_{o}\right): g^{+}-E: L_{o} \rightarrow L_{o} \text { has finite rank }\right\}
$$

where $g=\left(\begin{array}{cc}g^{+} & * \\ * & *\end{array}\right)$ with respect to the decomposition

$$
V((t))=L_{o} \oplus\left(V \underset{\mathbb{C}}{\otimes} t^{-1} \mathbb{C}\left[t^{-1}\right]\right) .
$$

Let $\mathcal{N} \subset \mathcal{H}$ be the normal subgroup defined as $\mathcal{N}=\{(1, E) \in \mathcal{H}: \operatorname{det} E=1\}$. (Observe that since $I-E: L_{o} \rightarrow L_{o}$ has finite rank, i.e., has finite dimensional image, the determinant of $E$ is well defined.)

It is not difficult to see that the projection on the first factor gives rise to a central extension:

$$
1 \rightarrow \mathbb{C}^{*} \rightarrow \mathcal{H} / \mathcal{N} \rightarrow \mathcal{G}^{o} \rightarrow 1
$$

We now give an alternative description of the line bundle $\mathfrak{L}\left(\chi_{0}\right)$ on $X^{o}$ (cf. $\S$ C.6):

Recall the definition of the set $\mathcal{F}$ and the map $\beta: X^{o} \rightarrow \mathcal{F}$ from $\S($ C.7). For any $W \in \mathcal{F}$, define $S_{W}$ as the set of $\mathbb{C}$-linear isomorphisms $\theta: L_{o} \rightarrow W$ such that $\pi_{1} \theta-I: L_{o} \rightarrow L_{o}$ has finite rank, where $\pi_{1}: V((t)) \rightarrow L_{o}$ is the projection on the $L_{o}$ factor with respect to the decomposition (1).

Define a vector space $\mathcal{V}_{W}$ over $\mathbb{C}$ with basis parametrized by the set $S_{W}$, i.e., an element of $\mathcal{V}_{W}$ is of the form $\sum_{\theta \in S_{W}} z_{\theta} \theta$, where all but finitely many $z_{\theta}$ are zero. Let $\mathcal{V}_{W}^{\prime} \subset \mathcal{V}_{W}$ be the subspace spanned by $\left\{\theta-\operatorname{det}\left(\theta \theta^{\prime}-1\right) \theta^{\prime}\right\}_{\theta, \theta^{\prime} \in S_{W}}$ and let $\mathcal{L}_{W}=\mathcal{V}_{W} / \mathcal{V}_{W}^{\prime}$. (Note that $\theta \theta^{\prime}-1-I$ has finite rank as an endomorphism of $L_{o}$ and hence $\operatorname{det}\left(\theta \theta^{\prime}-1\right)$ is well defined.) Then $\mathcal{L}_{W}$ is a 1-dimensional vector space. Now define the line bundle $\mathcal{L} \stackrel{\eta}{\rightarrow} \mathcal{F}$, where $\eta^{-1}(W)=\mathcal{L}_{W}$ for any $W \in \mathcal{F}$. As proved in [PS, §7.7], the line bundle $\mathcal{L}$ is an algebraic line bundle on $\mathcal{F}$ (with respect to the ind-variety structure on $\mathcal{F}$ as in $\S$ C.7). It is easy to see that $\mathcal{L}_{\left.\right|_{\mathcal{F}_{1}}}$ is the restriction of the basic (negative ample) line bundle on $\operatorname{Gr}(n N, 2 n N)$ under the identification $\mathcal{F}_{1} \stackrel{\sim}{\rightarrow} G r(n N, 2 n N)^{1+\bar{t}_{1}}$ (cf. $\S$ C.7). Let $\mathcal{L}_{o}$ be the pull-back of the line bundle $\mathcal{L}$ to $X^{o}$ via the isomorphism $\beta: X^{o} \stackrel{\sim}{\rightarrow} \mathcal{F}$. In view of Proposition (C.13), it is easy to see that the dual line bundle $\mathcal{L}_{o}^{*}$ is isomorphic with the line bundle $\mathfrak{L}\left(\chi_{0}\right)$.

Now we define an action $\alpha$ of the group $\mathcal{H} / \mathcal{N}$ on $\mathcal{L}$ as follows: For $(g, E) \in \mathcal{H}$, define

$$
\alpha(g, E)[z, \theta]_{W}=\left[z, g \theta E^{-1}\right]_{g W},
$$

where for $z \in \mathbb{C}$ and $\theta \in S_{W},[z, \theta]_{W}$ denotes the equivalence class of $z \theta$. This action factors through an action of $\mathcal{H} / \mathcal{N}$ and moreover for any fixed $(g, E) \in \mathcal{H}, \alpha(g, E)$ is an algebraic automorphism of the line bundle $\mathcal{L}$ (and hence of $\mathcal{L}_{o}$ ) inducing the map $L_{g}$ on the base (cf. §C.6). Using this, the group $\mathcal{H} / \mathcal{N}$ can canonically be identified with the Mumford group $\operatorname{Aut}\left(\mathfrak{L}_{o}\right)$ defined in $\S($ C.6). In particular, the 
Finally we construct a splitting of $\mathcal{H} / \mathcal{N}$ over $\Gamma$ as follows:

Choose an element $g_{o} \in \mathcal{G}^{o}$ such that the associated rank- $N$ vector bundle $\tilde{\varphi}\left(g_{o}\right)$ on $C$ twisted by $\mathcal{O}((g-1) p), E:=\tilde{\varphi}\left(g_{o}\right)((g-1) p)$ (where $g$ is the genus of the curve $C$ ) has all its cohomology 0 . Then considering the local cohomology sequence (for the curve $C$ with support in $p$ ) with coefficients in the vector bundle $E$, we deduce that

$$
V((t))=L_{o} \oplus t^{1-g} g_{o}^{-1}\left(V \otimes_{\mathbb{C}} \mathbb{C}\left[C^{*}\right]\right)
$$

where $V \otimes_{\mathbb{C}} \mathbb{C}\left[C^{*}\right]$ is identified as a subspace of $V((t))$ by choosing a parameter $t$ around $p \in C$.

We first construct the splitting of $\mathcal{H} / \mathcal{N}$ over $\Gamma_{g_{o}}:=g_{o}^{-1} \Gamma g_{o}$. Define the group

homomorphism $\sigma_{g_{o}}: \Gamma_{g_{o}} \rightarrow \mathcal{H}$ by $\sigma_{g_{o}}(\gamma)=\left(\gamma, \gamma^{+^{\prime}}\right)$, where $\gamma=\left(\begin{array}{cc}\gamma^{+^{\prime}} & 0 \\ * & *\end{array}\right)$ with respect to the decomposition (3). (Observe that $\Gamma_{g_{o}}$ keeps the second factor stable and hence $\gamma^{+^{\prime}} \in G L\left(L_{o}\right)$.) The group homomorphism $\bar{\sigma}_{g_{o}}: \Gamma_{g_{o}} \rightarrow \mathcal{H} / \mathcal{N}$ (where $\bar{\sigma}_{g_{o}}$ is the map $\sigma_{g_{o}}$ followed by the canonical map $\left.\mathcal{H} \rightarrow \mathcal{H} / \mathcal{N}\right)$ splits the central extension (2) over $\Gamma_{g_{o}}$. Now take any preimage $\bar{g}_{o}$ of $g_{o}$ in $\mathcal{H} / \mathcal{N}$, and define the splitting $\bar{\sigma}: \Gamma \rightarrow \mathcal{H} / \mathcal{N}\left(\gamma \mapsto \bar{g}_{o} \bar{\sigma}_{g_{o}}\left(g_{o}^{-1} \gamma g_{o}\right) \bar{g}_{o}^{-1}\right)$.

Since $\mathcal{H} / \mathcal{N}$ acts on the line bundle $\mathcal{L}_{o}$, so is $\Gamma$ (via the homomorphism $\bar{\sigma}$ ). It can be easily seen that the action $\Gamma \times \mathcal{L}_{o} \rightarrow \mathcal{L}_{o}$ is a morphism of ind-varieties. Moreover, let $\bar{\sigma}^{\prime}: \Gamma \rightarrow \mathcal{H} / \mathcal{N}$ be another splitting of $\Gamma$ such that the induced action $\Gamma \times \mathcal{L}_{o} \rightarrow \mathcal{L}_{O}$ is again a morphism of ind-varieties. Then we claim that $\bar{\sigma}^{\prime}=\bar{\sigma}$ : There is a group homomorphism $\alpha: \Gamma \rightarrow \mathbb{C}^{*}$ such that (cf.(2)) $\bar{\sigma}^{\prime}=\alpha \bar{\sigma}$. Further $\alpha$ is a morphism of ind-varieties (since the action of $\Gamma$ on $\mathcal{L}_{o}$ in both the cases is regular). But then $\alpha$ is identically 1 (cf. Remark 6.8(c)). This proves the uniqueness of such a splitting.

Since the line bundle $\mathcal{L}_{o}$ is isomorphic with the homogeneous line bundle $\mathfrak{L}\left(-\chi_{0}\right)$, it is easy to see that the group $\Gamma$ acts morphically on the representation $L(\mathbb{C}, 1)$ and hence on any $L(\mathbb{C}, d)$ (for $d \geq 0$, where $L(\mathbb{C}, d)$ is the irreducible representation of the affine Lie algebra $s \tilde{l}_{N}$ with central charge $d$, cf. $\S$ A.2).

(2.7) Splitting of the central extension over $\Gamma$ (general case). We now come to the case of general $G$ as in $\S 1.1$. Take a finite dimensional representation $V$ of $G$ such that the group homomorphism $\varphi: G \rightarrow \operatorname{SL}(V)$ has finite kernel, and consider the induced map at the Lie algebra level $d \varphi: \mathfrak{g} \rightarrow \operatorname{sl}(V)$. We denote the Lie algebra $s l(V)$ by $\mathfrak{g}^{o}$. The Lie algebra homomorphism $d \varphi$ induces a Lie algebra homomorphism $\tilde{\varphi}: \tilde{\mathfrak{g}} \rightarrow \tilde{\mathfrak{g}}^{o}$ defined by (cf. $\S \mathrm{A} .1$ )

$$
X \otimes p \mapsto(d \varphi(X)) \otimes p, \quad \text { and } K \mapsto m_{V} K^{o}
$$

for $X \in \mathfrak{g}$ and $p \in \mathbb{C}\left[t^{ \pm 1}\right]$; where $K$ (resp. $K^{o}$ ) is the canonical central element of $\tilde{\mathfrak{g}}$ (resp. $\tilde{\mathfrak{g}}^{o}$ ), and $m_{V}$ is the Dynkin index of the representation $V$ (cf. $§ 5.1$ ).

To distinguish the objects corresponding to $\operatorname{SL}(V)$ from that of $G$, we denote the former by a superscript $o$. Let us consider the irreducible representation $L^{o}(\mathbb{C}, 1)$ for the Lie algebra $\tilde{\mathfrak{g}}^{o}$ with central charge 1 and restrict it to the Lie algebra $\tilde{\mathfrak{g}}$ via the homomorphism $\tilde{\varphi}$. It can be seen that the $\tilde{\mathfrak{g}}$-submodule of $L^{o}(\mathbb{C}, 1)$ generated by the highest weight vector $v_{o}$ is isomorphic with $L\left(\mathbb{C}, m_{V}\right)$.

The representation $\varphi$ also gives rise to a morphism of the corresponding affine 
that the basic homogeneous line bundle $\mathfrak{L}^{o}\left(\chi_{0}\right)$ on $X^{o}$ pulls-back to the line bundle $\mathfrak{L}\left(m_{V} \chi_{0}\right)$ on $X$. In particular, the group $\Gamma$ acts morphically on the line bundles $\mathfrak{L}\left(d m_{V} \chi_{0}\right)$ (for any $\left.d \in \mathbb{Z}\right)$ and hence $\Gamma$ also acts morphically on the representation space $L\left(\mathbb{C}, d m_{V}\right)$.

Finally we have the following proposition, asserting that $X=\mathcal{G} / \mathcal{P}$ supports an algebraic family.

(2.8) Proposition. (a) There is an algebraic $G$-bundle $\mathcal{U} \rightarrow C \times X$ such that for any $x \in X$ the $G$-bundle $\mathcal{U}_{x}:=\mathcal{U}_{\mid C \times x}$ is isomorphic with $\varphi(x)$ (where $\varphi$ is the map of $\S 1.4)$. Moreover, the bundle $\mathcal{U}_{\mid C^{*} \times X}$ comes equipped with a trivialization $\alpha: \epsilon \stackrel{\sim}{\rightarrow} \mathcal{U}_{\mid C^{*} \times X}$, where $\epsilon$ is the trivial $G$-bundle on $C^{*} \times X$.

(b) Let $\mathcal{E} \rightarrow C \times Y$ be an algebraic family of $G$-bundles (parametrized by an algebraic variety $Y$ ), such that $\mathcal{E}$ is trivial over $C^{*} \times Y$ and also over $\left(\operatorname{Spec} \hat{\mathcal{O}}_{p}\right) \times Y$. Then, if we choose a trivialization $\beta: \epsilon^{\prime} \stackrel{\sim}{\rightarrow} \mathcal{E}_{\mid C^{*} \times Y}$, we get a Schubert variety $X_{\mathfrak{w}}$ and a unique morphism $f: Y \rightarrow X_{\mathfrak{w}}$ together with a G-bundle morphism $\hat{f}: \mathcal{E} \rightarrow \mathcal{U}_{\mid C \times X_{\mathfrak{w}}}$ inducing the map $I \times f$ at the base such that $\hat{f} \circ \beta=\alpha \circ \theta$, where $\epsilon^{\prime}$ is the trivial bundle on $C^{*} \times Y$ and $\theta$ is the canonical G-bundle morphism $\epsilon^{\prime} \rightarrow \epsilon$ inducing the map $I \times f$ at the base.

Proof. Let $R$ be a $\mathbb{C}$-algebra and let $Y:=\operatorname{Spec} R$ be the corresponding scheme. Suppose $E \rightarrow C \times Y$ is a $G$-bundle with trivialisations $\alpha$ of $E$ over $C^{*} \times Y$ and $\beta$ of $E$ over $\left(\operatorname{Spec} \hat{\mathcal{O}}_{p}\right) \times Y$. Note that the fiber product $\left(C^{*} \times Y\right) \times_{C \times Y}\left(\operatorname{Spec} \hat{\mathcal{O}}_{p} \times Y\right)$ is canonically isomorphic with $\left(\operatorname{Spec} \hat{k}_{p}\right) \times Y$. Therefore the trivialisations $\alpha$ and $\beta$ give rise to an element $\alpha \beta^{-1} \in G\left(\hat{k}_{p} \otimes R\right)$. Conversely, given an element $g \in G\left(\hat{k}_{p} \otimes R\right)$, we can construct the family $E \rightarrow C \times$ Spec $R$ by taking the trivial bundles on $C^{*} \times Y$ and $\left(\operatorname{Spec} \hat{\mathcal{O}}_{p}\right) \times Y$ and glueing them via the element $g$. Moreover, if $g_{1}$ and $g_{2}$ are two elements of $G\left(\hat{k}_{p} \otimes R\right)$ such that $g_{2}=g_{1} h$ with $h \in G\left(\hat{\mathcal{O}}_{p} \otimes R\right)$, then $h$ induces a canonical isomorphism of the bundles corresponding to $g_{1}$ and $g_{2}$. All these assertions are easily verified.

Choose a local parameter $t$ around $p \in C$. Let $\mathrm{ev}_{\infty}: G\left(\mathbb{C}\left[t^{-1}\right]\right) \rightarrow G$ be the group homomorphism induced from the algebra homomorphism $\mathbb{C}\left[t^{-1}\right] \rightarrow \mathbb{C}$ taking $t^{-1} \mapsto 0$, and let $N^{-}:=\operatorname{ker}\left(\mathrm{ev}_{\infty}\right)$. Then the image $U^{-}$of $N^{-}$in $X$ under the map $i: N^{-} \rightarrow X$ taking $g \mapsto g \mathcal{P}$ is an open subset of $X$. To construct a family of $G$-bundles on $X$, we first construct a family on the open sets $w U^{-} \subset X$, for $w \in \operatorname{Mor}_{1}\left(\mathbb{C}^{*}, T\right)$ as follows (cf. proof of Lemma C.10 for the notation $\operatorname{Mor}_{1}\left(\mathbb{C}^{*}, T\right)$ ):

From the discussion in the first paragraph, it suffices to construct an element $\theta_{w} \in G\left(\hat{k}_{p} \otimes k\left[w U^{-}\right]\right)$such that for every $w x \in w U^{-}$, the element $\theta_{w}$ evaluated at $w x$ (i.e. the image of $\theta_{w}$ under the evaluation map $G\left(\hat{k}_{p} \otimes k\left[w U^{-}\right]\right) \rightarrow G\left(\hat{k}_{p}\right)$ at $w x)$ satisfies $\theta_{w}(w x)=w i^{-1}(x) \bmod \mathcal{P}$. But, by definition, $N^{-} \subset G\left(\mathbb{C}\left[t^{-1}\right]\right)$ and hence we get a tautological map $\theta:\left(\mathbb{P}^{1}(\mathbb{C}) \backslash 0\right) \times N^{-} \rightarrow G$. It is easy to see that $\theta$ is a morphism under the ind-variety structure on $N^{-}$. (Observe that $U^{-}$being an open subset of $X_{\text {lat }}$ has an ind-variety structure and hence $N^{-}$acquires an indvariety structure via the bijection $i$.) Think of $\mathbb{C}^{*}=\mathbb{P}^{1}(\mathbb{C}) \backslash\{0, \infty\}$ and define $\bar{\theta}_{w}$ : $\mathbb{P}^{1}(\mathbb{C}) \backslash\{0, \infty\} \times w U^{-} \rightarrow G$ by $\bar{\theta}_{w}(z, w i(g))=w(z) \theta(z, g)$, for $z \in \mathbb{P}^{1}(\mathbb{C}) \backslash\{0, \infty\}$ and $g \in N^{-}$. The morphism $\bar{\theta}_{w}$ of course gives rise to an element $\theta_{w} \in G\left(\hat{k}_{p} \otimes k\left[w U^{-}\right]\right)$, and hence a $G$-bundle on $C \times w U^{-}$. 
to give a bundle on $C \times X$, it suffices to show that the map

$$
\bar{\theta}_{v}^{-1} \bar{\theta}_{w}: \mathbb{P}^{1}(\mathbb{C}) \backslash\{0, \infty\} \times\left(w U^{-} \cap v U^{-}\right) \rightarrow G
$$

extends to a morphism (again denoted by) $\bar{\theta}_{v}^{-1} \bar{\theta}_{w}: \mathbb{P}^{1}(\mathbb{C}) \backslash\{\infty\} \times\left(w U^{-} \cap v U^{-}\right) \rightarrow$ $G$ : But for any fixed $x \in w U^{-} \cap v U^{-}$, the map $\bar{\theta}_{v}^{-1} \bar{\theta}_{w}: \mathbb{P}^{1}(\mathbb{C}) \backslash\{0, \infty\} \times x \rightarrow G$ in fact is an element of $\mathcal{P}=G\left(\widehat{\mathcal{O}}_{p}\right)$, i.e., $\bar{\theta}_{v}^{-1} \bar{\theta}_{w}$ does not have a pole at 0 , for any fixed $x \in w U^{-} \cap v U^{-}$. From this it is easy to see that $\bar{\theta}_{v}^{-1} \bar{\theta}_{w}$ extends to a morphism $\mathbb{P}^{1}(\mathbb{C}) \backslash\{\infty\} \times\left(w U^{-} \cap v U^{-}\right) \rightarrow G$. Clearly the maps $\bar{\theta}_{v}^{-1} \bar{\theta}_{w}$ satisfy the 'cocycle condition' and hence we get a $G$-bundle on the whole of $C \times X$.

To prove the (b) part, let us choose a trivialization $\tau$ of the bundle $\mathcal{E}$ restricted to $\left(\operatorname{Spec} \hat{\mathcal{O}}_{p}\right) \times Y$. As above, this (together with the trivialization $\beta$ ) gives rise to a map $f_{\tau}: Y \rightarrow \mathcal{G}$ and hence a map $f: Y \rightarrow X$. (It is easy to see that the map $f$ does not depend upon the choice of the trivialization $\tau$.) We claim that there exists a large enough $X_{v}$ such that $\operatorname{Im} f \subset X_{\mathfrak{w}}$ and moreover $f: Y \rightarrow X_{\mathfrak{w}}$ is a morphism:

For both of these assertions, we can assume that $Y$ is an affine variety $Y=$ Spec $R$, for some $\mathbb{C}$-algebra $R$. Then the map $f_{\tau}$ can be thought of as an element (again denoted by) $f_{\tau} \in G\left(\hat{k}_{p} \otimes R\right)$. Choose an imbedding $G \hookrightarrow S L_{N}$. Then we can write $f_{\tau}=\left(f_{\tau}^{i, j}\right)_{1 \leq i, j \leq N}$, with $f_{\tau}^{i, j} \in \hat{k}_{p} \otimes R$. In particular, there exists a large enough $l \geq 0$ such that (for any $1 \leq i, j \leq N) f_{\tau}^{i, j} \in t^{-l} \mathbb{C}[[t]] \otimes R$. From this (together with Lemma C.10) one can see that $\operatorname{Im} f$ is contained in a Schubert variety $X_{\mathfrak{w}}$. Now the assertion that $f: Y \rightarrow X_{\mathfrak{w}}$ is a morphism follows from the description of the map $f^{\tau}$ as an element of $G\left(\hat{k}_{p} \otimes R\right)$ together with the description of the variety structure $X_{\text {lat }}$ on $X$. The remaining assertions of (b) are easy to verify, thereby completing the proof of (b).

\section{Preliminaries on moduli space of $G$-bundles and the determinant bundle.}

Throughout this section, we allow $G$ to be a connected reductive group over an algebraically closed field $k$ of char. 0 .

We recall some basic concepts and results on semistable $G$-bundles on $C$. The references are $[\mathrm{NS}],\left[\mathrm{R}_{1}\right],\left[\mathrm{R}_{2}\right]$, and $[\mathrm{RR}]$. Recall the definition of $G$-bundles and reduction of structure group from $\S 1.2$.

(3.1) Definition. Let $E \rightarrow C$ be a $G$-bundle. Then $E$ is said to be semistable (resp. stable), if for any reduction of structure group $E_{P}$ to any parabolic subgroup $P \subset G$ and any non-trivial character $\chi: P \rightarrow \mathbb{G}_{m}$ which is dominant with respect to some Borel subgroup contained in $P$, the degree of the associated line bundle $E_{P}(\chi)$ is $\leq 0$ (resp. $<0$ ). (Note that, by definition, a dominant character is taken to be trivial on the connected component of the centre of $G$.)

(3.2) Remark. When $G=G L_{n}$, this definition coincides with the usual definition of semistability (resp. stability) due to Mumford (cf. [NS]) vz. a vector bundle $V \rightarrow C$ is semistable (resp. stable) if for every subbundle $W \varsubsetneqq V$, we have $\mu(W) \leq \mu(V)$ 
Let $V \rightarrow C$ be a semistable vector bundle. Then there exists a filtration by subbundles

$$
V_{0}=0 \varsubsetneqq V_{1} \varsubsetneqq V_{2} \varsubsetneqq \cdots \varsubsetneqq V
$$

such that $\mu\left(V_{i}\right)=\mu(V)$ and $V_{i} / V_{i-1}$ are stable. Though such a filtration in general is not unique, the associated graded

$$
\operatorname{gr} V:=\underset{i \geq 1}{\bigoplus} V_{i} / V_{i-1}
$$

is uniquely determined by $V$ (upto an isomorphism).

We will now describe the corresponding notion of $\operatorname{gr} E$ for a semistable $G$-bundle E.

(3.3) Definition. A reduction of structure group of a $G$-bundle $E \rightarrow C$ to a parabolic subgroup $P$ is called admissible if for any character of $P$, which is trivial on the connected component of the centre of $G$, the associated line bundle of the reduced $P$-bundle has degree 0 .

It is easy to see that if $E_{P}$ is an admissible reduction of structure group to a parabolic subgroup $P$, then $E$ is semistable if and only if the $P / U$-bundle $E_{P}(P / U)$ is semistable, where $U$ is the unipotent radical of $P$. Moreover, a semistable $G$ bundle $E$ admits an admissible reduction to some parabolic subgroup $P$ such that $E_{P}(P / U)$ is, in fact, a stable $P / U$-bundle. Let $M$ be a Levi component of $P$. Then $M \approx P / U$ (as algebraic groups) and thus we get a stable $M$-bundle $E_{P}(M)$. Extend the structure group of this $M$-bundle to $G$ to get a semistable $G$-bundle denoted by $\operatorname{gr}(E)$. Then $\operatorname{gr}(E)$ is uniquely determined by $E$ (up to an isomorphism).

Two semistable $G$-bundles $E_{1}$ and $E_{2}$ are said to be $S$-equivalent if $\operatorname{gr}\left(E_{1}\right) \approx$ $\operatorname{gr}\left(E_{2}\right)$. We call a semistable $G$-bundle $E$ quasistable if $E \approx \operatorname{gr}(E)$. (It can be seen that a semistable vector bundle is quasistable if and only if it is a direct sum of stable vector bundles with the same $\mu$.)

Two $G$-bundles $E_{1}$ and $E_{2}$ on $C$ are said to be of the same topological type if they are isomorphic as $G$-bundles in the topological category. The topological types of all the algebraic $G$-bundles are bijectively parametrized by $\pi_{1}(G)$ (cf. $\left[\mathrm{R}_{2}, \S 5\right]$ ).

(3.4) Theorem. The set $\mathfrak{M}$ of $S$-equivalence classes of all the semistable $G$ bundles of a fixed topological type admits the structure of a normal, irreducible, projective variety over $k$, making it into a coarse moduli.

In particular, for any algebraic family $\mathcal{E} \rightarrow C \times Y$ of semistable $G$-bundles of the same topological type (parametrized by a variety $Y$ ), the set map $\beta: Y \rightarrow \mathfrak{M}$, which takes $y \in Y$ to the $S$-equivalence class of $\mathcal{E}_{y}$ in $\mathfrak{M}$ is a morphism.

The details can be found in $[\mathrm{NS}],\left[\mathrm{R}_{1}\right],\left[\mathrm{R}_{2}\right], \ldots$

(3.5) Lemma. Let $H$ be a connected affine algebraic group and $C$ a smooth projective curve over $k$. Then any principal $H$-bundle on $C$ is locally trivial in the Zariski topology.

Proof. Let $E$ be a principal $H$-bundle on $C$ and $U$ the unipotent radical of $H$. Since the group $M=H / U$ is connected and reductive, the $M$-bundle $E(M)$, obtained from $E$ by extension of structure group to $M$, is locally trivial in the Zariski topology $\left[\mathrm{R}_{3}\right.$, Proposition 4.3].

Let $W$ be a non-empty affine open subset of $C$ such that the restriction of $E(M)$ 
that a trivialisation of $E(M)$ on $W$ gives a reduction of the structure group $H$ of $E_{\mid W}$ to the subgroup $U$. So, it suffices to show that any (principal) $U$-bundle on $W$ is trivial:

We may assume $U \neq e$. Then there exists a (finite) filtration of $U$ by closed normal subgroups such that the successive quotients are isomorphic to the additive group $G_{a}$. Now the assertion follows since any principal $G_{a}$-bundle on $W$ is trivial, $W$ being affine (see $\left[\mathrm{Se}_{1}, \S 5.1\right]$ ).

Let $P$ be a parabolic subgroup of $G$ and $P=M U$ a Levi decomposition, where $U$ is the unipotent radical of $P$ and $M$ a Levi component. The next proposition will be used in $\S 6$ in the case of an admissible reduction of a semistable bundle $E$.

(3.6) Proposition. Let $G$ be a connected semisimple algebraic group. Let $E$ be $a G$-bundle on $C$ and $E_{P}$ a reduction of the structure group of $E$ to $P$. Denote by $\operatorname{gr}\left(E_{P}\right)$ the $G$-bundle on $C$ obtained from the P-bundle $E_{P}$ by extension of the structure group via the composite homomorphism

$$
P \rightarrow P / U \approx M \hookrightarrow G .
$$

Then there exists a $G$-bundle $\mathcal{E}$ on $C \times \mathbb{A}^{1}$, where $\mathbb{A}^{1}$ is the affine line, such that we have

(a) $\mathcal{E}_{\mid C \times\left(\mathbb{A}^{1} \backslash 0\right)} \approx p_{C}^{*}(E), \mathcal{E}_{\mid C \times\{0\}} \approx g r\left(E_{P}\right)$ and

(b) $\mathcal{E}_{\mid C^{*} \times \mathbb{A}^{1}}$ is trivial and also the pull-back of $\mathcal{E}$ to $\left(\right.$ Spec $\left.\hat{\mathcal{O}}_{p}\right) \times \mathbb{A}^{1}$ is trivial, where $p_{C}$ is the projection on the $C$-factor.

Proof. By $\left[\mathrm{R}_{1}\right.$, Lemma 2.5.12], there exists a one-parameter group $\lambda: G_{m}(:=$ $\left.\mathbb{A}^{1} \backslash 0\right) \rightarrow M$, such that the regular map

$$
G_{m} \times P \rightarrow P \text {, given by }(t, p) \mapsto \lambda(t) p \lambda(t)^{-1} \text {, for } t \in G_{m}, p \in P \text {, }
$$

extends to a regular map $\phi: \mathbb{A}^{1} \times P \rightarrow P$ satisfying $\phi(0, m u)=m$, for $m \in M, u \in$ $U$. By Lemma (3.5), the $P$-bundle $E_{P}$ is locally trivial in the Zariski topology. Let $\left\{U_{i}\right\}$ be an affine open covering of $C$ in which the bundle $E_{P}$ is given by the transition functions $p_{i j}: U_{i} \cap U_{j} \rightarrow P$. Let $\mathcal{F}$ be the (Zariski locally trivial) $P$-bundle on $C \times \mathbb{A}^{1}$ defined by the covering $\left\{U_{i} \times \mathbb{A}^{1}\right\}$ and the transition functions

$$
h_{i j}:\left(U_{i} \cap U_{j}\right) \times \mathbb{A}^{1} \rightarrow P,
$$

where $h_{i j}(z, t)=\phi\left(t, p_{i j}(z)\right)$, for $t \in \mathbb{A}^{1}, z \in U_{i} \cap U_{j}$. Now let $\mathcal{E}$ be the $G$-bundle obtained from the $P$-bundle $\mathcal{F}$ by extension of the structure group to $G$. Then clearly $\mathcal{E}$ satisfies condition (a).

We next show that for any non-empty affine open subset $W$ of $C$, the restriction of $\mathcal{E}$ to $W \times \mathbb{A}^{1}$ is trivial (this will, in particular, imply that condition b is satisfied): Note that, by our construction, there exists a finite open covering $W_{i}$ of $W$ such that $\mathcal{E}_{\mid W_{i} \times \mathbb{A}^{1}}$ is trivial, for every $i$. Now by an analogue of a result of Quillen (cf. [Ra, Theorem 2]) $\mathcal{E}_{\mid W \times \mathbb{A}^{1}}$ is the pull-back of a $G$-bundle on $W$. But by Proposition (1.3), any $G$-bundle on $W$ is trivial.

(3.7) Determinant bundle and $\Theta$-bundle. We now briefly recall a few definitions and facts on the determinant bundles and $\Theta$-bundles associated to families of bundles 
Let $\mathcal{V} \rightarrow C \times Y$ be a vector bundle. Then there exists a complex of vector bundles $\mathcal{V}_{i}$ on $Y$ ( with $\mathcal{V}_{i}=0$, for all $i \geq 2$ ):

$$
\mathcal{V}_{0} \rightarrow \mathcal{V}_{1} \rightarrow 0 \rightarrow 0 \rightarrow \ldots
$$

such that for any base change $f: Z \rightarrow Y$, the $i^{\text {th }}$ direct image (under the projection $C \times Z \rightarrow Z$ ) of the pull-back $(\mathrm{id} \times f)^{*} \mathcal{V}$ on $Z$ is given by the $i^{\text {th }}$ cohomology of the pull-back of the above complex to $Z$. We define the determinant line bundle Det $\mathcal{V}$ on $Y$ to be the product $\wedge^{\text {top }}\left(\mathcal{V}_{1}\right) \otimes\left(\wedge^{\text {top }}\left(\mathcal{V}_{0}\right)^{*}\right)$. ( Notice that our Det $\mathcal{V}$ is dual to the determinant line bundle as defined, e.g., in [L, Chapter $6, \S 1]$.

The above base change property gives rise to the base change property for Det $\mathcal{V}$, i.e., if $f: Z \rightarrow Y$ is a morphism then $\operatorname{Det}\left((\mathrm{id} \times f)^{*} \mathcal{V}\right)=f^{*}(\operatorname{Det} \mathcal{V})$.

Let $\mathfrak{L}$ be a line bundle on $Y$, and let $p_{2}: C \times Y \rightarrow Y$ be the projection on the second factor. Then for the family $\mathcal{V} \otimes p_{2}^{*} \mathfrak{L} \rightarrow C \times Y$, we have $\operatorname{Det}\left(\mathcal{V} \otimes p_{2}^{*} \mathfrak{L}\right)=$ $($ Det $\mathcal{V}) \otimes \mathfrak{L}^{-\mathcal{X}(\mathcal{V})}$, where $\mathcal{X}(\mathcal{V}):=h^{0}\left(\mathcal{V}_{t}\right)-h^{1}\left(\mathcal{V}_{t}\right)$ is the Euler characteristic and $\mathcal{V}_{t}:=\left.\mathcal{V}\right|_{C \times t}$. (Observe that $h^{0}\left(\mathcal{V}_{t}\right)-h^{1}\left(\mathcal{V}_{t}\right)$ remains constant on any connected component of $Y$.)

We now define the $\Theta$-bundle $\Theta(\mathcal{V})$ of a family of rank $r$ and degree 0 bundles $\mathcal{V} \rightarrow$ $C \times Y$ to be the modified determinant bundle given by $(\operatorname{Det} \mathcal{V}) \otimes\left(\operatorname{det}\left(\mathcal{V}_{p}\right)\right)^{\mathcal{X}(\mathcal{V}) / r}$, where $\mathcal{V}_{p}$ is the bundle $\left.\mathcal{V}\right|_{p \times Y}$ on $Y$, and det $\mathcal{V}_{p}$ is its usual determinant line bundle. It follows then that $\Theta(\mathcal{V})=\Theta\left(\mathcal{V} \otimes p_{2}^{*} \mathfrak{L}\right)$, for any line bundle $\mathfrak{L}$ on $Y$. Moreover $\Theta(\mathcal{V})$ also has the functorial property $\Theta\left((\mathrm{id} \times f)^{*} \mathcal{V}\right)=f^{*}(\Theta(\mathcal{V}))$.

If $\mathcal{E} \rightarrow C \times Y$ is a family of $G$-bundles (where $G$ is semisimple and connected) and $V$ is a $G$-module, then Det $(\mathcal{E}(V))$ and $\Theta(\mathcal{E}(V))$ are defined to be the corresponding line bundles of the associated family of vector bundles, via the representation $V$ of G.

For the family $\mathcal{U} \rightarrow C \times X$ (cf. Proposition 2.8), the line bundles $\Theta(\mathcal{U}(V))$ and $\operatorname{Det}(\mathcal{U}(V))$ coincide, since $\mathcal{U}_{\left.\right|_{p \times \mathcal{G} / \mathcal{P}}}$ is trivial.

It is known ([DN], $[\mathrm{NRa}])$ that there exists a line bundle $\Theta$ on the moduli space $\mathfrak{M}_{o}$ of rank $r$ and degree 0 (semistable) bundles, such that for any family $\mathcal{V}$ of rank $r$ and degree 0 semistable bundles parametrized by $Y$ we have $f^{*}(\Theta) \simeq \Theta(\mathcal{V})$, where $f: Y \rightarrow \mathfrak{M}_{o}$ is the morphism given by the coarse moduli property of $\mathfrak{M}_{o}$ (cf. Theorem 3.4).

Let $V$ be a representation of $G$ of dimension $r$ ( $G$ semisimple and connected). Then for any semistable $G$-bundle on $C$, the associated vector bundle (via the representation $V$ ) is semistable (cf. [RR, Theorem 3.18]). Thus, given a family of semistable $G$-bundles on $C$ parametrized by $Y$, we have a canonical morphism (induced from the representation $V$ ) $Y \rightarrow \mathfrak{M}_{o}$ (where $\mathfrak{M}_{o}$ as above is the moduli space of semistable bundles of rank $r$ and degree 0 ). Let $\mathfrak{M}$ be the moduli space of semistable $G$-bundles. By the coarse moduli property of $\mathfrak{M}$, we see that we have a canonical morphism $\phi_{V}: \mathfrak{M} \rightarrow \mathfrak{M}_{o}$. We define the theta bundle $\Theta(V)$ on $\mathfrak{M}$ associated to $V$ to be the pull-back of the line bundle $\Theta$ on $\mathfrak{M}_{o}$ via the morphism $\phi_{V}$. It can be easily seen that for any family $\mathcal{V} \rightarrow C \times Y$ of semistable $G$-bundles, $f^{*}(\Theta(V)) \simeq \Theta(\mathcal{V}(V))$, where $f: Y \rightarrow \mathfrak{M}$ is the morphism (induced from the family $\mathcal{V})$ given by the coarse moduli property of $\mathfrak{M}$. 
We prove the following technical result, which will crucially be used in the note. Even though we believe that it should be known, we did not find a precise reference.

(4.1) Proposition. Let $f: X \rightarrow Y$ be a surjective morphism between irreducible algebraic varieties $X$ and $Y$ over an algebraically closed field $k$ of char 0 . Assume that $Y$ is normal and let $\mathcal{E} \rightarrow Y$ be an algebraic vector bundle on $Y$.

Then any set theoretic section $\sigma$ of the vector bundle $\mathcal{E}$ is regular if and only if the induced section $f^{*}(\sigma)$ of the induced bundle $f^{*}(\mathcal{E})$ is regular.

Proof. The 'only if' part is of course trivially true. So we come to the 'if' part.

Since the question is local (in $Y$ ), we can assume that $Y$ is affine and moreover the vector bundle $\mathcal{E}$ is trivial, i.e., it suffices to show that any (set theoretic) map $\sigma: Y \rightarrow k$ is regular, provided $\bar{\sigma}:=\sigma \circ f: X \rightarrow k$ is regular (under the assumption that $Y=\operatorname{Spec} R$ is irreducible normal and affine):

Since the map $f$ is surjective (in particular dominant), the ring $R$ is canonically embedded in $\Gamma(X):=H^{0}\left(X, \mathcal{O}_{X}\right)$. Let $R[\bar{\sigma}]$ denote the subring of $\Gamma(X)$ generated by $R$ and $\bar{\sigma} \in \Gamma(X)$. Then $R[\bar{\sigma}]$ is a (finitely generated) domain (as $X$ is irreducible by assumption), and we get a dominant morphism $\hat{f}: Z \rightarrow$ Spec $R$, where $Z:=$ Spec $(R[\bar{\sigma}])$. Consider the commutative diagram:

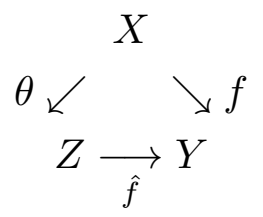

where $\theta$ is the dominant morphism induced from the inclusion $R[\bar{\sigma}] \hookrightarrow \Gamma(X)$. In particular, Im $\theta$ contains a non-empty Zariski open subset $U$ of $Z$. Let $x_{1}, x_{2} \in X$ be closed points such that $f\left(x_{1}\right)=f\left(x_{2}\right)$. Then $r\left(x_{1}\right)=r\left(x_{2}\right)$, for all $r \in R$ and also $\bar{\sigma}\left(x_{1}\right)=\bar{\sigma}\left(x_{2}\right)$. This forces $\theta\left(x_{1}\right)=\theta\left(x_{2}\right)$, in particular, $\hat{f}_{\mid U}$ is injective on the closed points of $U$.

Since $\hat{f}$ is dominant, by cutting down $U$ if necessary, we can assume that $\hat{f}_{\mid U}$ : $U \rightarrow V$ is a bijection, for some open subset $V \subset Y$. Now since $Y$ is (by assumption) normal and $Z$ is irreducible, by Zariski's main theorem (cf. [Mum, Page 288, I. Original form]), $\hat{f}_{\mid U}: U \rightarrow V$ is an isomorphism, and hence $\sigma$ is regular on $V$.

Assume, if possible, that $\sigma_{\left.\right|_{V}}$ does not extend to a regular function on the whole of $Y$. Then, by [B, Lemma 18.3, Chapter AG], there exists a point $y_{0} \in Y$ and a regular function $h$ on a Zariski neighborhood $W$ of $y_{0}$ such that $h\left(y_{0}\right)=0$ and $h \sigma=1$ on $W \cap V$. But then $\bar{h} \bar{\sigma}=1$ on $f^{-1}(W \cap V)$ (where $\left.\bar{h}:=h \circ f\right)$ and hence, $\bar{\sigma}$ being regular on the whole of $X, \bar{h} \bar{\sigma}=1$ on $f^{-1}(W)$. Taking $\overline{y_{0}} \in f^{-1}\left(y_{0}\right)$ ( $f$ is, by assumption, surjective), we get $\bar{h}\left(\overline{y_{0}}\right) \bar{\sigma}\left(\overline{y_{0}}\right)=0$. This contradiction shows that $\sigma_{\left.\right|_{V}}$ does extend to some regular function (say $\sigma^{\prime}$ ) on the whole of $Y$. Hence $\bar{\sigma}=\bar{\sigma}^{\prime}$, in particular, by the surjectivity of $f, \sigma=\sigma^{\prime}$. This proves the proposition.

\section{Identification of the determinant bundle.}

(5.1) Recall from $\S 2.8$ that $\mathcal{G} / \mathcal{P}$ is a parameter space for an algebraic family $\mathcal{U}$ of $G$-bundles on $C$. Let us fix a (finite dimensional) representation $V$ of $G$. In 
recall the definition of the fundamental homogeneous line bundle $\mathfrak{L}\left(\chi_{o}\right)$ on $\mathcal{G} / \mathcal{P}$ from $\S($ C.6). Our aim in this section is to determine the line bundle $\operatorname{Det}(\mathcal{U}(V))$ in terms of $\mathfrak{L}\left(\chi_{o}\right)$. We begin with the following preparation.

Let $\theta$ be the highest root of $\mathfrak{g}$. Define the following Lie subalgebra $s l_{2}(\theta)$ of the Lie algebra $\mathfrak{g}$ of $G$ :

$$
s l_{2}(\theta):=\mathfrak{g}_{-\theta} \oplus \mathbb{C} \theta^{\vee} \oplus \mathfrak{g}_{\theta},
$$

where $\mathfrak{g}_{\theta}$ is the $\theta$-th root space, and $\theta^{\vee}$ is the corresponding coroot. Clearly $s l_{2}(\theta) \approx$ $s l_{2}$ as Lie algebras. Decompose

$$
V=\oplus_{i} V_{i},
$$

as a direct sum of irreducible $s l_{2}(\theta)$ - modules $V_{i}$ of $\operatorname{dim} m_{i}$. Now we define

$$
m_{V}=\sum_{i}\left(\begin{array}{c}
m_{i}+1 \\
3
\end{array}\right), \text { where we set }\left(\begin{array}{l}
2 \\
3
\end{array}\right)=0 .
$$

Let $\mathfrak{g}_{1}$ and $\mathfrak{g}_{2}$ be two (finite dimensional) complex simple Lie algebras and $\varphi$ : $\mathfrak{g}_{1} \rightarrow \mathfrak{g}_{2}$ be a Lie algebra homomorphism. There exists a unique number $m_{\varphi} \in \mathbb{C}$, called the Dynkin index (cf. [D, §2]) of the homomorphism $\varphi$, satisfying

$$
\langle\varphi(x), \varphi(y)\rangle=m_{\varphi}\langle x, y\rangle, \text { for all } x, y \in \mathfrak{g}_{1},
$$

where $\langle$,$\rangle is the Killing form on \mathfrak{g}_{1}$ (and $\mathfrak{g}_{2}$ ) normalized so that $\langle\theta, \theta\rangle=2$ for the highest root $\theta$.

It is easy to see from the next Lemma (5.2) that for a finite dimensional representation $V$ of $\mathfrak{g}_{1}$ given by a Lie algebra homomorphism $\varphi: \mathfrak{g}_{1} \rightarrow \operatorname{sl}(V)$, we have $m_{\varphi}=m_{V}$, where $s l(V)$ is the Lie algebra of trace 0 endomorphisms of $V$.

We give an expression for $m_{V}$ in the following lemma. Write the formal character

$$
\operatorname{ch} V=\sum n_{\lambda} e^{\lambda} .
$$

\section{(5.2) Lemma.}

$$
m_{V}=\frac{1}{2} \sum_{\lambda} n_{\lambda}<\lambda, \theta^{\vee}>^{2} .
$$

In particular, for the adjoint representation ad of $\mathfrak{g}$ we have

$$
m_{a d}=2\left(1+<\rho, \theta^{\vee}>\right),
$$

where $\rho$ as usual is the half sum of the positive roots of $\mathfrak{g}$.

Similarly, for the standard $n$-dim. representation $V^{n}$ of $s l_{n}, m_{V^{n}}=1$.

Proof. It suffices to show that, for the irreducible representation $W(m)$ (of dim $m+1)$ of $s l_{2}$

$$
\frac{1}{2} \sum^{m}<m \rho_{1}-n \alpha, H>^{2}=\left(\begin{array}{c}
m+2 \\
3
\end{array}\right),
$$


where $\alpha$ is the unique positive root of $s l_{2}, H$ the corresponding coroot and $\rho_{1}:=\frac{1}{2} \alpha$. Now the left side of (3) is equal to

$$
\begin{aligned}
2 \sum_{n=0}^{m}\left(\frac{m}{2}-n\right)^{2} & =4 \sum_{k=1}^{k_{o}} k^{2}=\frac{m(m+1)(m+2)}{6}, \text { if } m=2 k_{o} \text { is even, and } \\
2 \sum_{n=0}^{m}\left(\frac{m}{2}-n\right)^{2} & =2 \sum_{n=0}^{m}\left(k_{o}-\frac{1}{2}-n\right)^{2}, \text { if } m=2 k_{o}-1 \text { is odd } \\
& =4 \sum_{k=1}^{k_{o}}\left(k-\frac{1}{2}\right)^{2}=\left(4 \sum_{k=1}^{k_{o}} k^{2}\right)+k_{o}-4 \sum_{k=1}^{k_{o}} k \\
& =\frac{m(m+1)(m+2)}{6} .
\end{aligned}
$$

So in either case the left side of $(3)=\frac{m(m+1)(m+2)}{6}=\left(\begin{array}{c}m+2 \\ 3\end{array}\right)$. This proves the first part of the lemma.

For the assertion regarding the adjoint representation, we have

$$
\operatorname{ch}(a d)=\operatorname{dimh} \cdot e^{0}+\sum_{\beta \in \Delta_{+}}\left(e^{\beta}+e^{-\beta}\right) .
$$

So $\quad m_{a d}=\sum_{\beta \epsilon \Delta_{+}}<\beta, \theta^{\vee}>^{2}$

$$
\begin{aligned}
& =4+\sum_{\beta \in \Delta_{+} \backslash \theta}<\beta, \theta^{\vee}>\text {, since }<\beta, \theta^{\vee}>=0 \text { or } 1, \text { for any } \beta \in \Delta_{+} \backslash \theta \\
& =4+<2 \rho-\theta, \theta^{\vee}> \\
& =2\left(1+<\rho, \theta^{\vee}>\right) .
\end{aligned}
$$

The assertion about $m_{V^{n}}$ is easy to verify.

(5.3) Remark. The number $\left(1+<\rho, \theta^{\vee}>\right)$ is called the dual Coxeter number of $\mathfrak{g}$. Its value is given as below.

$\begin{array}{cc}\text { Type of } \mathfrak{g} & \text { dual Coxeter number } \\ A_{\ell} & \ell+1 \\ B_{\ell} & 2 \ell-1 \\ C_{\ell} & \ell+1 \\ D_{\ell} & 2 \ell-2 \\ E_{6} & 12 \\ E_{7} & 18 \\ E_{8} & 30 \\ G_{2} & 4 \\ F_{4} & 9\end{array}$


(5.4) Theorem. With the notation as in $\S 5.1$

$$
\operatorname{Det}(\mathcal{U}(V)) \simeq \mathfrak{L}\left(m_{V} \chi_{o}\right)
$$

for any finite dimensional representation $V$ of $G$, where the number $m_{V}$ is defined by (3) of $\S 5.1$.

Proof. By Proposition (C.13), there exists an integer $m$ such that

$$
\operatorname{Det}(\mathcal{U}(V)) \simeq \mathfrak{L}\left(m \chi_{o}\right) \in \operatorname{Pic}(\mathcal{G} / \mathcal{P})
$$

We want to prove that $m=m_{V}:$ Set $\mathcal{U}_{o}:=\mathcal{U}(V)_{\mid C \times X_{o}}$ as the family restricted to the Schubert variety $X_{o}:=X_{\mathfrak{s}_{o}}$ (cf. proof of Proposition C.13). Denote by $\alpha$ (resp. $\beta)$ the canonical generator of $H^{2}\left(X_{o}, \mathbb{Z}\right)\left(\right.$ resp. $\left.H^{2}(C, \mathbb{Z})\right)$. Then it suffices to show that Det $\mathcal{U}_{o} \simeq \mathfrak{L}\left(m_{V} \chi_{o}\right)_{\left.\right|_{X_{o}}}$, which is equivalent to showing that the first Chern class

$$
c_{1}\left(\operatorname{Det} \mathcal{U}_{o}\right)=m_{V} \alpha:
$$

From the definition of the determinant bundle we have

$$
c_{1}\left(\operatorname{Det} \mathcal{U}_{o}\right)=-c_{1}\left(\pi_{2 *} \mathcal{U}_{o}\right)
$$

where $\pi_{2}$ is the projection $C \times X_{o} \rightarrow X_{o}$, and the notation $\pi_{2 *}$ is as in [F, Chapter $9]$.

Since $G$ is semisimple, the associated vector bundle $\mathcal{U}(V)$ has

$$
c_{1}\left(\mathcal{U}_{o}\right)=0
$$

Let $\tilde{\alpha}(\operatorname{resp} . \tilde{\beta})$ be the pull back of $\alpha(\operatorname{resp} . \beta)$ under $\pi_{2}\left(\operatorname{resp} . \pi_{1}\right)$. Now write

$$
c_{2}\left(\mathcal{U}_{o}\right)=l \tilde{\alpha} \tilde{\beta}, \text { for some (unique) } l \in \mathbb{Z} \text {. }
$$

Let $T_{\pi_{2}}$ be the relative tangent bundle along the fibers of $\pi_{2}$. Let us denote by $c_{1}$ (resp. $c_{2}$ ) the first (resp. second) Chern class of $\mathcal{U}_{o}$. By the Grothendieck's Riemann-Roch theorem [F, §9.1] applied to the (proper) map $\pi_{2}$, we get

$$
\begin{aligned}
\operatorname{ch}\left(\pi_{2 *} \mathcal{U}_{o}\right) & =\pi_{2 *}\left(\operatorname{ch}\left(\mathcal{U}_{o}\right) \cdot \operatorname{td}\left(T_{\pi_{2}}\right)\right) \\
& =\pi_{2 *}\left[\left(\operatorname{rk} \mathcal{U}_{o}+c_{1}+\frac{1}{2}\left(c_{1}^{2}-2 c_{2}\right)\right)\left(1+\frac{1}{2} c_{1}\left(T_{\pi_{2}}\right)\right)\right] \\
& =\pi_{2 *}\left[\left(\operatorname{rk} \mathcal{U}_{o}-c_{2}\right)\left(1+\frac{1}{2} c_{1}\left(T_{\pi_{2}}\right)\right)\right], \text { by }(3),
\end{aligned}
$$

where ch denotes the Chern character and td denotes the Todd class. Hence

$$
\begin{aligned}
c_{1}\left(\pi_{2 *} \mathcal{U}_{o}\right) & =\pi_{2 *}\left(-c_{2}\left(\mathcal{U}_{o}\right)\right) \\
& =\pi_{2 *}(-l \tilde{\alpha} \tilde{\beta}), \text { by }(4)
\end{aligned}
$$


So to prove the theorem, by (1),(2) and (5), we need to show that $l=m_{V}$, where $l$ is given by (4):

It is easy to see (from its definition) that topologically the bundle $\mathcal{U}_{o}$ is pull-back of the bundle $\mathcal{U}_{o}^{\prime}\left(\right.$ Where $\mathcal{U}_{o}^{\prime}$ is the same as $\mathcal{U}_{o}$ for $C=\mathbb{P}^{1}$ ) on $\mathbb{P}^{1} \times X_{o}$ via the map

$$
C \times X_{o} \stackrel{\delta \times \mathrm{I}}{\rightarrow} \mathbb{P}^{1} \times X_{o}
$$

where $\delta: C \rightarrow \mathbb{P}^{1}$ pinches all the points outside a small open disc around $p$ to a point. Of course the map $\delta$ is of degree 1, so the cohomology generator $\alpha$ pulls back to the generator $\beta$. Hence it suffices to compute the second Chern class of the bundle $\mathcal{U}_{o}^{\prime}$ on $\mathbb{P}^{1} \times X_{o}$ :

Choose $X_{\theta} \in \mathfrak{g}_{\theta}$ (where $\theta$ is the highest root of $\mathfrak{g}$ ) such that $\left\langle X_{\theta},-\omega X_{\theta}\right\rangle=1$, where $\omega$ is the Cartan involution of $\mathfrak{g}$ and $<,>$ is the Killing form on $\mathfrak{g}$, normalized so that $\langle\theta, \theta\rangle=2$. Set $Y_{\theta}:=-\omega\left(X_{\theta}\right) \in \mathfrak{g}_{-\theta}$. Define a Lie algebra homomorphism $s l_{2} \rightarrow \mathfrak{g} \otimes_{\mathbb{C}} \mathbb{C}\left[t^{ \pm 1}\right]$, by

$$
\begin{aligned}
& X \quad \mapsto Y_{\theta} \otimes t \\
& Y \mapsto X_{\theta} \otimes t^{-1} \\
& H \quad \mapsto \quad-\theta^{\vee} \otimes 1,
\end{aligned}
$$

where $\{X, Y, H\}$ is the standard basis of $s l_{2}$. The corresponding group homomorphism (choosing a local parameter $t$ around $p$ ) $\eta: S L_{2}(\mathbb{C}) \rightarrow \mathcal{G}$ induces a biregular isomorphism $\bar{\eta}: \mathbb{P}^{1} \approx S L_{2}(\mathbb{C}) / B_{1} \sim \vec{\rightarrow} X_{o}$, where $B_{1}$ is the standard Borel subgroup of $S L_{2}(\mathbb{C})$ consisting of upper triangular matrices. In what follows we will identify $X_{o}$ with $\mathbb{P}^{1}$ under $\bar{\eta}$. The representation $V$ of $G$ on restriction, under the decomposition $(2)$ of $\S 5.1$, gives rise to a continuous group homomorphism

$$
\psi: S U_{2}(\theta) \rightarrow \prod_{i}\left(\mathrm{Aut} V_{i}\right)
$$

where $S U_{2}(\theta)$ is the standard compact form (induced from the involution $\omega$ ) of the group $S L_{2}(\theta)$ (with Lie algebra $s l_{2}(\theta)$ ).

There is a principal $S U_{2}$-bundle $\mathcal{W}$ on $S^{4}$ (in the topological category) got by the clutching construction from the identity map $S^{3} \approx S U_{2} \rightarrow S U_{2}$. In particular, we obtain the vector bundle $\mathcal{W}(\psi) \rightarrow S^{4}$ associated to the principal bundle $\mathcal{W}$ via the representation $\psi$, which breaks up as a direct sum of subbundles $\mathcal{W}_{i}(\psi)$ (got from the representations $V_{i}$ ).

We further choose a degree 1 continuous map $\nu: \mathbb{P}^{1} \times \mathbb{P}^{1} \rightarrow S^{4}$. We claim that the vector bundle $\mathcal{U}_{o}^{\prime}$ on $\mathbb{P}^{1} \times \mathbb{P}^{1}$ is isomorphic (in the topological category) with the pull back $\nu^{*}(\mathcal{W}(\psi))$ :

Define a map $\Phi: S^{1} \times\left(S U_{2} / D\right) \rightarrow S U_{2}$ by

$$
\left(t,\left(\begin{array}{ll}
a & b \\
c & d
\end{array}\right) \bmod D\right) \mapsto\left(\begin{array}{cc}
d c t^{-1} \\
b t a
\end{array}\right)\left(\begin{array}{ll}
d & c \\
b & a
\end{array}\right)^{-1}
$$

for $\left(\begin{array}{l}a b \\ c d\end{array}\right) \in S U_{2}$ and $t \in S^{1}$; where $D$ is the diagonal subgroup of $S U_{2}$. It is easy to see that the principal $S U_{2}$-bundle $\nu^{*}(\mathcal{W})$ on $\mathbb{P}^{1} \times \mathbb{P}^{1}$ is isomorphic with the 
covering $\mathbb{P}^{1} \times \mathbb{P}^{1}=S^{2} \times S^{2}=H^{+} \times S^{2} \cup H^{-} \times S^{2}$, where $H^{+}$and $H^{-}$are resp. the upper and lower closed hemispheres). By composing $\Phi$ with the isomorphism $S U_{2} \rightarrow S U_{2}(\theta)$ (induced from the Lie algebra homomorphism $s l_{2} \rightarrow s l_{2}(\theta)$ taking $X \mapsto X_{\theta}, Y \mapsto Y_{\theta}$, and $H \mapsto \theta^{\vee}$ ), and using the isomorphism $\bar{\eta}$ together with the definition of the vector bundle $\mathcal{U}_{o}$ we get the assertion that $\mathcal{U}_{o}^{\prime} \approx \nu^{*}(\mathcal{W}(\psi))$. So

$$
\begin{aligned}
c_{2}\left(\mathcal{U}_{o}^{\prime}\right) & =\nu^{*}\left(c_{2}(\mathcal{W}(\psi))\right)=\nu^{*} \sum_{i} c_{2}\left(\mathcal{W}_{i}(\psi)\right) \\
& \left.=\sum_{i}\left(\begin{array}{c}
m_{i}+1 \\
3
\end{array}\right) \tilde{\alpha} \tilde{\beta} \text {, by the following lemma (since } \nu \text { is a map of degree } 1\right) .
\end{aligned}
$$

Hence $l=\sum_{i}\left(\begin{array}{c}m_{i}+1 \\ 3\end{array}\right)=m_{V}$, proving the theorem modulo the next lemma.

(5.5) Lemma. Let $W(m)$ be the $(m+1)$-dimensional irreducible representation of $S U_{2}$ and let $\mathcal{W}(m)$ be the vector bundle on $S^{4}$ associated to the principal $S U_{2}$ bundle $\mathcal{W}$ on $S^{4}$ (defined in the proof of Theorem 5.4) by the representation $W(m)$ of $S U_{2}$. Then

$$
c_{2}(\mathcal{W}(m))=\left(\begin{array}{c}
m+2 \\
3
\end{array}\right) \Omega,
$$

where $\Omega$ is the fundamental cohomology generator of $H^{4}\left(S^{4}, \mathbb{Z}\right)$.

Proof. By the Clebsch -Gordan theorem (cf.[Hu, Page 126]), we have the following decomposition as $S U_{2}$-modules:

$$
W(m) \otimes W(1)=W(m+1) \oplus W(m-1), \quad \text { for any } m \geq 1 .
$$

In particular, the Chern character

$$
\operatorname{ch} \mathcal{W}(m) \cdot \operatorname{ch} \mathcal{W}(1)=\operatorname{ch} \mathcal{W}(m+1)+\operatorname{ch} \mathcal{W}(m-1) .
$$

Assume, by induction, that (1) is true for all $l \leq m$. (The validity of (1) for $l=1$ is trivial to see.) Then by (2) we get

$$
\begin{aligned}
\operatorname{ch} \mathcal{W}(m+1)= & \operatorname{ch} \mathcal{W}(m) \cdot \operatorname{ch} \mathcal{W}(1)-\operatorname{ch} \mathcal{W}(m-1) \\
= & \left((m+1) \cdot 1-c_{2} \mathcal{W}(m)\right)\left(2.1-c_{2} \mathcal{W}(1)\right) \\
& -\left(m .1-c_{2} \mathcal{W}(m-1)\right), \text { since } c_{1} \mathcal{W}(l)=0 \text { as it is a } S U_{2} \text {-bundle. }
\end{aligned}
$$

Hence by induction

$$
\operatorname{ch} \mathcal{W}(m+1)=\left((m+1) .1-\left(\begin{array}{c}
m+2 \\
3
\end{array}\right) \Omega\right)(2.1-\Omega)-\left(m .1-\left(\begin{array}{c}
m+1 \\
3
\end{array}\right) \Omega\right) .
$$

Writing $\operatorname{ch} \mathcal{W}(m+1)=(m+2) .1-c_{2} \mathcal{W}(m+1)$, and equating the coefficients from (3), we get

$$
\begin{aligned}
c_{2} \mathcal{W}(m+1) & =\left(2\left(\begin{array}{c}
m+2 \\
3
\end{array}\right)+m+1-\left(\begin{array}{c}
m+1 \\
3
\end{array}\right)\right) \Omega \\
& =\left(\begin{array}{c}
m+3 \\
3
\end{array}\right) \Omega .
\end{aligned}
$$

This completes the induction and hence proves the lemma.

Recall that for any connected complex simple group $G$, the third homotopy group 
(5.6) Corollary. For any representation $\rho$ of $G$ in a finite dimensional vector space $V$, the induced map $\pi_{3}(G) \rightarrow \pi_{3}(S L(V))$ is multiplication by the number $m_{V}$.

Proof. The representation $\rho: G \rightarrow S L(V)$ gives rise to a morphism $\tilde{\rho}: \mathcal{G} / \mathcal{P} \rightarrow$ $\mathcal{G}^{o} / \mathcal{P}^{o}$, where $\mathcal{G}^{o}:=S L(V)\left(\hat{k}_{p}\right)$ and $\mathcal{P}^{o}:=S L(V)\left(\hat{\mathcal{O}}_{p}\right)$. Moreover, the family $\mathcal{U}^{o}(V)$ parametrized by $\mathcal{G}^{\circ} / \mathcal{P}^{o}$ (got from the standard representation $\epsilon$ of $S L(V)$ in $V$ ) pulls-back to the family $\mathcal{U}(V)$ (parametrized by $\mathcal{G} / \mathcal{P}$ ). In particular, from the functoriality of the determinant bundle (cf.§3.7), Theorem(5.4), and Lemma (5.2), we see that the induced map $\tilde{\rho}^{*}: H^{2}\left(\mathcal{G}^{o} / \mathcal{P}^{o}, \mathbb{Z}\right) \rightarrow H^{2}(\mathcal{G} / \mathcal{P}, \mathbb{Z})$ is multiplication by the number $m_{V}$ (under the canonical identifications $H^{2}\left(\mathcal{G}^{\circ} / \mathcal{P}^{o}, \mathbb{Z}\right) \simeq \mathbb{Z} \simeq H^{2}(\mathcal{G} / \mathcal{P}, \mathbb{Z})$ ). But the flag variety $\mathcal{G} / \mathcal{P}$ is homotopic to the based loop group $\Omega_{e}(K)$ (where $K$ is a compact form of $G$ ), and similarly $\mathcal{G}^{o} / \mathcal{P}^{o}$ is homotopic to $\Omega_{e}(S U(V))$. In particular, by the Hurewicz's theorem and the long exact homotopy sequence corresponding to the fibration $\Omega_{e}(K) \rightarrow P(K) \rightarrow K$ (where $P(K)$ is the path space of $K$ consisting of the paths starting at the base point $e$ ), the corollary follows.

\section{Statement of the main theorem and its proof.}

(6.1) Definitions. Recall the definition of the homogeneous line bundle $\mathfrak{L}\left(m \chi_{o}\right)$ on $X:=\mathcal{G} / \mathcal{P}($ for any $m \in \mathbb{Z})$ from $\S\left(\right.$ C.6). Define, for any $p \in \mathbb{Z}$, (cf. $\left.\left[\mathrm{Ku}_{1}, \S 3.8\right]\right)$

$$
H^{p}\left(X, \mathfrak{L}\left(m \chi_{o}\right)\right)=\varliminf_{\mathfrak{w} \in \overleftarrow{W} / W} H^{p}\left(X_{\mathfrak{w}}, \mathfrak{L}\left(m \chi_{o}\right)_{\left.\right|_{X_{\mathfrak{w}}}}\right) .
$$

Since any $g \in \widetilde{\mathcal{G}}$ acts as an algebraic automorphism of the line bundle $\mathfrak{L}\left(m \chi_{o}\right)$ (cf. $\S$ C.6), $H^{p}\left(X, \mathfrak{L}\left(m \chi_{o}\right)\right)$ is canonically a $\tilde{\mathcal{G}}$-module. This module is determined in $\left[\mathrm{Ku}_{1}\right]$ ( and also in $[\mathrm{M}]$ ). We summarize the results :

$$
\begin{gathered}
H^{p}\left(X, \mathfrak{L}\left(m \chi_{o}\right)\right)=0, \text { if } p>0 \text { and } m \geq 0, \\
H^{0}\left(X, \mathfrak{L}\left(m \chi_{o}\right)\right)=0, \text { if } m<0, \text { and } \\
H^{0}\left(X, \mathfrak{L}\left(m \chi_{o}\right)\right) \simeq L(\mathbb{C}, m)^{*} \text { for } m \geq 0, \text { as } \widetilde{\mathcal{G}}-\text { modules }
\end{gathered}
$$

where $L(\mathbb{C}, m)$ is the integrable highest weight (irreducible) $\tilde{\mathfrak{g}}$-module corresponding to the trivial $\mathfrak{g}$-module $\mathbb{C}$ and the central charge $m$ (cf. $\S \mathrm{A} .2)$, and $L(\mathbb{C}, m)^{*}$ denotes its full vector space dual. (By $\S$ C. $4, L(\mathbb{C}, m)$ and hence $L(\mathbb{C}, m)^{*}$ acquires a canonical structure of $\widetilde{\mathcal{G}}$-module.) For any subgroup $H \subset \widetilde{\mathcal{G}}$, by $H^{p}\left(X, \mathfrak{L}\left(m \chi_{o}\right)\right)^{H}$ we mean the space of $H$-invariants in $H^{p}\left(X, \mathfrak{L}\left(m \chi_{o}\right)\right)$.

Recall the definition of the map $\varphi: \mathcal{G} \rightarrow \mathcal{X}_{o}$ from $\S 1.4$, and the family $\mathcal{U}$ parametrized by $X$ from Proposition (2.8). Now define

$$
\begin{aligned}
X^{s} & =\{g \mathcal{P} \in X: \varphi(g) \text { is semistable }\} \\
& =\left\{x \in X: \mathcal{U}_{\mid C \times x} \text { is semistable }\right\}
\end{aligned}
$$

and set (for any $\mathfrak{w} \in \widetilde{W} / W$ ) 
Then by $\left[\mathrm{R}_{1}\right.$, Proposition (4.8)], $X_{\mathfrak{w}}^{s}$ is a Zariski open (and non-empty, since $1 \in$ $X_{\mathfrak{w}}^{s}$ ) subset of $X_{\mathfrak{w}}$, in particular, $X^{s}$ is a Zariski open subset of $X$. Now define

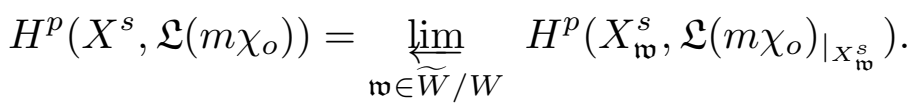

Clearly $\Gamma$ keeps $X^{s}$ stable, and by $\S 2.7, \Gamma$ acts morphically on the line bundle $\mathfrak{L}\left(m \chi_{o}\right)$ for any $m$ which is a multiple of $m_{V}$ (for some finite dimensional representation $V$ of $G)$, in particular, $\Gamma$ acts on the cohomology $H^{p}\left(X^{s}, \mathfrak{L}\left(m \chi_{o}\right)\right)$, and we can talk of the space of $\Gamma$-invariants $H^{p}\left(X^{s}, \mathfrak{L}\left(m \chi_{o}\right)\right)^{\Gamma}$.

The family $\mathcal{U}_{\mid X^{s}}$ yields a morphism $\psi: X^{s} \rightarrow \mathfrak{M}$, which maps any $x \in X^{s}$ to the $S$-equivalence class of the semistable bundle $\mathcal{U}_{x}$, where $\mathfrak{M}$ is the moduli space of semistable $G$-bundles on $C$ (cf. Theorem 3.4).

(6.2) Lemma. There exists a $v_{o} \in \widetilde{W} / W$ such that

$$
\psi\left(X_{\mathfrak{v}_{o}}^{s}\right)=\mathfrak{M} \text {. }
$$

Proof. Since $\bigcup_{\mathfrak{w}} X_{\mathfrak{w}}^{s}=X^{s}$ and $\psi\left(X^{s}\right)=\mathfrak{M}$, we get that $\mathfrak{M}=\bigcup_{\mathfrak{w}} \psi\left(X_{\mathfrak{w}}^{s}\right)$. But by a result of Chevalley (cf. [B, Chapter AG, Corollary 10.2]), $\psi\left(X_{\mathfrak{w}}^{s}\right)$ is a finite union of locally closed subvarieties $\left\{\mathfrak{M}_{\mathfrak{w}}^{i}\right\}$ of $\mathfrak{M}$. Hence $\mathfrak{M}$ is a countable union $\bigcup \mathfrak{M}_{\mathfrak{w}}^{i}$ of locally closed subvarieties. But then, by a Baire category argument, $\mathfrak{M}$ is a certain finite union of (locally closed) subvarieties $\left\{\mathfrak{M}_{\mathfrak{w}_{1}}^{1}, \ldots, \mathfrak{M}_{\mathfrak{w}_{n}}^{n}\right\}$. Now choosing a $\mathfrak{v}_{o} \in \widetilde{W} / W$ such that $\mathfrak{v}_{o} \geq \mathfrak{w}_{i}$, for all $1 \leq i \leq n$, we get that $\mathfrak{M}=\psi\left(X_{\mathfrak{v}_{o}}^{s}\right)$. This proves the lemma.

(6.3) Corollary. The moduli space $\mathfrak{M}$ is a unirational variety.

Proof. Since $X_{\mathfrak{w}}^{s}$ is an open subset of $X_{\mathfrak{w}}$ and $X_{\mathfrak{w}}$ is a rational variety ( by the Bruhat decomposition), the corollary follows from the above lemmma (6.2).

(6.4) Proposition. For any $d \geq 0$ and any finite dimensional representation $V$ of $G$, the canonical map

$$
\psi^{*}: H^{0}\left(\mathfrak{M}, \Theta(V)^{\otimes d}\right) \rightarrow H^{0}\left(X^{s}, \psi^{*}(\Theta(V))^{\otimes d}\right)^{\Gamma}
$$

is an isomorphism, where $\Theta(V)$ is the theta bundle on the moduli space $\mathfrak{M}$ associated to the representation $V$ (cf. §3.7), and the vector space on the right denotes the space of $\Gamma$-invariants under its natural action on the line bundle $\psi^{*}(\Theta(V))$. (Since the map $\psi: X^{s} \rightarrow \mathfrak{M}$ is $\Gamma$-equivariant, with trivial action of $\Gamma$ on $\mathfrak{M}$, the pull-back bundle $\psi^{*}(\Theta(V))$ has a natural $\Gamma$-action.)

Proof. Using Lemma (6.2) we see that the map $\psi^{*}$ is injective. Now the second part of Proposition (2.8), and Proposition (3.6) show that if $x$ and $y$ are two points in $X^{s}$ with $\mathcal{U}_{y} \simeq \operatorname{gr}\left(\mathcal{U}_{x}\right)$, then $y$ belongs to the Zariski closure of the $\Gamma$-orbit of $x$. In particular, two points in $X^{s}$ are in the same fiber of $\psi$ if and only if the closures of their $\Gamma$-orbits intersect. This, in turn, shows that if $\sigma$ is a $\Gamma$-invariant regular section of $\psi^{*}(\Theta(V))^{\otimes d}$ on $X^{s}$, it is induced from a set theoretic section $\underline{\sigma}$ of $\Theta(V)^{\otimes d}$ on $\mathfrak{M}$. That $\underline{\sigma}$ is regular, is seen by taking any Schubert variety $X_{\mathfrak{w}}$ such that $\psi\left(X_{\mathfrak{w}}^{s}\right)=\mathfrak{M}$ (cf. Lemma 6.2) and applying Proposition (4.1) to the morphism $\psi_{\mid X_{\mathfrak{w}}^{s}}: X_{\mathfrak{w}}^{s} \rightarrow \mathfrak{M}$.

By the functorial property of the theta bundle, $\Theta(\mathcal{U}(V))_{\mid X^{s}}$ is canonically isomorphic to $\psi^{*}(\Theta(V))$, since $\psi$ is defined using the restriction of the family $\mathcal{U}(V)$ to $X^{s}$ (cf. $\left.\S 3.7\right)$. Moreover, as observed in $\S 3.7$, the line bundles $\Theta(\mathcal{U}(V))$ and 
(6.5) Proposition. Any $\Gamma$-invariant regular section of $\psi^{*}(\Theta(V))^{\otimes d}$ on $X^{s}$ extends uniquely to a regular section of $(\operatorname{Det} \mathcal{U}(V))^{\otimes d}$ on $X$.

This proposition will be proved in the next section.

We now state and prove our main theorem, assuming the validity of Proposition (6.5).

(6.6) Theorem. Let the triple $\mathfrak{T}=(G, C, p)$ be as in $\S 1.1$, and let $V$ be a finite dimensional representation of $G$. Then, for any $d \geq 0$,

$$
H^{0}\left(\mathfrak{M}, \Theta(V)^{\otimes d}\right) \simeq H^{0}\left(\mathcal{G} / \mathcal{P}, \mathfrak{L}\left(d m_{V} \chi_{o}\right)\right)^{\Gamma}
$$

where the latter space of $\Gamma$-invariants is defined in $\S 6.1$, the integer $m_{V}$ is the Dynkin index of $V$ defined in $\S 5.1$, and the moduli space $\mathfrak{M}$ and the theta bundle $\Theta(V)$ are as in $\S \S 3.4$ and 3.7 respectively.

In particular, $H^{0}\left(\mathcal{G} / \mathcal{P}, \mathfrak{L}\left(d m_{V} \chi_{o}\right)\right)^{\Gamma}$ is finite dimensional.

(Observe that by (4) of $\S 6.1, H^{0}\left(\mathcal{G} / \mathcal{P}, \mathfrak{L}\left(d m_{V} \chi_{o}\right)\right)^{\Gamma}$ is isomorphic with the space of $\Gamma$-invariants in the dual space $L\left(\mathbb{C}, d m_{V}\right)^{*}$.)

Proof. We first begin with some simple observations:

(a) For any algebraic line bundle $\mathfrak{L}$ on $X$, the canonical restriction map $H^{0}(X, \mathfrak{L}) \rightarrow$ $H^{0}\left(X^{s}, \mathfrak{L}_{\mid X^{s}}\right)$ is injective: This is seen by restricting a section to each Schubert variety $X_{\mathfrak{w}}$, and observing that $X_{\mathfrak{w}}^{s}$ is non-empty, and open (and hence dense) in the irreducible variety $X_{\mathfrak{w}}$.

(b) If $\mathfrak{L}$ is a $\Gamma$-equivariant line bundle on $X$ (with respect to the standard action of $\Gamma$ on $X)(c f . \S B .7)$ and $\sigma$ is a regular section of $\mathfrak{L}$ such that its restriction to $X^{s}$ is $\Gamma$-invariant, then $\sigma$ itself is $\Gamma$-invariant: By $\Gamma$-invariance, for $\gamma \in \Gamma$, the section $\gamma^{*}(\sigma)-\sigma$ vanishes on $X^{s}$ (and hence on the whole of $X$ ).

(c) Suppose that $\mathfrak{L}^{\prime}$ and $\mathfrak{L}^{\prime \prime}$ are two $\Gamma$-equivariant line bundles on $X^{s}$. Then any biregular isomorphism of line bundles $\xi: \mathfrak{L}^{\prime} \rightarrow \mathfrak{L}^{\prime \prime}$ (inducing the identity on the base) in fact is $\Gamma$-equivariant. In particular, $\xi$ induces an isomorphism of the corresponding spaces of $\Gamma$-invariant regular sections:

Define a map $\epsilon: \Gamma \times X^{s} \rightarrow \mathbb{C}^{*}$ by

$$
\epsilon(\gamma, x)=L_{\gamma^{-1}} \xi_{\gamma x} L_{\gamma}\left(\xi_{x}\right)^{-1} \in \operatorname{Aut}_{\mathbb{C}}\left(\mathfrak{L}_{x}^{\prime \prime}\right)=\mathbb{C}^{*},
$$

for $\gamma \in \Gamma$ and $x \in X^{s}$, where $L_{\gamma}$ is the action of $\gamma$ on the appropriate line bundles, and $\xi_{x}$ denotes the restriction of $\xi$ to the fiber over $x \in X^{s}$. It is easy to see that $\epsilon$ is a regular map, and of course $\epsilon(1, x)=1$ for all $x \in X^{s}$. In particular, by Corollary (2.4), $\epsilon(\gamma, x)=1$, for all $\gamma \in \Gamma$. This proves assertion (c).

We now consider $(\operatorname{Det} \mathcal{U}(V))_{\left.\right|^{s}}^{\otimes d}$ as a $\Gamma$-equivariant line bundle by transporting the natural $\Gamma$-action on $\psi^{*}(\Theta(V))^{\otimes d}$ (cf. Proposition 6.4), via the canonical identification

$$
\operatorname{Det} \mathcal{U}(V)_{\mid X^{s}} \simeq \psi^{*}(\Theta(V)) \text {. }
$$

Choose an isomorphism of line bundles on $X$ 
which exists by Theorem (5.4). Recall from $\S 2.7$ that $\mathfrak{L}\left(\chi_{o}\right)^{\otimes d m_{V}}$ is a $\Gamma$-equivariant line bundle on $X$. Hence by (c) above, the map $\xi:=\xi_{o \mid X^{s}}$ is automatically $\Gamma$ equivariant. We have the following commutative diagram:

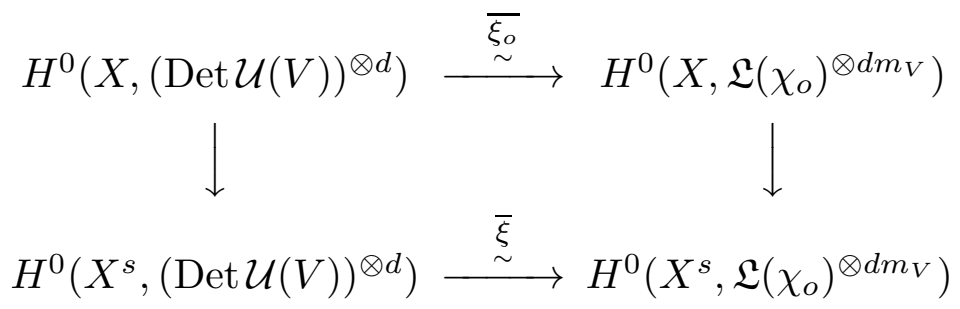

where $\bar{\xi}$ (resp. $\overline{\xi_{o}}$ ) is induced from $\xi$ (resp. $\xi_{o}$ ), and the vertical maps are the canonical restriction maps. Observe that $\bar{\xi}$ is $\Gamma$-equivariant (since $\xi$ is so).

Further we have

$$
\begin{aligned}
H^{0}\left(\mathfrak{M}, \Theta(V)^{\otimes d}\right) & \simeq H^{0}\left(X^{s},(\operatorname{Det} \mathcal{U}(V))^{\otimes d}\right)^{\Gamma} \quad(\text { by }(1) \text { and Proposition } 6.4) \\
& \simeq H^{0}\left(X^{s}, \mathfrak{L}\left(\chi_{o}\right)^{\otimes d m_{V}}\right)^{\Gamma} \quad(\text { under } \bar{\xi})
\end{aligned}
$$

We complete the proof of the theorem by showing that the restriction map

$$
H^{0}\left(X, \mathfrak{L}\left(\chi_{o}\right)^{\otimes d m_{V}}\right)^{\Gamma} \rightarrow H^{0}\left(X^{s}, \mathfrak{L}\left(\chi_{o}\right)^{\otimes d m_{V}}\right)^{\Gamma}
$$

is an isomorphism:

It suffices to show that any $\Gamma$ - invariant section $\sigma$ of $\mathfrak{L}\left(\chi_{o}\right)^{\otimes d m_{V}}$ over $X^{s}$ extends to a section over $X$, for then the extension will automatically be $\Gamma$-invariant by (b) and unique by (a). By the above commutative diagram, this is equivalent to showing that any $\Gamma$-invariant section $\sigma_{o}$ of $(\operatorname{Det} \mathcal{U}(V))^{\otimes d}$ over $X^{s}$ extends to the whole of $X$. But this is the content of Proposition (6.5), thereby completing the proof of the theorem.

(6.7) Proposition. For any $d \geq 0$, and finite dimensional representation $V$ of $G$, we have

$$
\left[L\left(\mathbb{C}, d m_{V}\right)^{*}\right]^{\Gamma}=\left[L\left(\mathbb{C}, d m_{V}\right)^{*}\right]^{\operatorname{Lie} \Gamma}=\left[L\left(\mathbb{C}, d m_{V}\right)^{*}\right]^{\mathfrak{g} \otimes k\left[C^{*}\right]},
$$

where $L\left(\mathbb{C}, d m_{V}\right)$ is canonically an algebraic $\Gamma$-module as in $\S 2.7, \mathfrak{g}$ is the Lie algebra of the group $G$ and (as in $\S 1.1$ ) $k\left[C^{*}\right]$ is the ring of regular functions on the affine curve $C^{*}$.

Proof. Abbreviate $L\left(\mathbb{C}, d m_{V}\right)$ by $V$. Fix $v \in V$ and consider the morphism $\pi_{v}$ : $\Gamma \rightarrow V$ given by $\pi_{v}(\gamma)=\gamma \cdot v$ for $\gamma \in \Gamma$. Recall (cf. Lemma B.6) that, by definition, the action of the Lie algebra Lie $\Gamma$ on $v \in V$ is given by the induced map $\left(d \pi_{v}\right)_{e}$ : $T_{e}(\Gamma)=$ Lie $\Gamma \rightarrow T_{v}(V)=V$.

Fix $\theta \in V^{*}$. For any $v \in V$, define the map $\theta_{v}: \Gamma \rightarrow \mathbb{A}^{1}$ by $\theta_{v}(\gamma)=\theta(\gamma \cdot v)$. The induced map $\left(d \theta_{v}\right)_{e}: T_{e}(\Gamma)=$ Lie $\Gamma \rightarrow T_{\theta(v)}\left(\mathbb{A}^{1}\right)=\mathbb{A}^{1}$ is given by

$$
\left(d \theta_{v}\right)_{e}(a)=\theta(a \cdot v), \text { for } a \in \operatorname{Lie} \Gamma .
$$

For any $\gamma_{o} \in \Gamma$, we now determine the map $\left(d \theta_{v}\right)_{\gamma_{o}}$ : Consider the right translation map $R_{\gamma_{o}}: \Gamma \rightarrow \Gamma$, given by $R_{\gamma_{o}}(\gamma)=\gamma \gamma_{o}$. Then we have 
If $\theta \in\left[V^{*}\right]^{\Gamma}$, then $\theta_{v}$ (for any fixed $v \in V$ ) is the constant map $\gamma \mapsto \theta(v)$. In particular, $\left(d \theta_{v}\right)_{e} \equiv 0$, proving (by 1) that $\theta \in\left[V^{*}\right]^{\operatorname{Lie} \Gamma}$. Conversely, take $\theta \in\left[V^{*}\right]^{\operatorname{Lie} \Gamma}$. Then by (1) and (2), for any fixed $v \in V,\left(d \theta_{v}\right)_{\gamma_{o}} \equiv 0$ for any $\gamma_{o} \in \Gamma$. In particular, for any fixed $v \in V$ and $i \geq 0$, the map $\theta_{v_{\mid \Gamma_{i}}}: \Gamma_{i} \rightarrow \mathbb{A}^{1}\left(\Gamma_{i}\right.$ is as in proof of Lemma 2.1) is constant on the irreducible components of $\Gamma_{i}$ (as the base field is of char. 0). But since $\Gamma$ is connected (cf. proof of Corollary 2.4), $\theta_{v}$ itself is forced to be a constant. Thus, we have $(\gamma \theta-\theta) v=0$, for every $v \in V$ and $\gamma \in \Gamma$; proving that $\theta \in\left[V^{*}\right]^{\Gamma}$. Finally, by Lemma (2.2), we have Lie $\Gamma=\mathfrak{g} \otimes k\left[C^{*}\right]$. This proves the proposition.

(6.8) Remarks. (a) From the proof it is clear that the above proposition is true with $L\left(\mathbb{C}, d m_{V}\right)$ replaced by any algebraic representation of the algebraic group $\Gamma$.

(b) In Conformal Field Theory, the space of vacua is defined to be the space of invariants $\left[L(\mathbb{C}, d)^{*}\right]^{\mathfrak{g} \otimes k\left[C^{*}\right]}$ of the Lie algebra $\mathfrak{g} \otimes k\left[C^{*}\right]$ (cf. [TUY, Definition 2.2.2]). We see, by Theorem (6.6) and Proposition (6.7), that the space of vacua is isomorphic to the space of generalised theta functions.

(c) Assertion (c) in the proof of Theorem (6.6) is true with $X^{s}$ replaced by $X$. (In fact, in this case we do not even need to use Corollary (2.4), but need only the connectedness of $\Gamma$.) We outline an argument:

Following the same notation as in the proof of assertion (c), in this case, for any fixed $\gamma \in \Gamma$ the map $\epsilon_{\left.\right|_{\gamma \times X}}: \gamma \times X \rightarrow \mathbb{C}^{*}$ is a constant $\alpha_{\gamma}$ (since $X$ is a connected projective ind-variety). From this it can been easily seen that the map $\alpha: \Gamma \rightarrow \mathbb{C}^{*}$ taking $\gamma \mapsto \alpha_{\gamma}$ is a group morphism. In particular, the derivative $d \alpha$ : Lie $\Gamma \rightarrow \mathbb{C}$ is a Lie algebra homomorphism. Since the commutator $[\operatorname{Lie} \Gamma$, Lie $\Gamma]=\operatorname{Lie} \Gamma$, we get that $d \alpha \equiv 0$. Hence, by an argument used in the proof of Proposition (6.7), we see that the map $\alpha$ itself is identically 1. This proves assertion (c) for $X$.

Now, as shown in $[\mathrm{KN}]$, the algebraic $\Gamma$-action on the line bundle $(\operatorname{Det} \mathcal{U}(V))_{\left.\right|_{X_{s}}}^{\otimes d}$ can be extended to an algebraic $\Gamma$-action on the line bundle $(\operatorname{Det} \mathcal{U}(V))^{\otimes d} \rightarrow X$. In particular, the isomorphism $\xi_{0}:(\operatorname{Det} \mathcal{U}(V))^{\otimes d} \rightarrow \mathcal{L}\left(\chi_{0}\right)^{\otimes d m_{V}}$ (cf. proof of Theorem $6.6)$ is $\Gamma$-equivariant and hence so is its restriction to $X_{s}$. This provides a proof of

Theorem (6.6), without using Corollary 2.4 (but using only the connectedness of $\Gamma)$.

As an immediate consequence of the above remark (b), we obtain the following.

(6.9) Corollary. Let the notation and assumptions be as in Theorem (6.6). Then the space of covariants $L\left(\mathbb{C}, d m_{V}\right) /\left(\left(\mathfrak{g} \otimes_{k} k\left[C^{*}\right]\right) . L\left(\mathbb{C}, d m_{V}\right)\right)$ is finite dimensional. (Cf. [K, Exercise 11.10, p. 209] for a purely algebraic proof of this corollary.)

\section{Proof of Proposition (6.5).}

(7.1) Lemma. Let $X$ be an irreducible normal variety, $U \subset X$ a non-empty open subset and $\mathfrak{L}$ a line bundle on $X$. Then any element of $\underset{n \in \mathbb{Z}_{+}}{\oplus} H^{0}\left(U, \mathfrak{L}^{n}\right)$ which is intearal over $\oplus H^{0}\left(X, \mathfrak{L}^{n}\right)$ belonas to $\oplus H^{0}\left(X, \mathfrak{L}^{n}\right)$. 
Proof. Since the rings in question are graded, it suffices to prove the lemma only for homogeneous elements. Let $b \in H^{0}\left(U, \mathfrak{L}^{n_{o}}\right)$ be integral over $\oplus H^{0}\left(X, \mathfrak{L}^{n}\right)$, i.e., $b$ satisfies a relation $b^{m}+a_{1} b^{m-1}+\cdots+a_{m}=0$ with $a_{i} \in \oplus H^{0}\left(X, \mathfrak{L}^{n}\right)$. Let $D$ be a prime divisor in $X \backslash U$ and let $b$ have a pole of order $\ell \geq 0$ along $D$. Then the order of the pole of $b^{m}$ along $D$ is of course $\ell m$ and that of $a_{i} b^{m-i}$ is $\leq \ell(m-1)$ for every $i \geq 1$. But since $b^{m}+a_{1} b^{m-1}+\cdots+a_{m-1} b$ is by assumption regular along $D$, we are forced to have $\ell=0$, i.e., $b$ is regular along $D$. Hence $b \in H^{0}\left(X, \mathfrak{L}^{n_{o}}\right)$.

(7.2) Lemma. Let $f: X \rightarrow Y$ be a morphism between projective varieties and $\mathcal{L}$ an ample line bundle on $Y$. Then the ring $\underset{n \geq 0}{\oplus} H^{0}\left(X, f^{*} \mathcal{L}^{n}\right)$ is integral over the ring $\underset{n \geq 0}{\oplus} H^{0}\left(Y, \mathcal{L}^{n}\right)$.

Proof. First of all,

$$
\underset{n \geq 0}{\oplus} H^{0}\left(X, f^{*} \mathcal{L}^{n}\right) \approx H^{0}\left(Y, \mathcal{L}^{n} \otimes_{\mathcal{O}} f_{*} \mathcal{O}\right)
$$

Since $f_{*} \mathcal{O}$ is coherent, we can write

$$
0 \rightarrow \mathcal{K} \rightarrow \mathcal{L}_{1} \oplus \cdots \oplus \mathcal{L}_{m} \rightarrow f_{*} \mathcal{O} \rightarrow 0
$$

for some line bundles $\mathcal{L}_{i}$ on $Y$. Since $\mathcal{L}$ is ample on $Y$, there exists $n_{o} \geq 1$ such that for $n \geq n_{o}, H^{1}\left(Y, \mathcal{L}^{n} \otimes \mathcal{K}\right)=0$; in particular,

$$
H^{0}\left(Y, \mathcal{L}^{n} \underset{\mathcal{O}}{\otimes}\left(\underset{i}{\oplus} \mathcal{L}_{i}\right)\right) \rightarrow H^{0}\left(Y, \mathcal{L}^{n} \underset{\mathcal{O}}{\otimes} f_{*} \mathcal{O}\right)
$$

is surjective for $n \geq n_{o}$. We now prove that $\underset{n \geq 0}{\oplus} H^{0}\left(Y, \mathcal{L}^{n} \otimes \mathcal{L}_{i}\right)$ is finitely generated over $\underset{n \geq 0}{\oplus} H^{0}\left(Y, \mathcal{L}^{n}\right)$, for any line bundle $\mathcal{L}_{i}$ on $Y$ :

Consider the sheaf exact sequence (where $\Delta(Y) \subset Y \times Y$ is the diagonal)

$$
0 \rightarrow \mathcal{I}_{\Delta(Y)} \rightarrow \mathcal{O}_{Y \times Y} \rightarrow \mathcal{O}_{Y} \rightarrow 0
$$

There exist $\ell_{0}, m_{0} \geq 1$ such that for $n \geq \ell_{0}$ and $m \geq m_{0}, H^{1}\left(Y \times Y, \mathcal{I}_{\Delta(Y)} \otimes\left(\mathcal{L}^{n} \nabla\right.\right.$ $\left.\left.\left(\mathcal{L}^{m} \otimes \mathcal{L}_{i}\right)\right)\right)=0$. In particular, for $n \geq \ell_{0}$,

$$
H^{0}\left(Y, \mathcal{L}^{n}\right) \otimes H^{0}\left(Y, \mathcal{L}^{m_{0}} \otimes \mathcal{L}_{i}\right) \rightarrow H^{0}\left(Y, \mathcal{L}^{n+m_{0}} \otimes \mathcal{L}_{i}\right)
$$

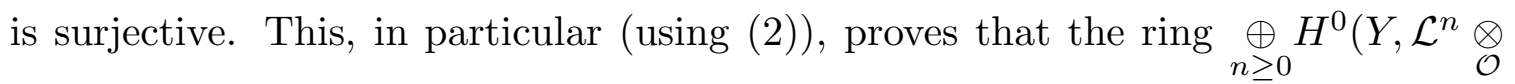
$\left.f_{*}(\mathcal{O})\right)$ is finitely generated over $\underset{n \geq 0}{\oplus} H^{0}\left(Y, \mathcal{L}^{n}\right)$, in particular, is integral (cf. [AM]). This proves the lemma by (1).

(7.3) Proposition. Let $Y$ be a normal irreducible variety parametrizing a family $\mathcal{E}$ of $G$-bundles. Consider the induced map $\beta: Y^{s} \rightarrow \mathfrak{M}$ (cf. Theorem 3.4; where $Y^{s}$ is the subset of $Y$ consisting of those $y \in Y$ such that $\mathcal{E}_{y}$ is semistable). Fix a representation $V$ of $G$ and fix an isomorphism 
Then for any section $\sigma \in H^{0}\left(\mathfrak{M}, \Theta(V)^{\otimes d}\right)(d \geq 0)$, the pull-back section $\beta^{*} \sigma$ extends to an element of $H^{0}\left(Y, \Theta(\mathcal{E}(V))^{\otimes d}\right)$.

Proof. Consider the diagram (cf. §3.7)

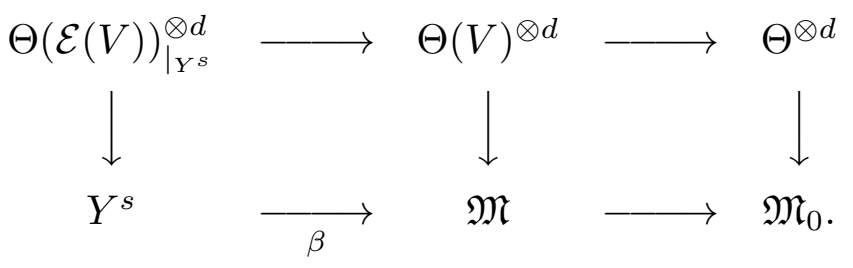

By Lemma (7.1), it suffices to show that $\beta^{*} \sigma \in H^{0}\left(Y^{s}, \Theta(\mathcal{E}(V))^{\otimes d}\right)$ is integral over $\underset{n \geq 0}{\oplus} H^{0}\left(Y, \Theta(\mathcal{E}(V))^{\otimes n}\right)$ :

Since $\sigma$ is integral over $\underset{n \geq 0}{\Theta} H^{0}\left(\mathfrak{M}_{o}, \Theta^{\otimes n}\right)$ (by Lemma 7.2), there exists a relation

$$
\sigma^{p}+a_{1} \sigma^{p-1}+\cdots+a_{p}=0, \quad \text { for some } a_{i} \in \underset{n \geq 0}{\oplus} H^{0}\left(\mathfrak{M}_{o}, \Theta^{\otimes n}\right) .
$$

Now we have

$$
\begin{array}{llll}
Y^{s} & \hookrightarrow & Y_{o}^{s} \\
\beta \downarrow & & \downarrow \beta_{o} \\
\mathfrak{M} & \rightarrow & \mathfrak{M}_{o}
\end{array}
$$

where $Y_{o}^{s}:=\left\{y \in Y: \mathcal{E}_{y}(V)\right.$ is a semistable $G L(V)$-bundle $\}$. Assume that $\beta_{o}^{*} a_{i}$ can be extended to the whole of $Y$ (which is possible by the following Lemma 7.5), then we get from $(*)$

$$
\left(\beta^{*} \sigma\right)^{p}+\left(\beta_{0}^{*} a_{1}\right)_{Y_{Y^{s}}}\left(\beta^{*} \sigma\right)^{p-1}+\cdots+\left(\beta_{0}^{*} a_{p}\right)_{\left.\right|_{Y^{s}}}=0
$$

which proves the proposition.

(7.4) G.I.T. construction of the moduli of vector bundles. We recall the construction of the moduli space of vector bundles on $C$ using G.I.T.. Let $r \geq 1$ and $\delta$ be integers. For the fixed point $p \in C$ and for a coherent sheaf $\mathbf{F}$ on $C$, put $\mathbf{F}(m)=\mathbf{F} \otimes_{\mathcal{O}} \mathcal{O}(m p)$, for any $m \in \mathbb{Z}$, where $\mathcal{O}=\mathcal{O}_{C}$ is the structure sheaf of $C$. We can choose a large enough integer $m_{o}=m_{o}(r, \delta)$ such that for any semistable vector bundle $\mathbf{E}$ of rank $r$ and degree $\delta$ on $C$, we have $H^{1}\left(\mathbf{E}\left(m_{o}\right)\right)=0$ and $\mathbf{E}\left(m_{o}\right)$ is generated by its global sections. Let $q=\operatorname{dim} H^{0}\left(\mathbf{E}\left(m_{o}\right)\right)=\delta+r\left(m_{o}+1-g\right)$ (where $g$ is the genus of $C$ ) and consider the Grothendieck quot scheme $Q$ consisting of coherent sheaves on $C$ which are quotients of $\mathbb{C}^{q} \otimes_{\mathbb{C}} \mathcal{O}$ with Hilbert polynomial (in the indeterminate $v) r v+q$. The group $G L(q, \mathbb{C})$ operates canonically on $Q$ and the action on $C \times Q$ (with the trivial action on $C$ ) lifts to an action of the tautological sheaf $\mathcal{T}$ on $C \times Q$.

We denote by $R_{o}$ the $G L(q)$-invariant open subset of $Q$ consisting of those $x \in Q$ such that $\mathcal{T}_{x}=\mathcal{T}_{\mid C \times x}$ is locally free and such that the following canonical map is an isomorphism:

$$
\mathbb{C}^{q}=H^{0}\left(\mathbb{C}^{q} \otimes_{\mathbb{C}} \mathcal{O}\right) \stackrel{\sim}{\rightarrow} H^{0}\left(\mathcal{T}_{x}\right) .
$$

Then $R_{o}$ is smooth and irreducible and $\mathcal{T}_{\mid C \times R_{o}}$ is a rank- $r$ vector bundle. Define 
large $m_{o}$, the G.I.T. quotient $R_{o}^{s} / / G L(q)$ yields the moduli space $\mathfrak{M}_{o}$ of vector bundles of rank $r$ and degree $\delta$. (We choose such a $m_{o}$ in the sequel.) (For all this, see [NRa, Appendix A] or [Le].)

Now let $\mathcal{V}_{o} \rightarrow C \times T_{o}$ be a family of vector bundles of rank $r$ and degree $\delta$ (parametrized by a variety $T_{o}$ ). We can find an integer $m_{T_{o}}$ such that for $m \geq m_{T_{o}}$, we have :

(1) $R^{1} p_{2_{*}}\left(\mathcal{V}_{o}(m)\right)=0$

(2) $p_{2_{*}}\left(\mathcal{V}_{o}(m)\right)$ is a vector bundle on $T_{o}$ ( of rank $q:=\delta+r(m+1-g)$ ), and

(3) the canonical map $p_{2}^{*} p_{2_{*}}\left(\mathcal{V}_{o}(m)\right) \rightarrow \mathcal{V}_{o}(m)$ is surjective,

where $p_{2}: C \times T_{o} \rightarrow T_{o}$ (resp. $p_{1}: C \times T_{o} \rightarrow C$ ) is the projection on the second (resp first) factor, and $\mathcal{V}_{o}(m):=\mathcal{V}_{o} \otimes_{\mathcal{O}_{C \times T_{o}}} p_{1}^{*} \mathcal{O}(m p)$.

Choose $\bar{m}_{o}$ larger than $m_{T_{o}}$ and $m_{o}$, where $m_{o}$ is as above. Let $\mathbf{P}_{o}$ be the frame bundle of $p_{2_{*}}\left(\mathcal{V}_{o}\left(\bar{m}_{o}\right)\right)$ with the projection $\pi_{o}: \mathbf{P}_{o} \rightarrow T_{o}$. Then there exists a canonical $G L(q)$-equivariant morphism $\varphi_{o}: \mathbf{P}_{o} \rightarrow R_{o}$ such that the families $\pi_{o}^{*}\left(\mathcal{V}_{o}\right)$ and $\varphi_{o}^{*}\left(\mathcal{T}\left(-\bar{m}_{o}\right)\right)$ are isomorphic, where $\mathcal{T}(-m):=\mathcal{T} \otimes_{\mathcal{O}_{C \times R_{o}}} p_{C}^{*} \mathcal{O}(-m p)$ and $p_{C}: C \times R_{o} \rightarrow C$ is the projection on the first factor.

With the same notation as in Proposition (7.3) and its proof, we have the following:

(7.5) Lemma. For any $\sigma_{o} \in H^{0}\left(\mathfrak{M}_{o}, \Theta^{\otimes d}\right)(d \geq 0)$ the pull-back section $\beta_{o}^{*} \sigma_{o} \in$ $H^{0}\left(Y_{o}^{s}, \Theta(\mathcal{E}(V))^{\otimes d}\right)$ can be extended to the whole of $Y$.

Proof. In the construction as in $\S 7.4$, take $r=\operatorname{dim} V, \delta=0$, and $T_{o}=Y$.

Consider the diagram (where the map $\pi$ is the quotient map and the other maps are as explained in $\S \S 7.3-7.4)$ :

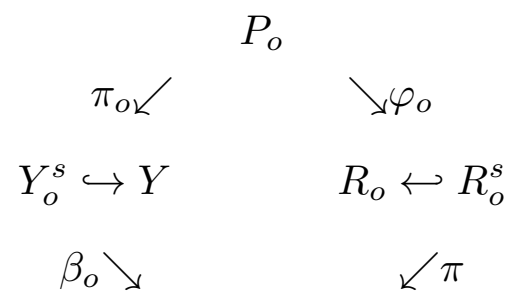

$\mathfrak{M}_{o}$

Now since $\operatorname{codim}_{R_{o}}\left(R_{o} \backslash R_{o}^{s}\right) \geq 2$ and $R_{o}$ is smooth (in particular normal), the section $\pi^{*} \sigma_{o}$ extends (say to $\overline{\pi^{*} \sigma_{o}}$ ) to the whole of $R_{o}$. Pull $\overline{\pi^{*} \sigma_{o}}$ via the GL $(q)$ equivariant morphism $\varphi_{o}$, and then push via the GL $(q)$-bundle $\pi_{o}$. This gives an extension of the section $\beta_{o}^{*} \sigma_{o}$ to the whole of $Y$. This proves the lemma, thereby proving Proposition (7.3) completely.

Finally we prove Proposition (6.5) and thus complete the proof of Theorem (6.6).

(7.6) Proof of Proposition (6.5). Let $\tilde{\sigma}$ be a $\Gamma$-invariant section of $\psi^{*}(\Theta(V))^{\otimes d}$ on $X^{s}$. By Proposition (6.4), there is a section $\sigma$ of $\Theta(V)^{\otimes d}$ over $\mathfrak{M}$ such that $\psi^{*}(\sigma)=\tilde{\sigma}$. Let $X_{\mathfrak{w}}$ be a Schubert variety. Since $X_{\mathfrak{w}}$ is irreducible and normal (cf. $\left[\mathrm{Ku}_{1}\right.$, Theorem 2.16]), by Proposition (7.3), $\tilde{\sigma}_{\mid X_{\mathrm{w}}^{s}}$ extends to a (unique) section $\bar{\sigma}_{\mathfrak{w}}$ of $(\operatorname{Det} \mathcal{U}(V))^{\otimes d}$ on $X_{\mathfrak{w}}$. By the uniqueness of extensions, it is clear that for 
of $(\text { Det } \mathcal{U}(V))^{\otimes d}$ on the whole of $X$, extending the section $\tilde{\sigma}$. This completes the proof of the proposition.

\section{Appendix A. Affine Lie algebras - Basic definitions and their represen- tations.}

The basic reference for this section is Kac's book $[\mathrm{K}]$.

(A.1) Definition. Let $\mathfrak{g}$ be a finite dimensional complex simple Lie algebra. (We also fix a Borel subalgebra $\mathfrak{b}$ and a Cartan subalgebra $\mathfrak{h} \subset \mathfrak{b}$ of $\mathfrak{g}$.) Then the associated affine Kac-Moody Lie algebra is by definition the space

$$
\tilde{\mathfrak{g}}:=\mathfrak{g} \otimes_{\mathbb{C}} \mathbb{C}\left[t^{ \pm 1}\right] \oplus \mathbb{C} K
$$

together with the Lie bracket (for $X, Y \in \mathfrak{g}$ and $P, Q \in \mathbb{C}\left[t^{ \pm 1}\right]$ )

$$
\begin{gathered}
{[X \otimes P, Y \otimes Q]=[X, Y] \otimes P Q+\left(<X, Y>\operatorname{Res}_{t=0}\left(\frac{d P}{d t} Q\right)\right) K, \text { and }} \\
{[\tilde{\mathfrak{g}}, K]=0,}
\end{gathered}
$$

where $\langle,>$ is the Killing term on $\mathfrak{g}$, normalized so that $\langle\theta, \theta\rangle=2$ for the highest root $\theta$ of $\mathfrak{g}$. We also define a certain completion $\tilde{\mathfrak{g}}_{\text {comp }}$ of $\tilde{\mathfrak{g}}$ by

$$
\tilde{\mathfrak{g}}_{\mathrm{comp}}=\mathfrak{g} \otimes_{\mathbb{C}} \mathbb{C}((t)) \oplus \mathbb{C} K
$$

where $\mathbb{C}((t))$ is the field of Laurent power series. Then $\tilde{\mathfrak{g}}_{\text {comp }}$ is a Lie algebra under the same bracket as above.

The Lie algebra $\mathfrak{g}$ sits as a Lie subalgebra of $\tilde{\mathfrak{g}}$ as $\mathfrak{g} \otimes t^{0}$. The Lie algebra $\tilde{\mathfrak{g}}$ admits a distinguished 'parabolic' subalgebra

$$
\tilde{\mathfrak{p}}:=\mathfrak{g} \otimes \mathbb{C}[t] \oplus \mathbb{C} K
$$

We also define its 'nil-radical' $\tilde{\mathfrak{u}}$ (which is an ideal of $\tilde{\mathfrak{p}}$ ) by

$$
\tilde{\mathfrak{u}}:=\mathfrak{g} \otimes t \mathbb{C}[t],
$$

and its 'Levi component' (which is a Lie subalgebra of $\tilde{\mathfrak{p}}$ )

$$
\tilde{\mathfrak{p}}^{o}:=\mathfrak{g} \otimes t^{o} \oplus \mathbb{C} K
$$

Clearly (as a vector space)

$$
\tilde{\mathfrak{p}}=\tilde{\mathfrak{u}} \oplus \tilde{\mathfrak{p}}^{o}
$$

Define the loop algebra $L(\mathfrak{g}):=\mathfrak{g} \otimes_{\mathbb{C}} \mathbb{C}\left[t^{ \pm 1}\right]$ with Lie bracket $[X \otimes P, Y \otimes Q]=$ $[X, Y] \otimes P Q$, for $X, Y \in \mathfrak{g}$ and $P, Q \in \mathbb{C}\left[t^{ \pm 1}\right]$. Then $\tilde{\mathfrak{g}}$ can be viewed as a onedimensional central extension of $L(\mathfrak{g})$ : 
where the Lie algebra homomorphism $\pi$ is defined by $\pi(X \otimes P)=X \otimes P$ and $\pi(K)=0$.

(A.2) Irreducible representations of $\tilde{\mathfrak{g}}$. Fix an irreducible (finite dimensional) representation $V$ of $\mathfrak{g}$ and a number $\ell \in \mathbb{C}$ (to be called the central charge). Then we define the associated generalized Verma module for $\tilde{\mathfrak{g}}$ as

$$
M(V, \ell)=U(\tilde{\mathfrak{g}}) \otimes_{U(\tilde{\mathfrak{p}})} I_{\ell}(V)
$$

where the $\tilde{\mathfrak{p}}$-module $I_{\ell}(V)$ has the underlying vector space same as $V$ on which $\tilde{\mathfrak{u}}$ acts trivially, the central element $K$ acts via the scalar $\ell$ and the action of $\mathfrak{g}=\mathfrak{g} \otimes t^{o}$ is via the $\mathfrak{g}$-module structure on $V$.

In the case when $\ell \neq-h$ (where $h$ is the dual Coxeter number of $\mathfrak{g}$; cf. Remark $5.3), M(V, \ell)$ has a unique irreducible quotient denoted $L(V, \ell)$. We assume in the sequel that $\ell \neq-h$. (It is easy to see that the $\tilde{\mathfrak{g}}$-module structure on $M(V, \ell)$ and $L(V, \ell)$ extends to a $\tilde{\mathfrak{g}}_{\text {comp }}$-module structure.)

(A.3) Remark. It is easy to see that any vector $v \in M(V, \ell)$ is contained in a finite dimensional $\mathfrak{g}$-submodule of $M(V, \ell)$. In particular, the same property holds for any vector in $L(V, \ell)$.

(A.4) Definition. Consider the Lie subalgebra $\mathfrak{r}^{o}$ of $\tilde{\mathfrak{g}}$ spanned by $\left\{Y_{\theta} \otimes t, \theta^{\vee} \otimes\right.$ $\left.1, X_{\theta} \otimes t^{-1}\right\}$, where $Y_{\theta}$ (resp. $X_{\theta}$ ) is a non-zero root vector of $\mathfrak{g}$ corresponding to the root $-\theta$ (resp. $\theta$ ) and the coroot $\theta^{\vee}$ is to be thought of as an element of $\mathfrak{h}$. Then the Lie algebra $\mathfrak{r}^{o}$ is isomorphic with $\operatorname{sl}(2)$ (cf. proof of Theorem 5.4).

A $\tilde{\mathfrak{g}}$-module $W$ is said to be integrable if every vector $v \in W$ is contained in a finite dimensional $\mathfrak{g}$-submodule of $W$ and also $v$ is contained in a finite dimensional $\mathfrak{r}^{o}$-submodule of $W$.

The following lemma follows as a consequence of $\operatorname{sl}(2)$-theory.

(A.5) Lemma. The irreducible module $L(V, \ell)$ (as in $\S$ A.2) is integrable if and only if $\ell$ is an integer and $\ell \geq\left\langle\lambda, \theta^{\vee}>\right.$, where $\lambda$ is the highest weight of $V$.

\section{Appendix B. An introduction to Ind-varieties.}

(B.1) Definitions $[\mathrm{Sa}]$. By an ind-variety we mean a set $X$ together with a filtration

$$
X_{o} \subseteq X_{1} \subseteq X_{2} \subseteq \ldots
$$

such that

(1) $\cup_{n \geq 0} X_{n}=X$, and

(2) Each $X_{n}$ is a (finite dimensional) variety over $k$ such that the inclusion $X_{n} \hookrightarrow X_{n+1}$ is a closed immersion.

An ind-variety $X$ is said to be projective (resp. affine) if each $X_{n}$ is projective (resp. affine). For an affine ind-variety $X$, we define its ring of regular functions $k[X]$ by $k[X]=\operatorname{Inv}_{n \rightarrow \infty} l_{\text {t. }} k\left[X_{n}\right]$. Putting the discrete topology on each $k\left[X_{n}\right]$ and taking the inverse limit topology on $k[X]$, we obtain $k[X]$ as a topological ring.

Let $X$ and $Y$ be two ind-varieties with filtrations $X_{n}$ and $Y_{n}$ respectively. A 
number $m(n) \geq 0$ such that $f\left(X_{n}\right) \subseteq Y_{m(n)}$ and moreover $f_{\mid X_{n}}: X_{n} \rightarrow Y_{m(n)}$ is a morphism. Clearly, a morphism $f: X \rightarrow Y$ (between two affine ind-varieties) induces a continuous $k$-algebra homomorphism $f^{*}: k[Y] \rightarrow k[X]$.

A morphism $f: X \rightarrow Y$ is said to be an isomorphism if $f$ is bijective and $f^{-1}: Y \rightarrow X$ also is a morphism. Two ind-variety structures on the same set $X$ are said to be equivalent if the identity map I: $X \rightarrow X$ is an isomorphism of ind-varieties.

We define the Zariski topology on an ind-variety $X$ by declaring a set $U \subseteq X$ open if and only if $U \cap X_{n}$ is Zariski open in $X_{n}$ for each $n$.

(B.2) Exercises. (a) For an ind-variety $X$ (under the Zariski topology), a subset $Z \subseteq X$ is closed if and only if $Z \cap X_{n}$ is closed in $X_{n}$ for each $n$.

(b) A morphism $f: X \rightarrow Y$ between two ind-varieties is continuous.

(c) Any continuous map $f: X \rightarrow Y$ between two ind-varieties satisfies that for each $n$, there exists a $m(n)$ such that $f\left(X_{n}\right) \subseteq Y_{m(n)}$.

(B.3) Examples. (1) Any (finite dimensional) variety $X$ is of course canonically an ind-variety, where we take each $X_{n}=X$.

(2) If $X$ and $Y$ are ind-varieties then $X \times Y$ is canonically an ind-variety, where we define the filtration by

$$
(X \times Y)_{n}=X_{n} \times Y_{n}
$$

(3) $\mathbb{A}^{\infty}:=\left\{\left(a_{1}, a_{2}, a_{3}, \cdots\right)\right.$; where all but finitely many $a_{i}^{\prime}$ s are zero and each $\left.a_{i} \in k\right\}$ is an ind-variety under the filtration : $\mathbb{A}^{1} \subset \mathbb{A}^{2} \subset \mathbb{A}^{3} \subset \cdots$, where $\mathbb{A}^{n} \subset \mathbb{A}^{\infty}$ is the set of all the sequences with $a_{n+1}=a_{n+2}=\cdots=0$, which of course is the $n$-dimensional affine space.

(4) Any vector space $V$ of countable dimension over $k$ is canonically an affine indvariety: Take a basis $\left\{e_{i}\right\}_{i \geq 1}$ of $V$. This gives rise to a $k$-linear isomorphism $\mathbb{A}^{\infty} \sim \vec{\rightarrow} V$ (taking $\left.\left(a_{1}, a_{2}, a_{3}, \cdots\right) \mapsto \sum a_{i} e_{i}\right)$. By transporting the ind-variety strucutre from $\mathbb{A}^{\infty}$ via this isomorphism, we get an (affine) ind-variety structure on $V$. It is easy to see that a different choice of basis of $V$ gives an equivalent ind-variety structure on $V$.

(B.4) Definitions. (a) Let $X$ be an ind-variety with the filtration $\left(X_{n}\right)$. For any $x \in X$, define the Zariski tangent space $T_{x}(X)$ of $X$ at $x$ by

$$
T_{x}(X)=\operatorname{limit}_{n \rightarrow \infty} T_{x}\left(X_{n}\right),
$$

where $T_{x}\left(X_{n}\right)$ is the Zariski tangent space of $X_{n}$ at $x$. (Observe that $x \in X_{n}$ for all large enough $n$.)

A morphism $f: X \rightarrow Y$ clearly induces a linear map $(d f)_{x}: T_{x}(X) \rightarrow T_{f(x)}(Y)$ (for any $x \in X$ ), called the derivative of $f$ at $x$.

(b) An ind-variety $H$ is said to an ind-algebraic group (for short an ind-group), if the underlying set $H$ is a group such that the map $H \times H \rightarrow H$, taking $(x, y) \mapsto$ $x y^{-1}$, is a morphism. In this article, we only have occasion to consider affine ind-groups, i.e., ind-algebraic groups $H$ such that $H$ is an affine ind-variety.

By a group morphism between two ind-groups $H$ and $K$, we mean a group 
An abstract representation of the ind-group $H$ in a countable dimensional $k$ vector space $V$ is said to be algebraic if the map $H \times V \rightarrow V$, defined by $(h, v) \mapsto h . v$, is a morphism.

For an ind-group $H$ and ind-variety $Y$, we say that $Y$ is an $H$-variety if the group $H$ acts on $Y$ such that the action $H \times Y \rightarrow Y$ is a morphism of ind-varieties.

(B.5) Proposition [Sa]. For an ind-group $H$, the Zariski tangent space $T_{e}(H)$ at the identity element $e$ is endowed with a natural Lie algebra structure. We denote this Lie algebra by Lie $H$.

Moreover, if $\alpha: H \rightarrow K$ is a group morphism between two ind-groups, then the induced map $(d \alpha)_{e}:$ Lie $H \rightarrow$ Lie $K$ is a Lie algebra homomorphism.

Proof. Denote $k[H]$ by $A$. The multiplication map $\mu=\mu_{H}: H \times H \rightarrow H$ taking $\left(h_{1}, h_{2}\right) \mapsto h_{1} h_{2}$ induces a continuous homomorphism $\mu^{*}: A \rightarrow k[H \times H]$. There is a canonical inclusion $A \otimes A \hookrightarrow k[H \times H]$, and it is easy to see that the image is dense in $k[H \times H]$. So we denote $k[H \times H]$ by $A \hat{\otimes} A$, and view it as a certain completion of $A \otimes A$. Let $\epsilon: A \rightarrow k$ be the homomorphism, taking $f \mapsto f(e)$. Let $\mathfrak{m}=\operatorname{ker} \epsilon$. Then for any $f \in \mathfrak{m}$

$$
\mu^{*} f-f \otimes 1-1 \otimes f \in \mathfrak{m} \hat{\otimes} \mathfrak{m},
$$

where $\mathfrak{m} \hat{\otimes} \mathfrak{m}$ denotes the closure of $\mathfrak{m} \otimes \mathfrak{m}$ in $A \hat{\otimes} A$.

A continuous derivation $D: A \rightarrow A$ is said to be invariant if $L_{h}^{*} \circ D=D \circ L_{h}^{*}$, for all $h \in H$, where $L_{h}^{*}: A \rightarrow A$ is the algebra homomorphism induced from the left translation map $L_{h}: H \rightarrow H$ taking $g \mapsto h g$. The set Der $A$ of continuous invariant derivations of $A$ is a Lie algebra under

$$
\left[D_{1}, D_{2}\right]:=D_{1} \circ D_{2}-D_{2} \circ D_{1}, \quad D_{1}, D_{2} \in \operatorname{Der} A \text {. }
$$

Define the map $\eta: T_{e}(H) \rightarrow \operatorname{Der} A$ as follows. Take $v \in T_{e}(H)$. Then $v \in$ $T_{e}\left(H_{n}\right)$ for some $n$ (where $H_{n}$ is the filtration of $H$ ). By definition, $T_{e}\left(H_{n}\right)=$ $\operatorname{Hom}_{k}\left(\mathfrak{m}_{n} / \mathfrak{m}_{n}^{2}, k\right)$, where $\mathfrak{m}_{n}:=\left\{f \in k\left[H_{n}\right]: f(e)=0\right\}$ is the maximal ideal of $k\left[H_{n}\right]$ corresponding to the point $e$. In particular, $v$ gives rise to a $k$-linear map $\hat{v}: \mathfrak{m}_{n} \rightarrow k$. Let $\bar{v}: A \rightarrow k$ be the continuous linear map such that $\bar{v}(1)=0$, and $\bar{v}_{\left.\right|_{\mathfrak{m}}}=\hat{v} \circ \pi_{n}$, where $\pi_{n}: \mathfrak{m} \rightarrow \mathfrak{m}_{n}$ is the canonical restriction map. Now the map $\eta(v): A \rightarrow A$ is defined by

$$
\eta(v)=(I \hat{\otimes} \bar{v}) \circ \mu^{*},
$$

where $I: A \rightarrow A$ is the identity map and $I \hat{\otimes} \bar{v}: A \hat{\otimes} A \rightarrow A \hat{\otimes} k=A$ is the completion of the map $I \otimes \bar{v}$. By using (1), we get that $\eta(v)$ is a derivation. Further, it can be seen that $\eta(v)$ is invariant and hence $\eta(v) \in \operatorname{Der} A$.

Conversely, we define a map $\xi:$ Der $A \rightarrow T_{e}(H)$ as follows. Take $D \in \operatorname{Der} A$ and consider $\epsilon \circ D_{\left.\right|_{\mathfrak{m}}}: \mathfrak{m} \rightarrow k$. Since $D$ is continuous, there exists some $n$ such that $\epsilon \circ D_{\left.\right|_{\mathfrak{m}}}$ factors through $\mathfrak{m}_{n}$, giving rise to a map (denoted ) $\beta_{D}: \mathfrak{m}_{n} \rightarrow k$. Since $D$ is a derivation, $\beta_{D}\left(\mathfrak{m}_{n}^{2}\right)=0$ and hence $\beta_{D}$ gives rise to an element $\hat{\beta}_{D} \in T_{e}\left(H_{n}\right)$. Now set $\xi(D)=\hat{\beta}_{D}$.

It can be easily seen that $\xi \circ \eta$ and $\eta \circ \xi$ both are the identity maps, in particular, $\eta$ and $\xi$ are isomorphisms. We now transport the Lie algebra structure from Der $A$ to $T_{e}(H)$ (via $\eta$ ).

Finally, we prove that for any group morphism $\alpha: H \rightarrow K$, the induced map 
To prove this, it suffices to show that the following diagram is commutative (for any $v \in$ Lie $H$ ):

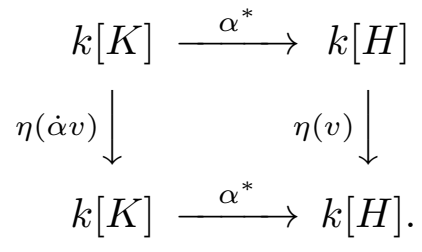

Take $f \in \mathfrak{m}_{K}$, where $\mathfrak{m}_{K} \subset k[K]$ is the maximal ideal corresponding to the point $e$. Then, by the definition of the map $\eta$,

$$
\begin{aligned}
\eta(v)\left(\alpha^{*} f\right) & =(I \hat{\otimes} \bar{v}) \mu_{H}^{*}\left(\alpha^{*} f\right), \quad \text { and } \\
\eta(\dot{\alpha} v) f & =(I \hat{\otimes}(\overline{\dot{\alpha} v})) \mu_{K}^{*}(f) .
\end{aligned}
$$

Further,

$$
\begin{aligned}
\alpha^{*} \eta(\dot{\alpha} v) f & =\left(\alpha^{*} \hat{\otimes}(\overline{\dot{\alpha} v})\right) \mu_{K}^{*}(f), \quad \text { whereas } \\
\overline{\dot{\alpha} v} & =\bar{v} \circ \alpha^{*}, \quad \text { and } \\
\left(\alpha^{*} \hat{\otimes} \alpha^{*}\right) \circ \mu_{K}^{*} & =\mu_{H}^{*} \circ \alpha^{*} .
\end{aligned}
$$

Now combining $(2)-(6)$, we get the commutativity of the diagram $(*)$. This proves the proposition.

(B.6) Lemma. An algebraic representation $\theta$ of an ind-group $H$ in a (countable dimensional) vector space $V$ induces (on 'differentiation' as defined below) a representation $d \theta$ of the Lie algebra Lie $(H)$ on the same space $V$.

Proof. Fix $v \in V$ and consider the map $\theta_{v}: H \rightarrow V$, taking $h \mapsto h v$. Consider the derivative $\left(d \theta_{v}\right)_{e}: T_{e}(H)=\operatorname{Lie}(H) \rightarrow T_{v}(V) \approx V$. Then the representation $d \theta$ : Lie $(H) \times V \rightarrow V$ is defined as $(x, v) \mapsto\left(d \theta_{v}\right)_{e}(x)$. We claim that $d \theta$ is a Lie algebra representation:

We abbreviate $d \theta(x, v)$ by $x . v$. For any $v \in V$, define the evaluation map $e(v)$ : $k[V] \rightarrow k$ by $e(v) f=f(v)$, for $f \in k[V]$. Fix any $v_{0} \in V$. Then $v \in T_{v_{0}}(V) \approx V$ can be thought of (by the definition of the tangent vector) as a $k$-linear map $\bar{v}$ : $k[V] \rightarrow k$, such that $\bar{v}(1)=0$. If $v, w \in T_{v_{0}}(V)$ are such that $\bar{v}_{V_{V^{*}}}=\bar{w}_{V_{V^{*}}}$, then $\bar{v}=\bar{w}$, where $V^{*} \subset k[V]$ denotes the full vector space dual of $V$. Moreover, as is easy to see,

$$
\bar{v}_{V_{V^{*}}}=e(v)_{\left.\right|_{V^{*}}} .
$$

By definition (for any $v \in V$ and $x \in T_{e}(H)$ )

$$
\overline{x \cdot v}=(\bar{x} \hat{\otimes} e(v)) \circ \theta^{*},
$$

where $\theta: H \times V \rightarrow V$ is the representation. Since $\theta$ is linear in the $V$-variable, 
Consider the following commutative diagram, where $A=k[H]$, and $I$ stands for the identity maps.

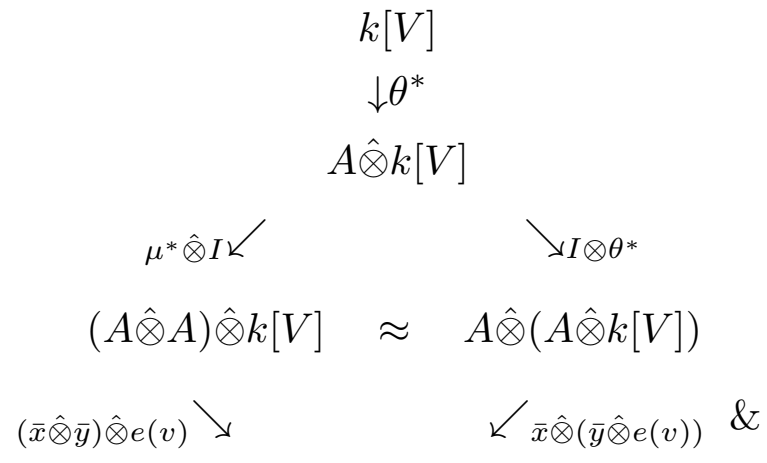

$k \otimes k \otimes k=k$.

The commutativity of the above diagram and (1)-(3) give the following (for any $x, y \in T_{e}(H)$ and $\left.v \in V\right)$.

$$
e(x \cdot(y \cdot v))_{\left.\right|_{V^{*}}}=\left(((\bar{x} \hat{\otimes} \bar{y}) \hat{\otimes} e(v)) \circ\left(\mu^{*} \hat{\otimes} I\right) \circ \theta^{*}\right)_{V_{V^{*}}} .
$$

By (4) we get

$$
(e(x \cdot(y \cdot v))-e(y \cdot(x \cdot v)))_{V_{V^{*}}}=\left(((\bar{x} \hat{\otimes} \bar{y}-\bar{y} \hat{\otimes} \bar{x}) \hat{\otimes} e(v)) \circ\left(\mu^{*} \hat{\otimes} I\right) \circ \theta^{*}\right)_{V^{*}} \cdot
$$

But, as can be easily seen from the definition of the bracket in $T_{e}(H)$ (cf. proof of Proposition B.5),

$$
(\bar{x} \hat{\otimes} \bar{y}-\bar{y} \hat{\otimes} \bar{x}) \circ \mu^{*}=\overline{[x, y]} .
$$

In particular,

$$
\begin{aligned}
e(x \cdot(y \cdot v)-y \cdot(x \cdot v))_{\left.\right|_{V^{*}}} & =\left((\overline{[x, y]} \hat{\otimes} e(v)) \circ \theta^{*}\right)_{\left.\right|_{V^{*}}}, \\
& =(\overline{[x, y] \cdot v})_{\left.\right|_{V^{*}}}, \text { by }(2) \\
& =e([x, y] \cdot v)_{\left.\right|_{V^{*}}} \text {, by }(1) .
\end{aligned}
$$

This gives that $x \cdot(y \cdot v)-y \cdot(x \cdot v)=[x, y] \cdot v$, proving the lemma.

(B.7) Definitions. For any ind-variety $Y$, by an algebraic vector bundle of rank $r$ over $Y$, we mean an ind-variety $E$ together with a morphism $\theta: E \rightarrow Y$ such that (for any $n$ ) $E_{n} \rightarrow Y_{n}$ is an algebraic vector bundle of rank $r$ over the (finite dimensional) variety $Y_{n}$, where $\left\{Y_{n}\right\}$ is the filtration of $Y$ giving the ind-variety structure and $E_{n}:=\theta^{-1}\left(Y_{n}\right)$. If $r=1$, we call $E$ an algebraic line bundle over $Y$.

Let $E$ and $F$ be two algebraic vector bundles over $Y$. Then a morphism (of ind-varieties) $\varphi: E \rightarrow F$ is called a bundle morphism if the following diagram is commutative :

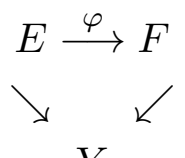


and moreover $\varphi_{\mid E_{n}}: E_{n} \rightarrow F_{n}$ is a bundle morphism for all $n$. In particular, we have the notion of isomorphism of vector bundles over $Y$.

We define Pic $Y$ as the set of isomorphism classes of algebraic line bundles on $Y$. It is clearly an abelian group under the tensor product of line bundles.

We similarly define the notion of principal $G$-bundles on an ind-variety (for a finite dimensional algebraic group $G$ ).

For an ind-group $H$ and $H$-variety $Y$ (cf. §B.4), an algebraic vector bundle $\theta: E \rightarrow Y$ is said to be an $H$-equivariant vector bundle if the ind-variety $E$ is also an $H$-variety such that the following diagram is commutative:

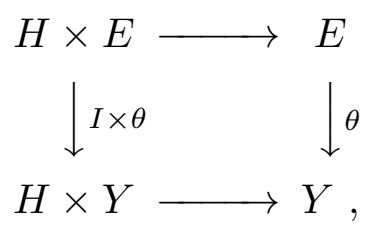

and moreover for any $y \in Y$ and $h \in H$ the fiber map $h \times \theta^{-1} y \rightarrow \theta^{-1}(h y)$ is linear.

\section{Appendix C. Affine Kac-Moody groups and their flag varieties.}

Let $G$ be a connected simply-connected simple algebraic group and let $\mathcal{G}:=$ $G(\mathbb{C}((t))), \mathcal{P}:=G(\mathbb{C}[[t]])$. We fix a Borel subgroup $B \subset G$ and a maximal torus $T \subset B$, and define the standard Borel subgroup $\mathcal{B}$ of $\mathcal{G}$ as $e v_{0}^{-1}(B)$, where $e v_{0}: \mathcal{P}=$ $G(\mathbb{C}[[t]]) \rightarrow G$ is the group homomorphism induced from the $\mathbb{C}$-algebra homomorphism $\mathbb{C}[[t]] \rightarrow \mathbb{C}$, taking $t \mapsto 0$.

Let $N(T)$ be the normalizer of $T$ in $G$ and consider the set Mor $\left(\mathbb{C}^{*}, N(T)\right)$ of all the regular maps $f: \mathbb{C}^{*} \rightarrow N(T)$, which is a group under poinwise multiplication. Then $T$ can be thought of as a (normal) subgroup of Mor $\left(\mathbb{C}^{*}, N(T)\right)$ consisting of constant loops in $T$. Then the affine Weyl group $\widetilde{W}$ of $G$ is by definition $\widetilde{W}=\operatorname{Mor}\left(\mathbb{C}^{*}, N(T)\right) / T$. Clearly the (finite) Weyl group $W:=N(T) / T$ of $G$ is a subgroup of $\widetilde{W}$.

(C.1) Bruhat Decomposition. We can view $\operatorname{Mor}\left(\mathbb{C}^{*}, N(T)\right)$ as a subgroup of $\mathcal{G}$. In particular, any element $w \in \operatorname{Mor}\left(\mathbb{C}^{*}, N(T)\right)$ can be thought of as an element (denoted by the same symbol) $w$ of $\mathcal{G}$. The generalised flag variety $X:=\mathcal{G} / \mathcal{P}$ has the following Bruhat decomposition:

$$
X=\bigcup_{\mathfrak{w} \in \widetilde{W} / W} \mathcal{B} \mathfrak{w} \mathcal{P} / \mathcal{P},
$$

where the notation $\mathcal{B} \mathfrak{w} \mathcal{P} / \mathcal{P}$ means $\mathcal{B} w \mathcal{P} / \mathcal{P}$ for any choice of the coset representative $w$ of $\mathfrak{w}$. (The set $\mathcal{B} \mathfrak{w} \mathcal{P} / \mathcal{P}$ is independent of these choices.) Moreover the union in (1) is disjoint.

The affine Weyl group $\widetilde{W}$ is a Coxeter group and hence has a Bruhat partial order $\leq$. This induces a partial order (again denoted by) $\leq$ in $\widetilde{W} / W$ defined by

$$
\mathfrak{u}:=u \bmod W \leq \mathfrak{v} \quad(\text { for } u, v \in \widetilde{W})
$$

if and only if there exists a $w \in W$ such that 
We define the generalised Schubert variety $X_{\mathfrak{w}}$ (for any $\mathfrak{w} \in \widetilde{W} / W$ ) by

$$
X_{\mathfrak{w}}:=\bigcup_{\mathfrak{v} \leq \mathfrak{w}} \mathcal{B} \mathfrak{v} \mathcal{P} / \mathcal{P}
$$

Then clearly $X_{\mathfrak{v}} \subseteq X_{\mathfrak{w}}$ if and only if $\mathfrak{v} \leq \mathfrak{w}$.

(C.2) Definition. Let $\mathfrak{g}$ be the Lie algebra of $G$. We define the adjoint representation Ad of $\mathcal{G}$ in $\widetilde{\mathfrak{g}}_{\text {comp }}$ as follows (cf. [PS, Proposition 4.3.2]):

Embed $G \hookrightarrow S L_{N}$ and define for any $g \in \mathcal{G}, Y \in \mathfrak{g} \otimes \mathbb{C}((t))$ and $z \in \mathbb{C}$

$$
\operatorname{Ad}(g)(Y+z K)=g Y g^{-1}+\left(z-\operatorname{Res}_{t=0}\left\langle g^{-1} \frac{d g}{d t}, Y\right\rangle_{t}\right) K,
$$

where $\langle,\rangle_{t}$ is the $\mathbb{C}((t))$-bilinear extension of the normalized Killing form $\langle$,$\rangle on \mathfrak{g}$ (cf. $§$ A.1).

The following lemma is well known, but we give a proof for completeness. Even though we do not need, a more general lemma (where the base field $\mathbb{C}$ is replaced by any $\mathbb{C}$-algebra) for $G=S L_{N}$ is proved by Faltings (cf. [BL, Appendix A]).

(C.3) Lemma. Let $\pi: \tilde{\mathfrak{g}} \rightarrow$ End $W$ be an integrable highest weight (in particular irreducible) representation of $\tilde{\mathfrak{g}}$. Then there exists a unique group homomorphism $\hat{\pi}: \mathcal{G} \rightarrow P G L(W)$, such that the following holds for any $g \in \mathcal{G}$ and $X \in \tilde{\mathfrak{g}}_{\text {comp }}$ :

$$
\hat{\pi}(g) \bar{\pi}(X) \hat{\pi}(g)^{-1}=\bar{\pi}(\operatorname{Ad} g X),
$$

where $\bar{\pi}: \tilde{\mathfrak{g}}_{\mathrm{comp}} \rightarrow$ End $W$ is the extension of $\pi$ (cf. $\S \mathrm{A} .2$ ), $P G L(W):=G L(W) / \mathbb{C}^{*}$, and $G L(W)$ is the group of all linear automorphisms of $W$. (We view $\hat{\pi}(g) \bar{\pi}(X) \hat{\pi}(g)^{-1}$ as an element of End $W$ by taking any lift of $\hat{\pi}(g)$ in $G L(W)$.)

Proof. Fix $g \in \mathcal{G}$. We first prove that if there exists an element $\theta \in P G L(W)$ such that $\theta \bar{\pi}(X) \theta^{-1}=\bar{\pi}(\operatorname{Ad} g X)$, for all $X \in \tilde{\mathfrak{g}}_{\text {comp }}$, then $\theta$ is unique:

For, if possible, let $\theta_{1}$ be another such element. Then

$$
\left(\theta_{1}^{-1} \theta\right) \bar{\pi}(X)\left(\theta_{1}^{-1} \theta\right)^{-1}=\bar{\pi}(X), \quad \text { for all } X \in \tilde{\mathfrak{g}}_{\mathrm{comp}} .
$$

But $W$ being irreducible, $\left(\theta_{1}^{-1} \theta\right)=1$ (in $P G L(W)$ ). This proves the uniqueness assertion.

Define the set

$$
S=\left\{g \in \mathcal{G}: \hat{\pi}(g) \text { is defined satisfying }(*) \text { for all } X \in \tilde{\mathfrak{g}}_{\text {comp }}\right\} .
$$

By uniqueness, it is clear that $S$ is a subgroup of $\mathcal{G}$ and moreover the map $\hat{\pi}: S \rightarrow$ $P G L(W)$ is a group homomorphism. We next prove that $S=\mathcal{G}$ :

For any root vector $X \in \mathfrak{g}$ and $p \in \mathbb{C}((t))$, define

$$
\hat{\pi}(\exp (X \otimes p))=\exp (\bar{\pi}(X \otimes p)) .
$$

(Since $X$ is a root vector and $W$ is integrable, $\bar{\pi}(X \otimes p) \in$ End $W$ is locally nilpotent, in particular, $\exp (\bar{\pi}(X \otimes p))$ is well defined.) It is easy to see that $\hat{\pi}(\exp (X \otimes p))$, as defined by $(1)$, satisfies $(*)$ for every $X \in \tilde{\mathfrak{g}}_{\text {comp }}$. Further, by a result of Steinberg, the group generated by the elements $\exp (\bar{\pi}(X \otimes p))$ is the whole group $\mathcal{G}$. This proves the lemma. 
(C.4) Central Extension. Recall the definition of the integrable highest weight (irreducible) $\tilde{\mathfrak{g}}$-module $W_{0}=L(\mathbb{C}, 1)$ with central charge 1 from Lemma (A.5), where $\mathbb{C}$ is the trivial one-dimensional representation of $\mathfrak{g}$. By Lemma (C.3), there exists a group homomorphism $\hat{\pi}: \mathcal{G} \rightarrow P G L\left(W_{0}\right)$. We define the group $\tilde{\mathcal{G}}$ as the pull-back $\hat{\pi}^{*}\left(G L\left(W_{0}\right)\right)$ :

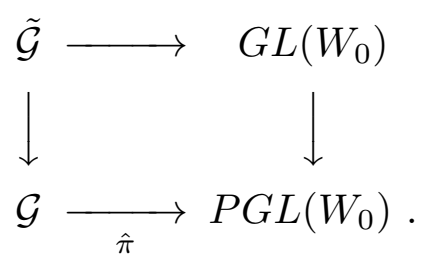

Then $\tilde{\mathcal{G}}$ is a central extension:

$$
1 \rightarrow \mathbb{C}^{*} \rightarrow \tilde{\mathcal{G}} \stackrel{\pi}{\longrightarrow} \mathcal{G} \rightarrow 1 .
$$

By the very definition of $\tilde{\mathcal{G}}$, the $\tilde{\mathfrak{g}}$-module $W_{0}$ becomes a $\tilde{\mathcal{G}}$-module. In particular, the tensor product $W_{0}^{\otimes m}$ (for $m \geq 0$ ) acquires a canonical $\tilde{\mathcal{G}}$-module structure. Since the integrable $\tilde{\mathfrak{g}}$-module $L(\mathbb{C}, m)$ with central charge $m \geq 0$ is a $\tilde{\mathfrak{g}}$-submodule of $W_{0}^{\otimes m}$, it is easy to see (using (1) of the proof of Lemma C.3) that $\tilde{\mathcal{G}}$ keeps $L(\mathbb{C}, m) \subset W_{0}^{\otimes m}$ stable. In particular, $L(\mathbb{C}, m)$ acquires a canonical $\tilde{\mathcal{G}}$-module structure.

(C.5) Realizing $X$ as an ind-variety via representation theory. Define the filtration $\left\{X_{n}\right\}_{n \geq 0}$ of $X$ as follows:

$$
X_{n}:=\cup X_{\mathfrak{w}},
$$

where the union is taken over those $\mathfrak{w}=w \bmod W \in \widetilde{W} / W$ such that $\ell(w) \leq n$.

Fix any integer $\ell>0$ and consider the irreducible $\tilde{\mathfrak{g}}-$ module $L(\mathbb{C}, \ell)$. From the Bruhat decomposition, it is easy to see that the map $i=i_{\ell}: X \rightarrow \mathbb{P}(L(\mathbb{C}, \ell)), g \mathcal{P} \mapsto$ $\left[g v_{o}\right]$ (where $v_{o}$ is the highest weight vector of $L(\mathbb{C}, \ell)$ and $\mathbb{P}(L(\mathbb{C}, \ell)$ ) denotes the space of lines in $L(\mathbb{C}, \ell)$ ) is injective. As proved in [Sl, §2.4], for any $n$, there exists a finite dimensional subspace $W_{n} \subset L(\mathbb{C}, \ell)$ such that $i\left(X_{n}\right) \subset \mathbb{P}\left(W_{n}\right)$ and moreover $i\left(X_{n}\right)$ is Zariski closed in $\mathbb{P}\left(W_{n}\right)$. We endow $X_{n}$ with the projective (reduced) variety structure so that $i_{\mid X_{n}}$ is a closed immersion. This makes $X$ into a projective ind-variety. Further, the ind-variety structure does not depend upon the particular choice of $\ell>0$ (cf. [Sl, §2.5]). Equipped with this ind-variety structure, we denote $X$ by $X_{\text {rep }}$.

For any $\mathfrak{w} \in \widetilde{W} / W$ the generalised Schubert variety $X_{\mathfrak{w}}$ is an irreducible Zariski closed subset of $X_{\text {rep }}$. We endow $X_{\mathfrak{w}}$ with the projective (reduced) variety structure so that $X_{\mathfrak{w}} \hookrightarrow X$ is a closed immersion. Then $\mathcal{B} \mathfrak{w} \mathcal{P} / \mathcal{P} \subset X_{\mathfrak{w}}$ is an open subset which is biregular isomorphic with the affine space $\mathbb{C}^{\ell(\mathfrak{w})}$, where $\ell(\mathfrak{w})$ is the length of the smallest element in the coset $\mathfrak{w}$.

Since $X_{n}$ is a variety, we can equip $X_{n}$ with the (Hausdorff) analytic topology and put the inductive limit topology on $X$. The decomposition (1) of $\S($ C.1) provides a cellular decomosition of $X$, making it into a CW complex.

Following [Sl,§2.7], we define the homogeneous line bundles on $X_{\text {rep }}$ :

(C.6) Definition. For any countable dimensional vector space $V$, we first define the tautological line bundle $\mathcal{L}_{V}$ on $\mathbb{P}(V)$ as follows: Consider the subset 
Then $\mathcal{L}_{V}$ is a Zariski-closed subset of the ind-variety $\mathbb{P}(V) \times V$. We equip $\mathcal{L}_{V}$ with the ind-variety structure so that $\mathcal{L}_{V} \hookrightarrow \mathbb{P}(V) \times V$ is a closed immersion. Now the projection on the first factor $\mathcal{L}_{V} \rightarrow \mathbb{P}(V)$ realizes $\mathcal{L}_{V}$ as an algebraic line bundle on $\mathbb{P}(V)$.

For any $\ell>0$, define the algebraic line bundle $\mathfrak{L}\left(\ell \chi_{0}\right)$ on $X$ as the pull-back of the dual $\mathcal{L}^{*}$ of the tautological line bundle $\mathcal{L}=\mathcal{L}_{L(\mathbb{C}, \ell)}$ on $\mathbb{P}(L(\mathbb{C}, \ell))$ via the embedding $i_{\ell}: X \rightarrow \mathbb{P}(L(\mathbb{C}, \ell))$ of the above section. For any integer $\ell<0$, we define the line bundle $\mathfrak{L}\left(\ell \chi_{0}\right)$ as the dual $\mathfrak{L}\left(-\ell \chi_{0}\right)^{*}$ and for $\ell=0, \mathfrak{L}\left(\ell \chi_{0}\right)$ is defined to be the trivial line bundle. It is easy to see (cf. [Sl, §2.7]) that the line bundle $\mathfrak{L}\left(\ell \chi_{0}\right)$ is isomorphic with the line bundle $\mathfrak{L}\left(\chi_{0}\right)^{\otimes \ell}$.

The group $\mathcal{G}$ acts (set theoretically) on $X=\mathcal{G} / \mathcal{P}$ via $g(h \mathcal{P})=g h \mathcal{P}$, for $g, h \in \mathcal{G}$. We denote the action of $g \in \mathcal{G}$ on $X$ by $L_{g}$. This action lifts to an action of the group $\tilde{\mathcal{G}}$ on the line bundle $\mathfrak{L}\left(\ell \chi_{0}\right)($ for $\ell<0)$ via

$$
g(x, v)=\left(L_{\pi(g)} x, g v\right), \quad \text { for any } g \in \tilde{\mathcal{G}}, x \in X \text { and } v \in i_{\ell}(x)
$$

where $\pi: \tilde{\mathcal{G}} \rightarrow \mathcal{G}$ is the canonical map. Observe that for any fixed $g \in \tilde{\mathcal{G}}$, the action of $g$ on $\mathfrak{L}\left(\ell \chi_{0}\right)$ is an algebraic automorphism of the algebraic line bundle $\mathcal{L}\left(\ell \chi_{0}\right)$ inducing the automorphism $L_{\pi(g)}$ on the base $X$.

Set $\mathfrak{L}_{o}:=\mathfrak{L}\left(-\chi_{0}\right)$, and define the Mumford group (cf. [PS, Remark (i), page 115]) $\operatorname{Aut}\left(\mathfrak{L}_{o}\right)=\{(g, \varphi): g \in \mathcal{G}$ and $\varphi$ is an algebraic automorphism of the line bundle $\mathfrak{L}_{o}$ inducing the map $L_{g}$ on the base $\}$. Then $\operatorname{Aut}\left(\mathfrak{L}_{o}\right)$ is a group under

$$
\left(g_{1}, \varphi_{1}\right)\left(g_{2}, \varphi_{2}\right)=\left(g_{1} g_{2}, \varphi_{1} \varphi_{2}\right)
$$

The projection on the first factor gives a group homomorphism $\delta: \operatorname{Aut}\left(\mathfrak{L}_{o}\right) \rightarrow \mathcal{G}$. Since $\tilde{\mathcal{G}}$ acts on $\mathfrak{L}_{o}$, there is a canonical group homomorphism $\xi: \tilde{\mathcal{G}} \rightarrow \operatorname{Aut}\left(\mathfrak{L}_{o}\right)$ making the following diagram commutative:

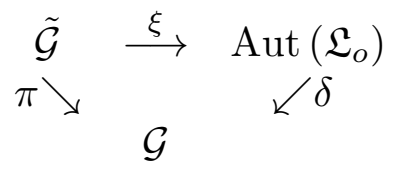

Since $\pi$ is surjective, so is $\delta$. In particular, $\xi$ is an isomorphism.

We also need another 'lattice' description of the ind-variety structure on $X$ (cf. $[\mathrm{KL}, \S 5])$.

(C.7) Realizing $X$ as an ind-variety via lattices. We first consider the case of $G=S L_{N}$. Denote $V=\mathbb{C}^{N}$, and $A=\mathbb{C}[[t]]$. For any $n \geq 0$, consider the set $\mathcal{F}_{n}$ of A-submodules $L \subset V \otimes_{\mathbb{C}} \mathbb{C}((t))$ such that (denoting $V \otimes_{\mathbb{C}} A$ by $L_{o}$ )

$$
t^{n} L_{o} \subset L \subset t^{-n} L_{o} \text {, and } \operatorname{dim} L / t^{n} L_{o}=n N
$$

Let $V_{n}:=t^{-n} L_{o} / t^{n} L_{o}$ be the complex vector space of dimension $2 n N$. Then the multiplication by $t$ induces a nilpotent endomorphism $\bar{t}_{n}$ of $V_{n}$ and hence $1+\bar{t}_{n}$ is a (unipotent) automorphism of $V_{n}$. In particular, $1+\bar{t}_{n}$ induces a biregular isomorphism of the Grassmannian $\operatorname{Gr}(n N, 2 n N)$ of $n N$-dimensional subspaces of the $2 n N$-dimensional space $V_{n}$. Let $\operatorname{Gr}(n N, 2 n N)^{1+\bar{t}_{n}}$ denote its fixed point. Then 
We pull the (reduced) subvariety structure of $\operatorname{Gr}(n N, 2 n N)^{1+\bar{t}_{n}}$ via $j_{n}$ to equip $\mathcal{F}_{n}$ with a projective variety structure. We next claim that the canonical inclusion $\mathcal{F}_{n} \hookrightarrow \mathcal{F}_{n+1}$ is a closed immersion:

Consider the commutative diagram:

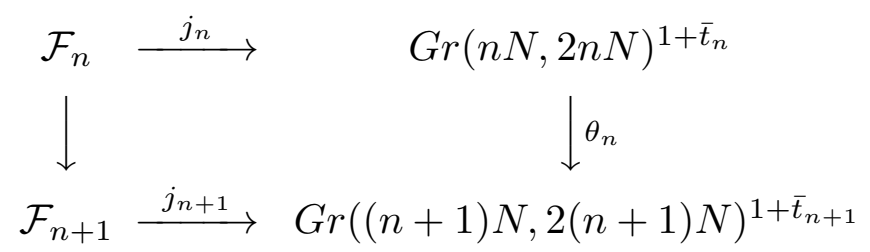

where the map $\theta_{n}$ takes $W \subset t^{-n} L_{o} / t^{n} L_{o} \approx t^{n-1} V \oplus t^{n-2} V \oplus \cdots \oplus t^{-n} V \mapsto$ $t^{n} V \oplus W$. It is easy to see that $\theta_{n}$ is a closed immersion. This equips $\mathcal{F}=\cup_{n>0} \mathcal{F}_{n}$ with a projective ind-variety structure.

Let $\mathcal{G}^{o}:=S L_{N}(\mathbb{C}((t)))$ and $\mathcal{P}^{o}:=S L_{N}(A)$ and set $X^{o}=\mathcal{G}^{o} / \mathcal{P}^{o}$. By virtue of the following lemma, the map $\beta: X^{o} \rightarrow \mathcal{F}$ is a bijection. By transporting the projective ind-variety structure from $\mathcal{F}$ to $X^{o}$ (via $\beta$ ) we equip $X^{o}$ with a projective ind-variety structure. With this structure we denote $X^{o}$ by $X_{\text {lat }}^{o}$. We also define the filtration $\hat{X}_{n}^{o}$ of $X^{o}$ by

$$
\hat{X}_{n}^{o}=\beta^{-1}\left(\mathcal{F}_{n}\right) .
$$

The group $\mathcal{G}^{o}$ acts canonically on $V \otimes \mathbb{C}((t))$.

(C.8) Lemma. The map $g \mathcal{P}^{o} \mapsto g L_{o}\left(\right.$ for $\left.g \in \mathcal{G}^{o}\right)$ induces a bijection $\beta: X^{o} \rightarrow \mathcal{F}$.

Proof. Fix $g \in \mathcal{G}^{\circ}$. It is easy to see that there exists some $n$ (depending upon $g$ ) such that

$$
t^{n} L_{o} \subset g L_{o} \subset t^{-n} L_{o}
$$

Of course $g L_{o}$ is $t$-stable. We next calculate the dimension of $g L_{o} / t^{n} L_{o}$ :

By the Bruhat decomposition (1) of $\S($ C.1), it suffices to assume that $g$ is a morphism $\mathbb{C}^{*} \rightarrow D$ taking $1 \mapsto 1$, where $D$ is the diagonal subgroup of $S L_{N}$. Write

$$
g(t)=\left(\begin{array}{ccc}
t^{n_{1}} & & 0 \\
& \ddots & \\
0 & & t^{n_{N}}
\end{array}\right), \quad \text { for } t \in \mathbb{C}^{*} \text { and } n_{i} \in \mathbb{Z}
$$

Then $\sum n_{i}=0$. Now

$$
\operatorname{dim}\left(g L_{o} / t^{n} L_{o}\right)=\left(n-n_{1}\right)+\cdots+\left(n-n_{N}\right)=N n-\sum n_{i}=N n .
$$

This proves that $g L_{o} \in \mathcal{F}_{n}$.

Conversely, take $L \in \mathcal{F}_{n}$. Since $A$ is a PID and $t^{k} L_{o}$ is $A$-free of rank $N$ (for any $k \in \mathbb{Z})$, we get that $L$ is $A$-free of rank $N$. Further, $L \otimes_{A} \mathbb{C}((t)) \rightarrow V((t))$ is an isomorphism, where $V((t)):=V \otimes_{\mathbb{C}} \mathbb{C}((t))$. Let $\left\{e_{1}, \ldots, e_{N}\right\}$ be the standard $\mathbb{C}$-basis of $V$ and take an $A$-basis $\left\{v_{1}, \ldots, v_{N}\right\}$ of $L$. Now define the $\mathbb{C}((t))$-linear automorphism $g$ of $V((t))$ by $g e_{i}=v_{i}(1 \leq i \leq N)$. We prove that $\operatorname{det} g$ is a unit of $A$ : Write $\operatorname{det} g=t^{k} u$, where $k \in \mathbb{Z}$ and $u$ is a unit of $A$. Consider the $\mathbb{C}((t))$-linear automorphism $\alpha$ of $V((t))$ defined by

$$
\alpha e_{i}=e_{i}, \quad \text { for } 1 \leq i<N,
$$


Then $\operatorname{det}(g \alpha)=1$, and $t^{n+|k|} L_{o} \subset(g \alpha) L_{o} \subset t^{-n-|k|} L_{o}$. Hence, by the first part of the proof, we get

$$
\operatorname{dim}\left(\frac{g \alpha\left(L_{o}\right)}{t^{n+|k|} L_{o}}\right)=(n+|k|) N .
$$

On the other hand,

$$
\begin{aligned}
\operatorname{dim}\left(\frac{g \alpha\left(L_{o}\right)}{t^{n+|k|} L_{o}}\right) & =\operatorname{dim} \frac{g L_{o}}{t^{n} L_{o}}+|k| N+k \\
& =N n+|k| N+k \quad\left(\text { since } L \in \mathcal{F}_{n}\right) .
\end{aligned}
$$

Now combining (2) and (3), we get $k=0$, hence $(g \alpha) L_{o}=g L_{o}=L$. This proves the surjectivity of $\beta$. The injectivity of $\beta$ is clear. This proves the lemma.

(C.9). We now come to the case of general (connected, simply-connected, simple) $G$. Fix an embedding $G \hookrightarrow S L_{N}$. This gives rise to an embedding

$$
X=\mathcal{G} / \mathcal{P} \hookrightarrow X^{o}=\mathcal{G}^{o} / \mathcal{P}^{o} .
$$

The filtration $\hat{X}_{n}^{o}$ of $X^{o}$ (given in $\S$ C.7) on restriction gives the filtration $\hat{X}_{n}$ of $X$, i.e.,

$$
\hat{X}_{n}=\hat{X}_{n}^{o} \cap X
$$

In (a subsequent) Lemma (C.11), we prove that $\hat{X}_{n}$ is a Zariski closed subset of $\hat{X}_{n}^{o}$. This allows us to put the reduced subvariety structure on $\hat{X}_{n}$ making $X$ into a projective ind-variety. Equipped with this ind-variety structure, we denote $X$ by $X_{\text {lat. }}$

(C.10) Lemma. The two filtrations $X_{n}$ and $\hat{X}_{n}$ of $X$ are compatible, i.e., for every $n$ there exists $k(n)$ such that

$$
X_{n} \subseteq \hat{X}_{k(n)} \text { and } \hat{X}_{n} \subseteq X_{k(n)}
$$

Proof. Fix a maximal torus $T \subset G$ and an embedding $G \hookrightarrow S L_{N}$ such that $T$ goes inside the diagonal subgroup $D$ of $S L_{N}$. Now there is a bijection $\widetilde{W} W \simeq$ $\operatorname{Mor}_{1}\left(\mathbb{C}^{*}, T\right)$, where Mor $_{1}$ denotes the set of morphisms $\mathbb{C}^{*} \rightarrow T$ such that $1 \mapsto 1$. Since the set $\{\mathfrak{w} \in \widetilde{W} / W: \ell(w) \leq n\}$ is finite, it is easy to see that $X_{n} \subset \hat{X}_{k(n)}$ (for some large enough $k(n))$.

Conversely (for a fixed $n$ ), we want to show for all but finitely many $\mathfrak{w} \in \widetilde{W} / W$, $(\mathcal{B} \mathfrak{w} \mathcal{P} / \mathcal{P}) \cap \hat{X}_{n}=\phi:$ Represent $\mathfrak{w}$ as a morphism $\mathbb{C}^{*} \rightarrow T \hookrightarrow D$

$$
z \mapsto\left(\begin{array}{ccc}
z^{n_{1}(\mathfrak{w})} & & \\
& \ddots & \\
& & z^{n_{N}(\mathfrak{w})}
\end{array}\right) .
$$

We first claim that any $\mathfrak{w}$ such that $n_{i}(\mathfrak{w})<-n$ (for some $i$ ) satisfies $(\mathcal{B} \mathfrak{w} \mathcal{P} / \mathcal{P}) \cap$ $\hat{X}_{n}=\phi:$ If for some $b \in \mathcal{B}, b \mathfrak{w} L_{o} \in \mathcal{F}_{n}$, then clearly $\mathfrak{w} L_{o} \in b^{-1} \mathcal{F}_{n}=\mathcal{F}_{n}$, a contradiction to the choice of $\mathfrak{w}$. Now observe that the set $\left\{\mathfrak{w} \in \widetilde{W} / W: n_{i}(\mathfrak{w}) \geq-n\right.$ for all $i\}$ is finite, since $\sum n_{i}(\mathfrak{w})=0$. From this, it follows that $\hat{X}_{n} \subset X_{k(n)}$, for some large enough $k(n)$. This proves the lemma. 
(C.11) Lemma. With the notation as above, $\hat{X}_{n}$ is a Zariski closed subset of $\hat{X}_{n}^{o}$ (for any $n \geq 0$ ).

Proof. Fix $\mathfrak{w} \in \widetilde{W} / W(\widetilde{W}$ is the affine Weyl group corresponding to $G$ ) and take a coset representative $w$ of $\mathfrak{w}$ of minimal length. Choose any reduced decomposition $w=s_{i_{1}} \ldots s_{i_{p}}$ (where $s_{j}$ 's are the simple reflections in $\widetilde{W}$ ), and consider the BottSamelson-Demazure-Hansen variety $Z_{w}$ defined in $[\mathrm{Sl}, \S 2.3]$. Let $\mathcal{P}_{j}$ be the minimal parabolic subgroup of $\mathcal{G}$ corresponding to the simple reflection $s_{j}$. Recall that, set theoretically, $Z_{w}=\mathcal{P}_{i_{1}} \times \cdots \times \mathcal{P}_{i_{p}} / \mathcal{B}^{p}$, where $\mathcal{B}^{p}$ acts on $\mathcal{P}_{i_{1}} \times \mathcal{P}_{i_{2}} \times \cdots \times \mathcal{P}_{i_{p}}$ from the right via

$$
\left(x_{1}, \ldots, x_{p}\right)\left(b_{1}, \ldots, b_{p}\right)=\left(x_{1} b_{1}, b_{1}^{-1} x_{2} b_{2}, \ldots, b_{p-1}^{-1} x_{p} b_{p}\right),
$$

for $x_{j} \in \mathcal{P}_{i_{j}}$ and $b_{j} \in \mathcal{B}$.

Define the map $\theta_{w}: Z_{w} \rightarrow X$ by $\theta_{w}\left(\left(x_{1}, \ldots, x_{p}\right) \bmod \mathcal{B}^{p}\right)=x_{1} \ldots x_{p} \mathcal{P}$. Since $\operatorname{Im}$ $\theta_{w}=X_{\mathfrak{w}}$ (cf. [Sl, §2.4]), by the above lemma, $\operatorname{Im}\left(i \circ \theta_{w}\right) \subset \hat{X}_{m}^{o}$ for some $m$, where $i: X \hookrightarrow X^{o}$ is the inclusion. By an argument similar to the proof of [Sl, Theorem $2.4]$, it can be easily seen that $i \circ \theta_{w}: Z_{w} \rightarrow X_{\text {lat }}^{o}$ is a morphism. In particular, $Z_{w}$ being projective, $i\left(X_{\mathfrak{w}}\right)$ is closed in $\hat{X}_{m}^{o}$. We now prove that $i\left(\hat{X}_{n}\right)$ is closed in $\hat{X}_{n}^{o}$ :

Observe that $\hat{X}_{n}$ is left $\mathcal{B}$-stable. Fix any $\mathfrak{w} \in \widetilde{W} / W$ such that $\mathcal{B} \mathfrak{w} \mathcal{P} / \mathcal{P} \subset \hat{X}_{n}$. Then we claim that $X_{\mathfrak{w}} \subset \hat{X}_{n}$ : There is an open (dense) subset $Y_{w} \subset Z_{w}$ such that $\theta_{w}\left(Y_{w}\right)=\mathcal{B} \mathfrak{w} \mathcal{P} / \mathcal{P}$. Hence, considering the morphism $i \circ \theta_{w}: Z_{w} \rightarrow X^{o}$, we see that $i \circ \theta_{w}\left(Z_{w}\right) \subset \hat{X}_{n}^{o}$ (since $\hat{X}_{n}^{o}$ is projective). In particular, $X_{\mathfrak{w}} \subset \hat{X}_{n}$ and thus $\hat{X}_{n}$ is a finite union (by Lemma C.10) of Schubert varieties $X_{\mathfrak{w}}$. Now since $i\left(X_{\mathfrak{w}}\right)$ is closed in $\hat{X}_{n}^{o}$, so is $i\left(\hat{X}_{n}\right)$. This proves the lemma.

(C.12) Proposition. The identity map $X_{\mathrm{rep}} \rightarrow X_{\mathrm{lat}}$ is an isomorphism of indvarieties.

Proof. Embed $G \hookrightarrow \operatorname{SL}(N)$ as in $\S($ C.9) and follow the same notation as in $\S \S($ C.7) and (C.9). By definition, $X_{\text {lat }} \hookrightarrow X_{\text {lat }}^{o}$ is a closed immersion. Similarly, we claim that $X_{\text {rep }} \hookrightarrow X_{\text {rep }}^{o}$ is a closed immersion :

Take the integrable highest weight module $L=L(\mathbb{C}, \ell)$ for $\widetilde{\mathfrak{g}^{o}}$ (for any integer $\ell>0$, where $\mathfrak{g}^{o}=s l_{N}$ ), and let $W \subseteq L$ be the (integrable highest weight) $\tilde{\mathfrak{g}}$-module spanned by the highest weight vector of $L$. Then we have

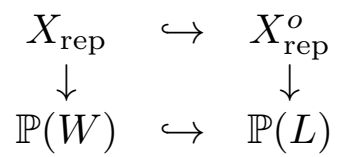

where both the vertical maps are by definition closed immersions, and moreover $\mathbb{P}(W) \hookrightarrow \mathbb{P}(L)$ is of course a closed immersion. This proves that $X_{\text {rep }} \hookrightarrow X_{\text {rep }}^{o}$ is a closed immersion. So, to prove the lemma, we can take $G=S L_{N}$ :

Fix $\mathfrak{w} \in \widetilde{W} / W$ (where $\widetilde{W}$ is the affine Weyl group corresponding to $G=S L_{N}$ ). By the proof of Lemma (C.11) (following the same notation), the map $\theta_{w}: Z_{w} \rightarrow$ $X_{\text {lat }}^{o}$ is a morphism with its image precisely equal to $X_{\mathfrak{w}}^{o}$. We denote $X_{\mathfrak{w}}^{o}$ endowed with the reduced subvariety structure from $X_{\text {rep }}^{o}$ by $X_{\mathfrak{w} \text {,rep }}^{o}$ (and a similar meaning for $X_{\mathfrak{w}, \text { lat }}^{o}$ ). Then the map $\bar{\theta}_{w}: Z_{w} \rightarrow X_{\mathfrak{w} \text {,rep }}^{o}$ (the map $\bar{\theta}_{w}$ at the level of sets is nothing but $\theta_{w}$ ) is a surjective morphism (cf. [Sl, Theorem 2.4]) and moreover $X_{\mathfrak{w}, \text { rep }}^{o}$ is a normal irreducible variety (cf. $\left[\mathrm{Ku}_{1}\right.$, Theorem 2.16]). We claim that the 
First of all, by Lemma (C.10), Image $I_{\mathfrak{w}} \subset \hat{X}_{n}^{o}$, for some $n$. Now the map $\bar{\theta}_{w}: Z_{w} \rightarrow X_{\mathfrak{w}, \text {,rep }}^{o}$ being a proper surjective morphism, the (Zariski) topology on $X_{\mathfrak{w}, \text { rep }}^{o}$ is the quotient topology. Let $\mathcal{U} \subset \hat{X}_{n}^{o}$ be an open subset. Then $\theta_{w}^{-1}(\mathcal{U})=$ $\left(\bar{\theta}_{w}\right)^{-1} I_{\mathfrak{w}}^{-1}(\mathcal{U})$ is open in $Z_{w}$ and hence $I_{\mathfrak{w}}^{-1}(\mathcal{U})$ is open in $X_{\mathfrak{w} \text {,rep }}^{o}$. To prove that $I_{\mathfrak{w}}$ is a morphism, it suffices to show that for any affine open $\mathcal{U} \subset \hat{X}_{n}^{o}$, the map $I_{\mathfrak{w}_{I_{\mathfrak{w}}}^{-1}(\mathcal{U})}: I_{\mathfrak{w}}^{-1}(\mathcal{U}) \rightarrow \mathcal{U}$ is a morphism: But this follows from Proposition (4.1), since the map $I_{\mathfrak{w}} \circ \bar{\theta}_{w}=\theta_{w}$ is a morphism.

Conversely, fix $n \geq 0$ and take $\hat{X}_{n}^{o}$. Then $I^{-1}\left(\hat{X}_{n}^{o}\right) \subset X_{\text {rep }}^{o}$ is a closed subset and moreover (by Lemma C.10), $I^{-1}\left(\hat{X}_{n}^{o}\right) \subset X_{m}^{o}$ (for some $m$ ), in particular, $I^{-1}\left(\hat{X}_{n}^{o}\right)$ is a projective subvariety of $X_{\text {rep }}^{o}$. The bijective map $I_{n}: I^{-1}\left(\hat{X}_{n}^{o}\right) \rightarrow \hat{X}_{n \text {, lat }}^{o}$ (where $\left.I_{n}:=I_{I_{I}-1\left(\hat{X}_{n}^{o}\right)}\right)$ is a morphism (since $X_{m}^{o} \subset X_{\mathfrak{w}}^{o}$, for some $\mathfrak{w} \in \widetilde{W} / W$ ). Further, the variety $\hat{X}_{n, \text { lat }}^{o}$ is isomorphic with the variety $\operatorname{Gr}(n N, 2 n N)^{1+\bar{t}_{n}}$ (cf. $\S \mathrm{C} .7$ ). But $\operatorname{Gr}(n N, 2 n N)^{1+\bar{t}_{n}}$ is known to be irreducible and normal by using a result of Kostant (cf. $\left[\mathrm{Ku}_{2}\right]$ ). Moreover, $I_{n}$ being a homeomorphism (since $I_{n}$ is a proper surjective morphism), $I^{-1}\left(\hat{X}_{n}^{o}\right)$ is irreducible as well. Hence by [Mum, page 288, I. Original form], $I_{n}$ is an isomorphism. This shows that the identity map $X_{\text {lat }}^{o} \rightarrow X_{\text {rep }}^{o}$ also is a morphism, proving the proposition.

So from now on, we identify $X_{\text {lat }}$ with $X_{\text {rep }}$ and just denote them by $X$. We have the following proposition determining $\operatorname{Pic}(X)$.

(C.13) Proposition. The map $\mathbb{Z} \rightarrow \operatorname{Pic}(X)$ given by

$$
d \mapsto \mathfrak{L}\left(d \chi_{o}\right)
$$

is an isomorphism.

Proof. For any $\mathfrak{w} \in \widetilde{W} / W$, since $X_{\mathfrak{w}}$ is a projective variety, by GAGA, the natural map

$$
\operatorname{Pic}\left(X_{\mathfrak{w}}\right) \stackrel{\sim}{\rightarrow} \operatorname{Pic}_{a n}\left(X_{\mathfrak{w}}\right)
$$

is an isomorphism, where $\operatorname{Pic}_{a n}\left(X_{\mathfrak{w}}\right)$ is the set of isomorphism classes of analytic line bundles on $X_{\mathfrak{w}}$.

We have the sheaf exact sequence:

$$
0 \rightarrow \mathbb{Z} \rightarrow \mathcal{O}_{a n} \rightarrow \mathcal{O}_{a n}^{*} \rightarrow 0
$$

where $\mathcal{O}_{a n}\left(\operatorname{resp} . \mathcal{O}_{a n}^{*}\right.$ ) denotes the sheaf of analytic functions (resp. the sheaf of invertible analytic functions) on $X_{\mathfrak{w}}$. Taking the associated long exact cohomology sequence, we get

$$
\cdots \rightarrow H^{1}\left(X_{\mathfrak{w}}, \mathcal{O}_{a n}\right) \rightarrow H^{1}\left(X_{\mathfrak{w}}, \mathcal{O}_{a n}^{*}\right) \stackrel{c_{1}}{\rightarrow} H^{2}\left(X_{\mathfrak{w}}, \mathbb{Z}\right) \rightarrow H^{2}\left(X_{\mathfrak{w}}, \mathcal{O}_{a n}\right) \rightarrow \ldots
$$

where the map $c_{1}$ associates to any line bundle its first Chern class. Now

$$
H^{i}\left(X_{\mathfrak{w}}, \mathcal{O}\right)=0, \quad \text { for all } i>0
$$

by $\left[\mathrm{Ku}_{1}\right.$, Theorem 2.16(3)] (also proved in [M]); and by GAGA 
and hence the map $c_{1}$ is an isomorphism. But

$$
\operatorname{Pic}_{a n}\left(X_{\mathfrak{w}}\right) \approx H^{1}\left(X_{\mathfrak{w}}, \mathcal{O}_{a n}^{*}\right)
$$

Hence, by combining (1) and (3)-(6), we get the isomorphism (again denoted by)

$$
c_{1}: \operatorname{Pic}\left(X_{\mathfrak{w}}\right) \stackrel{\approx}{\rightarrow} H^{2}\left(X_{\mathfrak{w}}, \mathbb{Z}\right)
$$

Further, the following diagram is commutative (whenever $X_{\mathfrak{w}} \subseteq X_{\mathfrak{v}}$ ) :

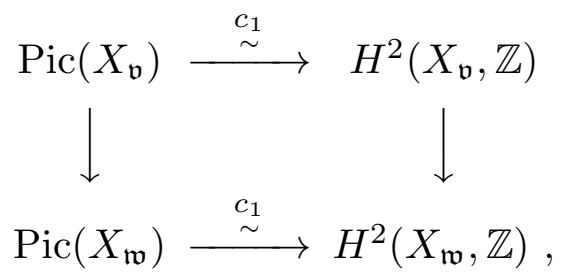

where the vertical maps are the canonical restriction maps. But from the Bruhat decomposition, for any $\mathfrak{w} \geq \mathfrak{s}_{o}$, the restriction map

$$
H^{2}\left(X_{\mathfrak{w}}, \mathbb{Z}\right) \rightarrow H^{2}\left(X_{\mathfrak{s}_{o}}, \mathbb{Z}\right)
$$

is an isomorphism, where $s_{O}$ is the (simple) reflection corresponding to the simple coroot $\alpha_{0}^{\vee}$, and $\mathfrak{s}_{o}:=s_{o} \bmod W$. Moreover, $X_{\mathfrak{s}_{o}}$ being isomorphic with the complex projective space $\mathbb{P}^{1}, H^{2}\left(X_{\mathfrak{s}_{o}}, \mathbb{Z}\right)$ is a free $\mathbb{Z}$-module of rank 1 , which is generated by the first Chern class -1 of the line bundle $\mathfrak{L}\left(\chi_{o}\right)_{\mid X_{\mathfrak{s}_{o}}}$. In particular, $\operatorname{Pic}\left(X_{\mathfrak{w}}\right)$ is freely generated by $\mathfrak{L}\left(\chi_{o}\right){ }_{\mid}$, for any $\mathfrak{w} \geq \mathfrak{s}_{o}$.

We next prove that the canonical map $\alpha$ : Pic $X \rightarrow \varliminf_{\mathfrak{w} \in \widetilde{W} / W} \operatorname{Pic}\left(X_{\mathfrak{w}}\right)$ is an isomorphism:

Since the line bundles $\mathfrak{L}\left(d \chi_{0}\right)$ (for $d \in \mathbb{Z}$ ) are algebraic line bundles on $X$, the surjectivity of the map $\alpha$ follows. Now we come to the injectivity of $\alpha$ :

Let $\mathfrak{L} \in \operatorname{Ker} \alpha$. Fix a non-zero vector $v_{o}$ in the fiber of $\mathfrak{L}$ over the base point $\mathfrak{e} \in X$. Then $\mathfrak{L}_{\mid X_{\mathfrak{w}}}$ being a trivial line bundle on each $X_{\mathfrak{w}}$, we can choose a nowhere-vanishing section $s_{\mathfrak{w}}$ of $\mathfrak{L}_{\mid X_{\mathfrak{w}}}$ such that $s_{\mathfrak{w}}(\mathfrak{e})=v_{o}$. We next show that for any $\mathfrak{v} \geq \mathfrak{w}, s_{\mathfrak{v}_{\mid X_{\mathfrak{w}}}}=s_{\mathfrak{w}}$ : Clearly $s_{\mathfrak{v}_{\mid X_{\mathfrak{w}}}}=f s_{\mathfrak{w}}$, for some regular function $f: X_{\mathfrak{w}} \rightarrow \mathbb{C}^{*}$. But $X_{\mathfrak{w}}$ being projective and irreducible, $f$ is constant and in fact $f \equiv 1$ since $s_{\mathfrak{v}}(\mathfrak{e})=s_{\mathfrak{w}}(\mathfrak{e})$. This gives rise to a nowhere-vanishing (regular) section $s$ of $\mathfrak{L}$ on the whole of $X$ such that $s_{\mid X_{\mathfrak{w}}}=s_{\mathfrak{w}}$. From this it is easy to see that $\mathfrak{L}$ is isomorphic with the trivial line bundle on $X$. This proves that $\alpha$ is injective, thereby completing the proof of the proposition.

\section{REFERENCES}

[AM] Atiyah, M.F., and Macdonald, I.G., Introduction to commutative algebra, Addison-Wesley, 1969.

[B] Borel, A., Linear Algebraic Groups, W.A. Benjamin, New York, 1969.

[BL] Beauville, A., and Laszlo, Y., Conformal blocks and generalized theta functions, Commun. Math. Phys. 164 (1994), 385-419. 
[D] Dynkin, E.B., Semisimple subalgebras and semisimple Lie algebras, American Math. Soc. Translations series 26 (1957), 111-244.

[DN] Drezet, J.M. and Narasimhan, M.S., Groupe de Picard des variétés de modules de fibrés semi-stables sur les courbes algébriques, Inven. Math. 97 (1989), 53-94.

[Fa] Faltings, G., A proof for the Verlinde formula, J. Alg. Geom. 3 (1994), 347-374.

[F] Fulton, W., Introduction to Intersection Theory in Algebraic Geometry, Regional Conference Series in Mathematics number 54, AMS, 1984.

[GK] Guruprasad, K. and Kumar, S., A new geometric invariant associated to the space of flat connections, Compositio Math. 73 (1990), 199-222.

$\left[\mathrm{H}_{1}\right]$ Harder, G., Halbeinfache gruppenschemata über Dedekindringen, Invent. Math. 4 (1967), $165-191$.

$\left[\mathrm{H}_{2}\right]$ Harder, G., Halbeinfache gruppenschemate uber vollstandigen kurven, Invent. Math. 6 (1968), 107-149.

[Ha] Hartshorne, R., Algebraic Geometry, Springer, Berlin-Heidelberg-New York, 1977.

[Hu] Humphreys, J.E., Introduction to Lie Algebras and Representation Theory, Graduate Texts in Mathematics Vol 9, Springer, 1972.

[Hur] Hurtubise, J.C., Holomorphic maps of a Riemann surface into a flag manifold, Preprint (1994).

[IM] Iwahori, N. and Matsumoto, H., On some Bruhat decomposition and the structure of the Hecke rings of $\mathfrak{p}$-adic Chevalley groups, Publ. Math. IHES 25 (1965), 237-280.

[K] Kac, V. G., Infinite Dimensional Lie Algebras, Progr. Math. Vol. 44, Birkhäuser, Boston, 1983.

[KL] Kazhdan, D. and Lusztig, G., Schubert varieties and Poincare duality, Pro. Symp. Pure Math. A.M.S. 36 (1980), 185-203.

$\left[\mathrm{Ku}_{1}\right]$ Kumar, S., Demazure character formula in arbitrary Kac-Moody setting, Invent Math. 89 (1987), 395-423.

$\left[\mathrm{Ku}_{2}\right]$ Kumar, S., Normality of certain Springer fibers (handwritten notes) (1986).

[KN] Kumar, S., and Narasimhan, M.S., Picard group of the moduli spaces of G-bundles, Under preparation.

[KNR] Kumar, S., Narasimhan, M.S., and Ramanathan, A., Infinite Grassmannians and moduli spaces of G-bundles, Math. Annalen 300 (1994), 41-75.

[L] Lang, S., Introduction to Arakelov Theory, Springer-Verlag, 1988.

[Le] Le Potier, J., Fibrès vectorielle sur les courbes algèbriques, Cours de DEA, Université Paris 7, 1991.

[M] Mathieu, O., Formules de caracterès pour les algebrès de Kac-Moody générales, Astérisque 159-160 (1988), 1-267.

[Mi] Milne, J.S., Etale Cohomology, Princeton University Press, Princeton, 1980.

[Mum] Mumford, D., The Red Book of Varieties and Schemes, Springer-Verlag Lecture Notes in Mathematics no. 1358, Berlin-Heidelberg, 1988.

[NR] Narasimhan, M.S. and Ramanan, S., Moduli of vector bundles on a compact Riemann surface, Ann. of Math. 89 (1969), 14-51.

[NRa] Narasimhan, M.S. and Ramadas, T.R., Factorisation of generalised theta functions. I, Invent. Math. 114 (1993), 565-623.

[NS] Narasimhan, M.S. and Seshadri, C.S., Stable and unitary vector bundles on a compact Riemann surface, Ann. of Math. 82 (1965), 540-567.

[PS] Pressley, A. and Segal, G., Loop Groups, Oxford Science Publications, Clarendon Press, Oxford, 1986.

[Q] Quillen, D., Determinants of Cauchy-Riemann operators over a Riemann surface, Functional Analysis and its Applications 19 (1985), 31-34.

$\left[\mathrm{R}_{1}\right]$ Ramanathan, A., Stable principal bundles on a compact Riemann surface- Construction of moduli space, Thesis, University of Bombay, 1976.

$\left[\mathrm{R}_{2}\right]$ Ramanathan, A., Stable principal bundles on a compact Riemann surface, Math. Ann. 213 (1975), 129-152.

$\left[\mathrm{R}_{3}\right]$ Ramanathan, A., Deformations of principal bundles on the projective line, Inven. Math. 71 (1983), 165-191.

[Ra] Raghunathan, M. S., Principal bundles on affine space, C.P. Ramanujam - A Tribute, 
[RR] Ramanan, S. and Ramanathan, A., Some remarks on the instability flag, Tohoku Math. J. 36 (1984), 269-291.

[Sa] Safarevic, I.R., On some infinite-dimensional groups. II, Math. USSR Izvestiza 18 (1982), $185-194$.

[S] Segal, G., The topology of space of rational functions, Acta Math. 143 (1979), 39-72.

[Se 1 Serre, J.P., Espaces fibrés algèbriques, Anneau de Chow et applications, Seminaire Chevalley, 1958.

$\left[\mathrm{Se}_{2}\right]$ Serre, J.P., Cohomologie Galoisienne, Springer-Verlag, Berlin-Newyork, 1965.

[Sl] Slodowy, P., On the geometry of Schubert varieties attached to Kac-Moody Lie algebras, Can. Math. Soc. Conf. Proc. on 'Algebraic Geometry' (Vancouver) 6, 1984, pp. 405-442.

[TUY] Tsuchiya, A., Ueno, K. and Yamada, Y., Conformal field theory on universal family of stable curves with gauge symmetries, Adv. Studies in Pure Math. 19 (1989), 459-565.

[W] Witten, E., Quantum field theory, Grassmannians, and algebraic curves, Comm. in Math. Physics 113 (1988), 529-600.

Deptt. of Mathematics, University of North Carolina, Chapel Hill, N. C. 275993250 , USA. 